\title{
Collective behavior of colloids due to critical Casimir interactions
}

\author{
A. Maciołek ${ }^{1,2,3, \text { * } \text { and S. Dietrich }}$, 3 , † \\ ${ }^{1}$ Institute of Physical Chemistry, Polish Academy of Sciences, \\ Kasprzaka 44/52, PL-01-224 Warsaw, Poland \\ ${ }^{2}$ Max-Planck-Institut für Intelligente Systeme, \\ Heisenbergstraße 3, 70569 Stuttgart, Germany \\ ${ }^{3}$ Institut für Theoretische Physik IV, Universität Stuttgart, \\ Pfaffenwaldring 57, 70569 Stuttgart, Germany
}

(Dated: December 20, 2017)

\begin{abstract}
If colloidal solute particles are suspended in a solvent close to its critical point, they act as cavities in a fluctuating medium and thereby restrict and modify the fluctuation spectrum in a way which depends on their relative configuration. As a result effective, so-called critical Casimir forces (CCFs) emerge between the colloids. The range and the amplitude of CCFs depend sensitively on the temperature and the composition of the solvent as well as on the boundary conditions of the order parameter of the solvent at the particle surfaces. These remarkable, moreover universal features of the CCFs provide the possibility for an active control over the assembly of colloids. This has triggered a recent surge of experimental and theoretical interest in these phenomena. We present an overview of current research activities in this area. Various experiments demonstrate the occurrence of thermally reversible self-assembly or aggregation or even equilibrium phase transitions of colloids in the mixed phase below the lower consolute points of binary solvents. We discuss the status of the theoretical description of these phenomena, in particular the validity of a description in terms of effective, one-component colloidal systems and the necessity of a full treatment of a ternary solvent-colloid mixture. We suggest perspectives on the directions towards which future research in this field might develop.
\end{abstract}

\footnotetext{
* amaciolek@ichf.edu.pl

$\uparrow$ dietrich@is.mpg.de
} 


\section{INTRODUCTION}

Finite-size contributions to the free energy of a spatially confined fluid give rise to an effective force per area acting on the confining surfaces [1]. Fisher and de Gennes [2] made the crucial observation that this fluid-mediated interaction acquires a universal, long-ranged contribution $f_{C}$ if the bulk critical point of the fluid is approached. This is due to critical fluctuations, and hence the notion 'critical Casimir force', in analogy with quantum-mechanical Casimir forces which are due to quantum fluctuations of confined electromagnetic fields [3, 4]. In the case of colloidal suspensions with near-critical suspending fluids (referred to as solvents), the typically micrometer-sized colloidal particles act as cavities inside the critical solvent. At the colloid surfaces these cavities impose boundary conditions for the fluctuating order parameter of the solvent and perturb the order parameter field on the length scale of the bulk correlation length $\xi$. Such modifications of the order parameter and the restrictions of its fluctuation spectrum depend on the spatial configuration of the colloids. Following the argument by Fisher and de Gennes [2], this gives rise to CCFs between the colloids, which is attractive for identical particles and has a range set by the bulk correlation length $\xi$ of the solvent. Since $\xi\left(t=\left(T_{c}^{(s)}-T\right) / T_{c}^{(s)} \rightarrow 0\right) \sim|t|^{-\nu}$, where $\nu$ is a standard bulk critical exponent, this range diverges upon approaching the bulk critical temperature $T_{c}^{(s)}$ of the pure solvent.

The collective behavior of colloids dissolved in the near-critical solvent is determined by the interplay between the CCFs and other interactions acting between the constituents. In general, in colloidal suspensions the dissolved particles interact directly via van der Waals interactions; these are attractive and lead to irreversible aggregation (called coagulation) [5]. In charge-stabilized suspensions, the colloids acquire surface charges due to dissociation of the surface groups in water or due to chemical functionalization of the surface of the particles. This causes the formation of electric double layers around the colloids and results in electrostatic repulsion between them. In sterically-stabilized suspensions, the short polymer chains grafted onto the surface of colloidal particles give rise to a repulsive interaction which is of entropic origin. Additionally, the presence of other smaller solute particles or macromolecular additives such as polymers, surfactants, or micelles [6], induces effective entropic interactions between the colloids, called depletion forces, which are predominantly attractive and short-ranged [7, 8]. If the CCFs between colloidal particles are attractive and 
sufficiently strong to overcome the direct repulsive forces, one may expect the occurrence of a thermodynamically stable colloid-rich liquid or solid phase - even in the absence of any direct attractive interactions. If the resulting attractive potential is sufficiently strong, the condensation transition from a colloid-poor ('gas') to a colloid-rich ('liquid') phase may be preempted (on the characteristic time scales of the observations) by the formation of non-equilibrium aggregates in which the colloidal particle stick together. In general, such aggregates may grow or shrink and their structure varies from loose fractals through gels and glasses to crystals, depending on the packing fraction of the colloidal particles in the aggregates and on the strength of the attraction among the colloidal particles.

Beysens and Estève [9] were the first to study experimentally aggregation phenomena for colloids suspended in binary solvents. These authors studied silica spheres immersed in a water-lutidine mixture by using light scattering. They found the formation of aggregates which sediment upon approaching the bulk coexistence region of demixing from the onephase region of the binary liquid mixture at constant composition of the solvent. Strikingly, the observed aggregation was thermally reversible; moving back the thermodynamic state deeply into the one-phase region the sediments dissolved again. In the decade following this pioneering work, quite a number of further experiments were performed leading to a similar behavior for various binary solvents and a variety of colloids. The structure of the aggregates as well as the the kinetics of aggregation and the reverse process of fragmentation have been investigated. Silica, quartz powder, and polystyrene particles immersed in waterlutidine mixtures were studied in Refs. [10 14], whereas in Refs. [15-19] other solvents were employed; for corresponding reviews see Refs. [20 22]. These experiments, which were performed mostly in the one-phase region of a binary liquid mixture, revealed that reversible aggregation (termed flocculation) is accompanied by a strong adsorption phenomenon in the vicinity of the bulk two-phase coexistence curve. Generically, colloidal particles have a preference for one of the two components of the binary solvent. At the surface of the colloid this preference gives rise to an effective surface field conjugate to the order parameter at the surface and thus leads to an adsorption layer rich in this preferred component. The measurements demonstrated that the temperature - composition $(T, c)$ region in which colloidal aggregation appears is not symmetric about the critical composition $c_{c}$ of the binary solvent. Strong aggregation occurs on that side of the critical composition which is rich in the component not preferred by the colloids. 
Various mechanisms were put forward for strong adsorption giving rise to attraction which in turn could explain the occurrence of (non-equilibrium) flocculation. For example, capillary condensation and/or wetting can occur when particles come close together via diffusion, even far off the critical composition of the solvent. In this case a liquid 'bridge' can form which induces attractive solvation forces 23. Another possibility is that the presence of an adsorption layer around the colloidal particles increases the strength of the direct attractive dispersion interactions. However, in the close vicinity of the bulk critical point of the solvent, in line with the predictions of Fisher and de Gennes, effective attraction induced by critical fluctuations is expected be dominant. In their original paper, Beysens and Estève have identified an "aggregation line" in the temperature-composition phase diagram of the solvent with a prewetting line. However, they have observed that this aggregation line extends to temperatures below the lower demixing critical point of a solvent. However, in general such an extent of prewetting lines has not been found up to now, neither for planar nor for spherical substrates. Actually, positive curvature even shortens prewetting lines [25]. (Regrettably, the wetting behavior of this system in planar geometry has never been investigated.) The authors did not comment on this difference nor did they admit the role of critical fluctuations and Casimir forces for aggregation in the critical region. They have presumed that the aggregation process results from the attractive forces between the colloids due to the presence of the adsorption layer and have referred to de Gennes [24] as the one who had proposed such fluctuation induced interactions at and near the bulk critical point of the solvent, but they have not put their results into the proper context of de Gennes's predictions. According to another interpretation of the experimental findings mentioned above, the observed phenomenon is regarded as a precursor of a bona fide phase transition in the ternary mixture rather then a non-equilibrium flocculation of colloidal particles [1618]. A few theoretical [26] and simulation [27, 28] attempts have been concerned with such an interpretation. The status of knowledge about reversible aggregation of colloids in binary solvents up to the late 1990s has been reviewed by Beysens and Narayanan (see Ref. [21] and references therein). More recently, the scenario of 'bridging' transitions has again been studied theoretically in Refs. [29 32].

In spite of the relevance of aggregation phenomena for the stability of colloidal suspensions, the basic understanding of the collective behavior of colloids dissolved in a near-critical solvent has started to emerge only recently. This progress had to await the advances made 
during the last decade concerning the statistical mechanical theory and computer simulations of CCFs. The accumulated theoretical knowledge of two-body CCFs has triggered also an increase of experimental activities in this field. This renewed interest is driven by application perspectives, in particular concerning the buildup of nanostructured materials of well defined structure by using self-assembly of colloidal particles. In order to achieve a desirable morphology of aggregates, one has to be able to control colloidal self-assembly and to manipulate the particles. The remarkable features of CCFs offer such possibilities. The range and the strength of the CCFs, which depend sensitively on temperature via the bulk correlation length $\xi$, can be tuned reversibly and continuously by moving the thermodynamic state of the solvent around its critical point. The sign of $f_{C}$ can be manipulated as well by suitable surface treatments of the colloids [33 35]. Additional interest is sparked by the potential relevance of CCFs for lipid membranes. These are two-dimensional (2d) liquids consisting of two (or more) components, such as cholesterol and saturated and unsaturated lipids, which can undergo phase separation into two liquid phases, one being rich in the first two components and the other rich in the third [36]. Lipid membranes serve as model systems for cell plasma membranes [37]. Recent experiments suggest that cell membranes are tuned to the miscibility critical point of the $2 d$ Ising model [38, 39] so that CCFs may arise between macromolecules embedded in the membrane [40,42].

Other mechanisms, which - similar to the ones generating the critical Casimir effect also induce solvent-mediated long-ranged interactions, occur inter alia in a chemical sol upon approaching its percolation transition [43], in a binary liquid mixture subjected to a steady temperature gradient due to the concomitant nonequilibrium concentration fluctuations [44, in driven noncohesive granular media due to hydrodynamic fluctuations [45], or if the solvent comprises active matter such as bacteria or self-propelled colloidal particles [46, 47].

Compared with other effective forces between colloid particles or macromolecules, CCFs have two advantages. First, due to the concept of universality for critical phenomena, to a large extent CCFs do not depend on microscopic details of the system. Second, whereas adding depletion agents or ions changes the resulting effective forces de facto irreversibly, the tuning of $f_{C}$ via temperature is fully and easily reversible.

The present article discusses current theoretical and numerical approaches towards the description of the static, equilibrium properties of colloidal suspensions with a near-critical binary solvent. The related experimental body of research is put into the corresponding con- 
text and a number of intriguing possible developments are highlighted. Recent developments concerning colloidal assembly due to CCFs with a focus on the experimental observations is reviewed in Ref. [48].

\section{EFFECTIVE ONE-COMPONENT APPROACH}

A common approach to the statistical mechanics description of colloidal suspensions follows the ideas developed for multi-component molecular liquids, such as ionic solutions, by considering the colloidal particles as 'supramolecules' [6, 49, 50]. Within this approach, the degrees of freedom of the solvent and the ions, in the case of charged-stabilized suspensions, are traced out in order to construct an effective one-component system of colloidal particles interacting via state- and configuration-dependent forces. For most cases, carrying out the integration over microscopic degrees of freedom can be done only approximately, leading to additive pairwise interactions between the colloidal particles (see the corresponding discussion below). For any binary mixture with pair interactions for which the volume integral is finite, a formal expression for an effective Hamiltonian, describing particles of one species only but in the presence of the particles of the other species, has been given in Ref. [51]. This effective Hamiltonian consists of zero-body, one-body, two-body, three-body, and higher-body interactions, which depend on the density of the second species and have to be determined one by one. For additive hard-sphere mixtures with a large size asymmetry, a comparison with direct simulations of true binary mixtures has shown that the pairwise (depletion) potential approximation of the effective Hamiltonian between two large particles accounts remarkably well for the phase equilibria, even in limits for which one might expect that higher-body terms cannot be neglected. This success encourages one to use an effective one-component approach (with the approximation of an additive pairwise potential) to colloids suspended in a near-critical solvent, despite the fact that the CCFs are inherently non additive. The critical Casimir interaction between two colloidal particles depends on the (instantaneous) spatial configuration of all colloids [52 55]. Only for dilute suspensions

or for temperatures sufficiently far away from the bulk critical temperature $T_{c}^{(s)}$ of the pure solvent, such that the range of the critical Casimir interaction between the colloids is much smaller than the mean distance between them, the assumption of pairwise additive CCFs is expected to be reliable. 


\section{A. Effective interactions}

In most of the experimentally studied systems, the solvent is a binary mixture of molecular liquids and the colloidal particles are micro-sized spheres of a radius $R$. For such a sizewise highly asymmetric multi-component system, one can ignore the discrete nature of the solvent and use a simplified pair potential model for the background interaction potential between the colloids, which is present also away from the critical temperature $T_{c}^{(s)}$ of the solvent. Such a model is supposed to capture only the essential features of a stable suspension on the relevant, i.e., mesoscopic, length scale. Besides the van der Waals contribution, which will be discussed below, these features are the hard core repulsion for center-to-center distances $r<2 R$ and a soft repulsive contribution, for which one can employ the Yukawa potential. This leads to the screened Coulomb model of suspensions which are charge-stabilized against flocculation [56-58]:

$$
V_{\text {rep }}(D) /\left(k_{B} T\right)=U_{\text {rep }}(D)=\left(U_{0} /(\kappa D)\right) \exp (-\kappa D), \quad D=r-2 R>0
$$

where $D$ is the surface-to-surface distance and $k_{B}$ is the Boltzmann constant. The range $\kappa^{-1}$ of the repulsion is the Debye screening length $\kappa^{-1}=\sqrt{\epsilon \epsilon_{0} k_{B} T /\left(e^{2} \sum_{i} \rho_{i}\right)}$ (see, e.g., Ref. [59]), where $e$ is the elementary charge, $\epsilon$ the permittivity of the solvent relative to the vaccum, $\epsilon_{0}=8.854 \times 10^{-12} \mathrm{C}^{2} /(\mathrm{Jm})$ is the vacuum permittivity, and $\left\{\rho_{i}\right\}$ the number densities of all ions (regardless of the sign of their charges). A simplified, purely exponential form of the repulsive pair potential,

$$
U_{\text {rep }}(D)=A \exp (-\kappa D)
$$

is often used for suspensions in which $\kappa^{-1} \ll R$ for distances $2 R>D>R+\kappa^{-1}$, for which all curvature effects associated with the spherical geometry of the colloidal particles effectively drop out [60, 61]. The corresponding condition $\kappa^{-1} \ll R$ is practically satisfied for the experimentally relevant systems for which the Debye length is of the order of $10 \mathrm{~nm}$ and the colloidal size of the order of $1 \mu \mathrm{m}$. For the effective Coulomb interaction screened by counterions the amplitude $A$ is given by [56]

$$
A=2 \pi\left(\epsilon \epsilon_{0}\right)^{-1} \Upsilon^{2} \kappa^{-2} R /\left(k_{B} T\right)
$$

where $\Upsilon$ is the surface charge density of the colloid. The purely exponential form of repulsion (Eq. (2)) can also describe sterically stabilized suspensions beyond the hard-sphere model 
[60]. In that case the range $\kappa^{-1}$ of the repulsion is associated with the length of the grafted polymers and the strength $A$ of the repulsion depends on the surface coverage of grafted polymers.

Upon approaching the bulk critical point $\left(T_{c}^{(s)}, c_{c}\right)$ of the solvent, CCFs between the particles emerge and the corresponding pair potential $V_{C} /\left(k_{B} T\right)=U_{C}$ adds to the background contribution. In the well defined scaling limit of all length scales of the system being large on molecular scales, $U_{C}$ attains a scaling form [62 64 in terms of suitable dimensionless scaling variables describing the distance between the colloids, the dependence on the thermodynamic state of the solvent, and the shape of the colloidal particles. For example, for the spherical particles one has

$$
\frac{V_{C}(D)}{k_{B} T}=U_{C}(D) \simeq \frac{R}{D} \Theta\left(\mathcal{Y}=\operatorname{sgn}(t) \frac{D}{\xi_{t}}, \Delta=\frac{D}{R}, \Lambda=\operatorname{sgn}\left(h_{b}\right) \frac{D}{\xi_{h}}\right), \quad D=r-2 R>0 .
$$

In Eq. (4), $\Theta$ is a universal scaling function. Here $\xi_{t}(t \gtrless 0)=\xi_{t, \pm}^{(0)}|t|^{-\nu}$, with $t= \pm(T-$ $\left.T_{c}^{(s)}\right) / T_{c}^{(s)}$ for an upper $(+)$ and a lower $(-)$ critical point, respectively, is the true correlation length governing the exponential decay of the solvent bulk two-point order parameter (OP) correlation function for $t \rightarrow 0^{ \pm}$and $h_{b}=0$ where $h_{b}$ is the bulk ordering field conjugate to the OP. The amplitudes $\xi_{t, \pm}^{(0)}$ (with \pm referring to the sign of $t$ ) are non-universal but their ratio $\xi_{t,+}^{(0)} / \xi_{t,-}^{(0)}$ is universal. The correlation length $\xi_{h}=\xi_{h}^{(0)}\left|h_{b}\right|^{-\nu /(\beta \delta)}$ governs the exponential decay of the solvent bulk two-point OP correlation function for $t=0$ and $h_{b} \rightarrow 0$, where $\xi_{h}^{(0)}$ is a non-universal amplitude related to $\xi_{t, \pm}^{(0)}$ via universal amplitude ratios; $\nu, \beta$, and $\delta$ are standard bulk critical exponents [65]. For the demixing phase transition of a binary liquid mixture, the OP $\phi$ is proportional to the deviation of the concentration of species, say $a$,

$$
c_{a}=\varrho_{a} /\left(\varrho_{a}+\varrho_{b}\right)
$$

from its value $c_{a, c}$ at the critical point, i.e., $\phi \sim c_{a}-c_{a, c}$; here $\varrho_{\alpha}, \alpha \in\{a, b\}$, are the number densities of the particles of species $a$ and $b$, respectively. The bulk ordering field, conjugate to this order parameter, is proportional to the deviation of the difference $\Delta \mu=\mu_{a}-\mu_{b}$ of the chemical potentials $\mu_{\alpha}, \alpha \in\{a, b\}$, of the two species from its critical value, i.e., $h_{b} \sim \Delta \mu-\Delta \mu_{c}$. We note, that the actual scaling fields of fluids are linear combinations of $h_{b}$ and of the reduced temperature $t$.

The minimal model for a pair potential describing the effects of a critical solvent on dissolved colloids due to CCFs, i.e., corresponding to the sum of Eqs. (2) and (44), has been 
used in Refs. [66 72]. In Ref. [73], instead of a soft repulsive potential of the form as in Eqs. (2) and (3), the electrostatic repulsion has been modeled via a hard disc repulsion with an effective diameter. In some of the studies cited above, a simplified functional form of the universal scaling function $\Theta$ has been employed [68, 69, 71, 72], such as using a form valid only asymptotically for large values of the temperature scaling variable neglecting the dependence on the other, also relevant scaling variables. Since, however, the shape of the total pair potential depends sensitively on details of the CCFs pair potential, there is a need to discuss them (see Subsec. II B below).

In order to be able to describe certain experimental systems, one has to consider also the interaction which accounts for effectively attractive dispersion forces. For two spheres, the (nonretarded) van der Waals forces contribute to the total potential through a term (see, e.g., Refs. [59, 74])

$$
V_{\mathrm{vdW}}(\Delta) /\left(k_{B} T\right)=U_{\mathrm{vdW}}(\Delta)=-\frac{A_{H} /\left(k_{B} T\right)}{6}\left[\frac{2}{\Delta(\Delta+4)}+\frac{2}{(2+\Delta)^{2}}+\ln \left(\frac{1+4 / \Delta}{(1+2 / \Delta)^{2}}\right)\right]
$$

where $A_{H}$ is the Hamaker constant. As $\Delta=D / R$ increases, this term crosses over from the behavior $U_{\mathrm{vdW}}(D \ll R) \simeq-\left(A_{H} / 6\right)(R / D)^{2}$ to $U_{\mathrm{vdW}}(D \gg R) \simeq-\left(2 A_{H} / 3\right)(R / D)^{3}$. The Hamakar constant depends on the dielectric properties of the materials involved in the experiment under consideration [59]. By using index-of-refraction-matched colloidal suspensions [60] its value can be strongly reduced. This way dispersion forces can be effectively switched off. (A detailed discussion of the Hamakar constant for a polystyrene colloid near a silica glass substrate immersed in a mixture of water and 2,6-lutidine can be found in Ref. [34.)

Additionally, in the presence of small co-solutes such as free polymer coils or smaller colloids in a stericly stabilized colloidal suspension, one has to consider depletion interactions which arise between large colloidal particles due to entropic effects caused by the small solutes. This so-called depletion interaction is mainly attractive and has a range proportional to the size of the depletant [7, 8]. There are several theories and approximations for the depletion potential, which are summarized in Refs. [75, 76]. For example, for a fluid of large hard spheres of radius $R$ and a small spherical depletant of diameter $\sigma$, which on its own 
behaves as an ideal gas, the depletion potential is [8]

$$
V_{d}(D) /\left(k_{B} T\right)=U_{d}(D)= \begin{cases}-n_{b} V_{o v}(D), & 0 \leq D \leq \sigma \\ 0, & D \geq \sigma,\end{cases}
$$

where $V_{o v}(D)=(\pi / 6)(\sigma-D)^{2}(3 R+\sigma+D / 2)$. In Eq. (7) $n_{b}$ is the bulk number density of the small spheres. Note that $\left|V_{d}(D)\right|$ in Eq. (7) is equal to the pressure $\left(p=n_{b} k_{B} T\right)$ times the overlap volume $V_{\text {ov }}$ between the excluded volumes denied to the centers of the small spheres around each big sphere. Taking into account hard-core repulsion among the depletants produces a repulsive contribution to the depletion interaction as well as an oscillatory decay at large distances [75, 76]. For many actual colloidal suspensions the depletion attraction is strong enough to induce colloidal aggregation and, despite of its rather short range, a gasliquid-like phase separation, because even a small degree of polydispersity or non-sphericity of the particles causes the fluid phase to be not preempted by crystallization.

If the depletant is only a co-solute in the suspension in which the solvent becomes critical, the resulting depletion potential simply adds to the background potential. It may, however, occur that due to an effective depletant-depletant interaction the depletant itself exhibits a phase transition with a critical point. This means that the solvent, which is common to both the big spheres and the depletant, does not display a critical point of its own as the one discussed above. A system which realizes this interesting scenario was studied experimentally in Refs. [77, 78] and via Monte Carlo simulations [79] (see Secs. II B 5 and IV E below where we shall review the results of these studies). In such a case one expects that the depletant produces one unique effective pair potential between the big particles. Far away from the critical point of the depletant, this unique pair potential has the character of a depletion interaction, whereas close to the critical region of the depletant it should display the features of the CCFs pair potential. As has been shown in Refs. [77, 78], the framework of density functional theory (DFT) [80], which is commonly adopted in colloidal science, can provide both forms for the effective pair potential in the corresponding limits (see Sec. II B below for details). In Ref. [79], the effective pair potential $V_{\text {eff }}(r)$ between two big hard-sphere colloids has been determined numerically for two models of the depletant particles within a wide range of state points, including the critical region. In the first model, the spherical 
depletant particles interact via a pairwise square-well potential (SW):

$$
V^{S W}(r)= \begin{cases}\infty, & r<\sigma \\ -d_{w}, & \sigma \leq r \leq\left(1+r_{w} \sigma\right) \\ 0, & r \geq\left(1+r_{w}\right) \sigma\end{cases}
$$

where $r$ is the center-to-center distance between two depletant particles, $r_{w}$ is a dimensionless well width, and $d_{w}$ is the well depth. The second depletant model is an anisotropic threepatches (3P) Kern-Frenkel system which consists of hard-sphere particles decorated with three attractive sites [81]. The critical packing fraction of these patchy particles is very small - as in the experimental system studied in Refs. [77, 78]. For this kind of depletant, the numerically determined effective pair potential $V_{\text {eff }}(r)$ between two big spheres has subsequently been used in a grand canonical off-lattice MC simulation in order to analyze the stability of the colloidal suspension for a system of colloidal particles interacting via the pairwise additive interaction $V_{H S}(r)+V_{\text {eff }}(r)$, where $V_{H S}(r)$ is a hard-sphere potential. The results of this study will be discussed below in Sec. IIB 5.

\section{B. Critical Casimir pair potential}

A crucial ingredient for an effective one-component approach is to have an accurate critical Casimir potential (CCP) between two colloidal particles. This is not only relevant for the investigation of aggregation or the bulk phase behavior of colloids, but it is also

of intrinsic scientific interest. In recent years an experimental technique (total internal reflection microscopy (TIRM) [82 84]) has been developed which allows one to measure directly and with fN resolution the effective potential of the CCF between a colloidal particle, suspended in a near-critical binary mixture, and a fixed object such as a planar wall [33-35]. Video microscopy has also been used in order to determine the potential of the CCF between two spherical colloids [71, 72, 85, 86]. Although the resolution and the sophistication of such experiments increase, it is difficult to interpret the data of these measurements mainly due to the inevitable, simultaneous presence of various contributions to the effective pair potential. Therefore reliable theoretical results are required in order to improve the interpretations.

The solvent-mediated force between two spherical particles, a surface-to-surface distance 
$D$ apart, is defined as the negative derivative of the excess free energy $\mathcal{F}^{e x}=\mathcal{F}-V f_{b}$,

$$
f_{s}=-\frac{\partial \mathcal{F}^{e x}}{\partial D}=-\frac{\partial\left(\mathcal{F}-V f_{b}\right)}{\partial D},
$$

where $f_{b}$ is the bulk free energy density of the solvent and $\mathcal{F}$ is the free energy of the solvent in the macroscopically large volume $V$ excluding the volume of two suspended colloids. The $\mathrm{CCF} f_{C}$ is the long-ranged universal contribution to $f_{s}$ which emerges upon approaching the bulk critical point of the solvent. The associated critical Casimir potential $(\mathrm{CCP})$ is

$$
V_{C}\left(D, R, T, h_{b}\right) \equiv \int_{D}^{\infty} d z f_{C}\left(z, R, T, h_{b}\right)
$$

so that the critical Casimir force $(\mathrm{CCF})$ is given by $f_{C}\left(D, R, T, h_{b}\right)=-\frac{\partial}{\partial D} V_{C}\left(D, R, T, h_{b}\right)$. According to finite-size scaling theory [62, 63], the CCP exhibits scaling described by a universal scaling function $\Theta$ as given by Eq. (4). This scaling function is determined solely by the so-called universality class of the continuous phase transition occurring in the bulk, the geometry of the setup, and the surface universality classes of the confining surfaces 87 90]. The relevant bulk universality class for colloidal suspensions is the Ising universality class in spatial dimension $d=3$ or $d=2$. For the CCF one has

$$
f_{C} /\left(k_{B} T\right) \simeq \frac{R}{D^{2}} \vartheta(\mathcal{Y}, \Delta, \Lambda)=\frac{R}{D^{2}}\left[\Theta-\mathcal{Y} \frac{\partial}{\partial \mathcal{Y}} \Theta-\Delta \frac{\partial}{\partial \Delta} \Theta-\Lambda \frac{\partial}{\partial \Lambda} \Theta\right] .
$$

The main difficulty in determining theoretically the scaling function of CCFs and their potential lies in the character of the critical fluctuations; an adequate treatment has to include non-Gaussian fluctuations. Usually colloidal particles exert a potential on the surrounding fluid which is infinitely repulsive at short distances and attractive at large distances. Such potentials give rise to pronounced peaks in the density profile of the fluid near the surface of the particle, i.e., there is strong adsorption. In terms of a field-theoretical description this corresponds to the presence of a strong (dimensionless) surface field $h_{s} \gg 1$. For binary liquid mixtures one has $h_{s} \sim\left(\delta \Delta \mu_{s}\right) /\left(k_{B} T\right)$ so that there is a local increment at the surface of the chemical potential difference between the two species. It determines which species of the solvent is preferentially adsorbed at the surface of the colloid. The preference for one component of a binary liquid mixture may be so strong as to saturate the surface of the colloids with the preferred component, which corresponds to $h_{s}=+\infty(-\infty)$. For two colloids this gives rise to symmetry-breaking boundary conditions (denoted by $(+,+)$ or $(-,-)$ ) for the solvent order parameter. The resulting spatial variation of the order parameter poses 
a significant complication for obtaining analytic results. Moreover, non-planar geometries lower the symmetry of the problem. Apart from a few exceptions and limiting cases, the presently available analytical results for CCFs are of approximate character.

At first, for two spherical colloidal particles, the corresponding $\mathrm{CCP}$ has been studied theoretically right at the bulk critical point. De Gennes [24] has proposed the singular effective interaction potential between two widely separated spheres by using a free energy functional, which goes beyond mean-field in the sense that it incorporates the non-classical bulk critical exponents, but neglects the critical exponent $\eta ; \eta$ is the standard bulk critical exponent for the two-point correlation function at criticality with $\eta \sim 0.04$ in $d=3$ and $\eta=1 / 4$ in $d=2$ [65]. Within this approach he has found that for $d=3$ the energy of interaction in the limit $D \gg R$ is $-21.3 \times k_{B} T_{c}^{(s)} R / D$. For that interaction potential, the so-called protein limit, which corresponds to $D / R, \xi / D \gg 1$ and which is based on exact arguments using conformal invariance, renders, within a small-sphere expansion [91, 92,

$$
\begin{aligned}
U_{C}\left(D ; T=T_{c}^{(s)}, h_{b}=0, R\right) \sim R^{d-2+\eta} D^{-(d-2+\eta)} \stackrel{d=3}{=}(R / D)^{-(1+\eta)} \\
\stackrel{d=2}{=}(R / D)^{\eta}
\end{aligned}
$$

which implies for the scaling function in Eq. (4) $\Theta(\mathcal{Y}=0, \Delta=D / R \rightarrow \infty, \Lambda=0) \sim$ $\left.\Delta^{-(d-3+\eta}\right) \stackrel{d=3}{=} \Delta^{-\eta}$. In Ref. [91] the amplitude of $U_{C}$ in $d=3$ has been estimated to be slightly larger than $\sqrt{2}$, which is a factor of ca 15 smaller than the above prediction given by de Gennes [24]. In the opposite, the so-called Derjaguin limit $D \ll R$, one has [91, 92 ]

$$
\begin{aligned}
U_{C}\left(D ; T=T_{c}^{(s)}, h_{b}=0, R\right) \sim R^{(d-1) / 2} D^{-(d-1) / 2} \stackrel{\stackrel{d=3}{=}}{=} R D^{-1}=\Delta^{-1} \\
\stackrel{d=2}{=}(R / D)^{1 / 2}=\Delta^{-1 / 2},
\end{aligned}
$$

which in turn implies for the scaling function in Eq. (4) $\Theta\left(\mathcal{Y}=0, \Delta=\frac{D}{R} \rightarrow 0, \Lambda=0\right)=$ const. These results confirm that the CCFs can indeed successfully compete with direct dispersion [93, 94] or electrostatic forces in determining the stability and phase behavior of colloidal systems.

\section{Mean field theory}

Concerning the full range of parameters, theoretical predictions for the universal scaling function of the CCP between spheres are available only within mean field theory [95, 97]. 
Within this Landau - Ginzburg - Wilson approach, the CCF is conveniently calculated using the stress tensor $\mathcal{T}(\phi(\mathbf{r}))$ in terms of the mean field profile $\phi(\mathbf{r})$ [98]:

$$
\mathbf{f}_{C}=k_{B} T \int_{\mathcal{A}} d^{d-1} r \mathcal{T}(\phi(\mathbf{r})) \cdot \mathbf{n}
$$

where $\mathcal{A}$ is an arbitrary $(d-1)$-dimensional surface enclosing a colloid, $\mathbf{n}$ is the outward normal of this surface, and $\mathbf{f}_{C}=f_{C} \mathbf{e}$ is the force between two colloids, where $\mathbf{e}$ is a unit vector along the line connecting their centers. The orientation of $\mathbf{e}$ is such that $f_{C}<0(>0)$ corresponds to attraction (repulsion). In most cases the mean-field profile $\phi(\mathbf{r})$ is determined by numerical minimization of the Landau-Ginzburg-Wilson Hamiltonian encompassing suitable surface contributions from the colloid surfaces in order to account for symmetry-breaking boundary conditions there. In $d=3$ spatial dimensions such a theory is approximate. In the spirit of a systematic expansion in terms of $\epsilon=4-d$, it is exact in $d=4$ for four-dimensional spheres. Recently, the same approach has been employed in order to calculate the scaling function of the CCFs for three-dimensional spheres posing as hypercylinders $\left(H_{d=4, d^{*}=3}\right)$ in spatial dimension $d=4[1]$ [70], where $H_{d, d^{*}}=\left\{\mathbf{r}=\left(\mathbf{r}_{\perp}, \mathbf{r}_{\|}\right) \in \mathbb{R}^{\mathbf{d}^{*}} \times \mathbb{R}^{\mathbf{d}-\mathbf{d}^{*}}|| \mathbf{r}_{\perp} \mid \leq \mathbf{R}\right\}$. The obtained results differ from the ones for four-dimensional spherical particles $H_{d=4, d^{*}=4}$ in $d=4$. This raises the question whether $H_{d=4, d^{*}=3}$ or $H_{d=4, d^{*}=4}$ renders the better meanfield approximation for the physically relevant case of three-dimensional spheres $H_{d=3, d^{*}=3}$ in $d=3$. Due to this uncertainty more accurate theoretical approaches are highly desirable. Recent MC simulations of a sphere near a wall constitute a first step in this direction [99].

The mean field Landau-Ginzburg-Wilson theory is a versatile approach for calculating CCFs. In the case of the simple film geometry, this approximate approach reproduces correctly the qualitative behavior of CCFs (i.e., their sign, functional form, and structure) for various combinations of surface universality classes. Therefore it has been used to calculate CCFs for various shapes of colloidal particles and for other geometries. Non-spherical, i.e., highly ellipsoidal or sphero-cylindrical colloids, or elongated particles such as cylindrical micelles [100], block copolymers [101, the mosaic tobacco virus [102, and carbon nanotubes [103] are experimentally available and widely used in the corresponding current research efforts, with application perspectives towards new materials in mind. The orientation dependent CCP for ellipsoidal particles near a planar wall at vanishing bulk field $h_{b}=0$ has been studied in Ref. [104]. In this case, due to the anisotropy of the particles, there is

\footnotetext{
${ }^{1}$ In the present notation $d$ and $d^{*}$ correspond to $D$ and $d$ used in Ref. [70], respectively.
} 
not only a force but also a torque acting on the particle. This may lead to additional interesting effects such as the orientational ordering of nonspherical colloids in a critical solvent. The behavior of hypercylinders $H_{d=4, d^{*}=3}$ and $H_{d=4, d^{*}=2}$ near planar, chemically structured substrates have been studied in Ref. [105]. In Ref. [52], the scaling function associated with CCFs for a system consisting of two spherical particles facing a planar, homogeneous substrate has been calculated. This allows one to determine the change of the lateral CCF between two colloids upon approaching a wall which acts like a large third body. Within the applied mean field theory, this many body contribution can reach up to $25 \%$ of the pure pair interaction. As one would expect, the many-body effects were found to be more pronounced for small distances, as well as for temperatures close to criticality. This trend has been confirmed by studying three parallel hypercylinders within mean field theory [53] and three discs in $d=2$ within MC simulations [54]. Three-body interactions in $d=3$ for

spherical colloids have been determined experimentally [55]. However, at the present stage these data cannot yet be compared quantitatively with theoretical results. In Ref. [106] the CCP for and the ensuing alignment of cylindrical colloids near chemically patterned substrates has been determined within mean field theory. The case in which the particles exhibit spatially inhomogeneous surface properties, forming so-called Janus particles which carry two opposing boundary conditions, has also been considered [107]. The experimental fabrication of such particles is of research interest in itself [107, 108] as is the theoretical understanding of the interactions between spherical [107, 109] or non-spherical Janus particles [107, 110], because they are considered to be promising building-blocks for self-assembling materials [111-113].

\section{Beyond mean field theory}

Beyond mean field theory, theoretical predictions for the CCP for two spheres immersed in a critical fluid are available from conformal field methods, which, however, are restricted to the bulk critical point of the solvent and to $d=2$. As mentioned in passing, the exact analytical results for the limiting behavior of spheres which nearly touch (Eq. (13)) and spheres which are widely separated (Eq. (12) have been obtained by using conformal invariance of the free energy and taking the Derjaguin limit in the first case and applying the small-sphere expansion in the second case, respectively [91]. For the $2 d$ Ising universality 
class, the CCP has been calculated numerically [91] within the full range of distances $D$ between two spheres. More recently this was achieved also analytically [41] via the partition function of the critical Ising model on a cylinder, using a conformal mapping onto an annulus. For non-spherical colloidal particles with their dummbell or lens shapes being small compared to the correlation length and to the interparticle distances, exact results for the orientation-dependent CCFs have been obtained by using a small-particle operator expansion and by exploiting conformal invariance for $d \lesssim 4$ and $d=2$ [114]. In fact, in $d=2$ conformal field theory provides a general scheme for critical Casimir interactions between two (or more) objects of arbitrary shape [115]. This can be achieved by using the local conformal mappings of the exterior region of two such objects onto a circular annulus for which the stress tensor is known. Assuming the availability of a simple transformation law for the stress tensor under any such (local) conformal mappings, the CCFs are obtained from the contour integral of the transformed stress tensor along a contour surrounding either one of the two objects [115].

Another nonperturbative approach, which allows one to calculate CCFs directly at a fixed specific spatial dimension - potentially an advantage over field-theoretic approaches based on a systematic expansion in terms of $\epsilon=4-d-$ is the use of semi-empirical free energy functionals for critical inhomogeneous fluids and Ising-like systems. They have been developed by Fisher and Upton [116] in order to extend the original de Gennes-Fisher criticalpoint ansatz [2, 117]. Upon construction, these functionals fulfill the necessary analytic properties as a function of $T$ and a proper scaling behavior for arbitrary $d$. The only input needed is the bulk Helmholtz free energy and the values of the critical exponents. (However, the available functional is valid only for symmetry breaking boundary conditions.) The predictions of this functional for films with $(+,+)$ boundary conditions are in very good agreement with previous results obtained $\epsilon$-expansion and conformal invariance for the scaling function of the order parameter and for the critical Casimir amplitudes [118]. Also the predictions for the full scaling functions of the CCFs at $h_{b}=0$ [119] are in good agreement with results from Monte Carlo simulations. These predictions have been obtained from a linear parametric model, which in the neighborhood of a critical point provides a simple scaled representation of the Helmholtz free energy in terms of "polar" coordinates $(r, \theta)$ centered at the critical point $\left(t, h_{b}, \phi\right)=(0,0,0)$, where $r$ is a measure of the distance from the critical point, and which assumes a linear relationship between the OP $\phi$ and $\theta$. A similar 
local-functional approach proposed by Okamoto and Onuki [120] uses a form of the bulk Helmholtz free energy which differs from the one employed in Ref. [119], in that it is a fieldtheoretic expression for the free energy with (in the sense of renormalization group theory) renormalized coefficients. Such a version does not seem to produce more accurate results for the Casimir amplitudes [120]. Within this renormalized local-functional approach, the CCFs between two spherical particles immersed in a near-critical binary mixture (consisting of $a$ and $b$ particles) has been calculated as a function of both scaling fields, $T$ and $h_{b}$ [31]. The focus of the study is the situation that the $b$-rich phase forms on the colloid surfaces in thermal equilibrium with the subcritical $a$-rich bulk solvent $\left(T<T_{c}^{(s)}\right.$ and $\left.h_{b}<0\right)$. This gives rise to a bridging transition between two spherical particles and the resulting effective forces are of different nature than the CCFs. We note that the validity of the extended de Gennes-Fisher or renormalized local-functional approach in the presence of bulk ordering fields has not yet been tested, not even for the simple film geometry.

\section{Computer simulations}

In cases in which one cannot obtain analytical results, Monte Carlo simulations offer a highly welcome tool in order to overcome the shortcomings of approximate theoretical approaches and to study CCPs within the whole temperature range and also in the presence of the bulk ordering field $h_{b}$. In the case that the solvent is a simple fluid and in the spirit of the universality concept of critical phenomena, one can study the simplest representative of the corresponding universality class of the critical solvent, e.g., spin models such as the Ising lattice gas model. In the case of a lattice, the derivative in Eq. (9) is replaced by a finite difference $\Delta \mathcal{F}^{e x}$ associated with a single lattice spacing. This also requires to introduce a lattice version of a spherical particle. In general, Monte Carlo methods are not efficient to determine quantities, such as the free energy, which cannot be expressed in terms of ensemble averages. Nevertheless, free energy differences can be cast into such a form via, e.g., the socalled "coupling parameter approach" (see, e.g., Ref. [121]). This approach can be applied for systems characterized by two distinct Hamiltonians $\mathcal{H}_{0}$ and $\mathcal{H}_{1}$ but the same configurational space. In such a case, one can introduce the crossover Hamiltonian $\mathcal{H}_{c r}(\lambda)=(1-\lambda) \mathcal{H}_{0}+\lambda \mathcal{H}_{1}$, which interpolates between $\mathcal{H}_{0}$ and $\mathcal{H}_{1}$ as the crossover parameter $\lambda \in[0,1]$ increases from 0 to 1 . The difference between the free energies of two systems characterized by these 
different Hamiltonians can be conveniently expressed as an integral with respect to $\lambda$ over canonical ensemble averages of $\mathcal{H}_{1}-\mathcal{H}_{0}$ (with the averages taken by using the corresponding crossover Hamiltonian for a given value of the coupling parameter). Alternatively, the free energy difference can be determined by integrating the corresponding difference of internal energies over the inverse temperature. The drawback of both methods is that they usually require knowledge of the corresponding bulk free energy density (as in the case of CCFs for a slab geometry). The accurate computation of the bulk free energy density poses a numerical challenge by itself and extracting it from finite-size data requires a very accurate analysis. Moreover, the internal energy differences, when determined by using standard MC algorithms, are affected by huge variances, especially for non-planar geometries such as the sphere - planar wall or the sphere - sphere geometry. In such geometries, the differences between local energies of the two systems with different (by one lattice spacing) sphere - wall or sphere - sphere distances are large only close to the sphere or the wall. This implies that the variance is dominated by the remaining part of the system, for which the local energy difference is very small. Recently a more sophisticated algorithm [99] and new approaches 122 have been developed in order to overcome these problems. The numerical method proposed in Ref. [122] is analogous to the experimental one used by Hertlein et al. [33] according to which the CCP is inferred directly from the Boltzmann distribution function of the positions of the two interacting objects. (In this experiment a sphere performs Brownian motion near a planar substrate.) So far, the improved algorithm of Ref. [99] and the dynamic method of Ref. [122] have been applied only to the slab geometry and to the geometry of a single sphere (or disc) near a planar wall. The only available simulation data for two quasi-spheres in the $d=3$ Ising model have been obtained by using a method based on the integration of the local magnetization over the applied local magnetic field [123]. In that study, the $\mathrm{CCP}$ has been calculated at fixed distances between the two spheres as a function of the temperature scaling variable (related to $\operatorname{sgn}(t)\left(D / \xi_{t}\right)$ ) for a few values of $h_{b}$, or as a function of the bulk field scaling variable (related to $\operatorname{sgn}\left(h_{b}\right)\left(D / \xi_{h}\right)$ ) for several temperatures. Results of this calculation have been obtained only for small separations $D \leq 2 R$, where $R=3.5$ (in units of the lattice spacing $a$ ) is the radius of the particle, because the strength of the potential decreases rapidly upon increasing $D$. The accuracy of these data deteriorates at low temperatures.

Alternative ways of computing the sphere - sphere CCP via MC simulations have been 
used for two-dimensional lattice models. One of them [41] uses Bennet's method [124], according to which one can efficiently estimate the free energy difference between two canonical ensembles, characterized by two different energies $E_{0}$ and $E_{1}$ with the same configuration space, provided that these two ensembles exhibit a significant overlap of common configurations. This method has been employed for the $2 d$ Ising model, with frozen spins forming two quasi-spheres with an effective radius $R$; in the reference ensemble (with energy $E_{0}$ ) the quasi-spheres are separated by a distance $D$ whereas in the second ensemble (with energy $E_{1}$ ), they are separated by a distance $D+a$ [41]. In order to estimate the corresponding free energy difference, within this approach one considers a trial move which keeps the configuration space the same but switches the energy from $E_{0}$ to $E_{1}$, e.g., by mimicking the move of one of the particles from a distance $D$ to a distance $D+a$ by suitably changing and exchanging spins. The estimate of the free energy difference is given by $F_{1}-F_{0}=-\left(1 / k_{B} T\right) \ln \left\langle\exp \left(E_{0}-E_{1}\right) /\left(k_{B} T\right)\right\rangle_{0}$, where the canonical ensemble average $\langle\cdot\rangle_{0}$ is taken with respect to the "reference" ensemble with the energy $E_{0}$. Integrating this free energy difference up to infinity, one obtains the CCP. Reference [41] provides the results for the CCP as a function of distance $D / R \gtrsim 25$ for four values of the temperature in the disordered phase of the solvent, i.e., $T \geq T_{c}^{(s)}$ and $h_{b}=0$. At the critical temperature, these MC simulation results agree well with the analytical ones obtained from conformal field theory. Still another route towards determining the CCP from MC simulations has been followed in Refs. [125, 126] for two quasi-discs immersed in a $2 d$ lattice model of a binary liquid mixture [127]. Here, the pair potential $V_{C}(x, y)$ has been obtained from the numerically determined probability $P(x, y)$ of finding one colloid at position $(x, y)$ provided that another one is fixed at the origin: $V_{C}(x, y)=-k_{B} T \ln (P(x, y) / P(\infty, \infty))$. In order to determine $P(x, y)$ accurately at fixed values of $h_{b} \sim \Delta \mu-\Delta \mu_{c}$ and $T$, the so-called Transition Matrix Monte Carlo technique [128 has been employed. This technique relies on monitoring the attempted transitions between macrostates, as defined, for example, by specific positions of the two quasi-discs, and on using this knowledge in order to infer their relative probability $P(Y) / P\left(Y^{\prime}\right)$, where $Y$ and $Y^{\prime}$ are two distinct states of the system; once sufficient transition data are collected, the entire probability distribution $P(Y)$ can be constructed. The studies in Refs. [125, 126] have been focused on the case that the b-rich phase adsorbs on the colloid surfaces in thermal equilibrium with a supercritical a-rich bulk solvent $\left(T \geq T_{c}^{(s)}\right.$ and $\left.h_{b} \leq 0\right)$. It has been found that data for $U_{C}=V_{C} /\left(k_{B} T\right)$ obtained for $h_{b}=0$ and $D \ll R$ 
collapse on a common master curve if multiplied by $(D / R)^{1 / 2}$ and plotted as a function of $D / \xi_{t}$, i.e., $(D / R)^{1 / 2} U_{C}=\Theta\left(\mathcal{Y}=\operatorname{sgn}(t) D / \xi_{t}, \Delta=D / R=0, \Lambda=\operatorname{sgn}\left(h_{b}\right) D / \xi_{h}=0\right)$. The scaling exponent $1 / 2$ for the prefactor agrees with the prediction in Eq. (13) for $d=2$, which is obtained based on the conformal invariance in the Derjaguin limit $D \ll R$, and which implies $\Theta(y \rightarrow 0, \Delta \rightarrow 0, \Lambda \rightarrow 0)=$ const.

The standard algorithms used for the MC simulation studies described above are based on trial moves generating a trial configuration, which are local (Metropolis-type flips of spins) and hence become very slow near the critical point. This critical slowing down effect can be weakened by using so-called cluster algorithms such as the Swendsen and Wang [129] or the Wolff algorithm [130] in which instead of a single spin a whole cluster of spins is flipped simultaneously. The Wolff algorithm constructs clusters which consist of spins that are aligned and connected by bonds. The proof that the Wolff algorithm obeys detailed balance, and hence generates the Boltzmann distribution, hinges on the spin inversion symmetry of the Hamiltonian. (The Wolff algorithm can be generalized to systems which contain bulk or surface fields [131].) One can exploit also other symmetries in order to to develop a cluster method, for example, by using geometric operations on the spin positions such as, e.g., point reflection or rotation with respect to a randomly chosen "pivot". Hobrecht and Hucht [54] have extended the geometric cluster algorithm (GCA), introduced by Heringa and Blöte for bulk Ising models in the absence of external fields [132], to the case of Ising systems containing areas of spins with a fixed orientation, facilitated by infinitely strong bonds, which mimic a colloidal suspension. The GCA makes use of the invariance of the Hamiltonian with respect to a point inflection in order to construct two symmetric clusters of spins which are then exchanged. Contrary to the Wolff cluster algorithm, the GCA conserves the order parameter. The modification due to Hobrecht and Hucht consists of including into the clusters not only spins but also the bond configuration between them. This way, the particles - encoded into the bond configuration - can be moved and the configuration of a solvent represented by the Ising spins can be updated within one cluster step. In the cluster exchange the neighboring lattice sites $i$ and $j$ as well as the connecting bond $\langle i j\rangle$ are mapped via point reflection with respect to a pivot onto the sites $i^{\prime}$ and $j^{\prime}$ and the bond $\left\langle i^{\prime} j^{\prime}\right\rangle$, respectively. Using this apparently very efficient MC cluster algorithm, the authors of Ref. [54] have studied two-dimensional systems with a fixed number of identical, disclike particles defined as regions of fixed positive spins, which thus effectively impose 
symmetry-breaking $(+)$ BCs onto the surrounding free spins. Within this scheme, they have calculated the two- and three-body CCP at the bulk critical point of the Ising model. The authors report strong finite-size effects: for periodic simulation boxes with a fluctuating total magnetization, the presence of a nonzero number density of colloidal particles with a non-neutral surface preference for up and down spins shifts the system away from the critical point. As a result, their MC results for the CCP do not exhibit the form expected to hold at the critical point for a single pair of particles in solution (Eqs. (12) and (13). In order to suppress this effect, the authors of Ref. [54] have proposed to use a fixed total magnetization $M=0$ or to insert in addition the same number of particles but with the opposite surface preference.

\section{Derjaguin approximation}

Within the so-called Derjaguin approximation curved, smooth surfaces are approximated by surfaces which are a steplike sequence of parallel planar pieces [133]. Between two visà-vis, flat pieces of the opposing surfaces partitioned this way, locally a force acts like in the slab geometry and the total force is taken to be the sum of the forces between each individual pair with the appropriate areal weight. The Derjaguin approximation is widely used to estimate CCFs forces between colloidal particles, because the forces between parallel surfaces are much easier to calculate. Indeed, for the slab geometry the CCFs are known even beyond mean field theory (see below). Therefore, by using this approximation one can account even for non-Gaussian critical fluctuations, albeit at the expense of not fully considering the shape of the particles. The Derjaguin approximation is valid for temperatures which correspond to $\xi_{t} \lesssim R$, because under this condition the CCFs between the colloids act only at surface-to-surface distances $D$ which are small compared with $R$. In many cases this approximation is surprisingly reliable even for $D \lesssim R$ [34, 105]. In the absence of a bulk ordering field, i.e., for $h_{b}=0$ and strongly adsorbing $(+,+)$ confining surfaces the results for the critical Casimir interactions in the film geometry have been provided by field-theoretical studies [134], the extended de Gennes - Fisher local functional method [70, 119, 120], and Monte Carlo simulations of the Ising model [135 137] or improved models which offer the benefit that the amplitude of the leading bulk correction to scaling vanishes [137 140]. Within MC simulations also the case of weakly adsorbing surfaces has been 
considered [141, 142]. In this latter case the corresponding surface field $h_{s}$ might be so small that upon approaching the critical point one effectively observes a crossover of the type of boundary condition imposed on the order parameter from symmetry preserving to symmetry breaking boundary conditions. In this case there appears to be no effective enhancement of the order parameter upon approaching the confining wall. The CCP reflects such crossover behaviors; depending on the film thickness, the CCP can even change sign [141-143]. On the basis of scaling arguments one expects that for moderate adsorption preferences the scaling function of the CCP in film geometry additionally depends on the dimensionless scaling variables $y_{s}^{(i)}=a_{i} h_{s}^{(i)} L^{\Delta_{1} / \nu}, i=1,2$, where $h_{s}^{(1)}$ and $h_{s}^{(2)}$ are the effective surface fields at the two confining surfaces, $a_{1}$ and $a_{2}$ are nonuniversal amplitudes, $\Delta_{1}=0.45672(5)$ [142] is the surface crossover exponent at the so-called ordinary surface phase transition.

The knowledge of the dependence of CCPs on the bulk ordering field $h_{b}$ is rather limited, although it is crucial for understanding the aggregation of colloids near the bulk critical point of their solvent. The presently available MC simulations for Ising films provide such results only along the critical isotherm $T=T_{c}^{(s)}$ [144]. In Refs. [66, 67, 70, 97] the variation of CCFs with $h_{b}$ has been approximated by adopting the functional form obtained within mean field theory (MFT, $d=4$ ) by using a field-theoretical approach within the framework of the Landau - Ginzburg theory, but by keeping the actual critical exponents in $d=3$ for the scaling variables. Within this "dimensional" approximation, the scaling function $\vartheta_{\|}^{(d=3)}=f_{C} D^{3} /\left(k_{B} T \mathcal{S}\right)$ of the CCF for the film geometry ( $\left.\|\right)$ with macroscopically large surface area $\mathcal{S}$ of one wall (see the text below Eq. 10) is taken to be $\vartheta_{\|}^{(d=3)}(\mathcal{Y}=$ $\left.\operatorname{sgn}(t) D / \xi_{t}, \Sigma=\Lambda / \mathcal{Y}=\operatorname{sgn}\left(t h_{b}\right) \xi_{t} / \xi_{h}\right) \simeq \vartheta_{\|}^{(d=3)}(\mathcal{Y}, \Sigma=0) \vartheta_{\|}^{(d=4)}(\mathcal{Y}, \Sigma) / \vartheta_{\|}^{(d=4)}(\mathcal{Y}, \Sigma=0)$, where $\vartheta_{\|}^{(d=3)}(\mathcal{Y}, \Sigma=0)$ has been adopted from MC simulation data [135, 136]. (The normalization by $\vartheta_{\|}^{(d=4)}(\mathcal{Y}, \Sigma=0)$ eliminates a nonuniversal prefactor carried by the Landau - Ginzburg expression for the scaling function of CCFs.) In Ref. [70] the scaling functions $\vartheta_{\|}^{(d=3)}$ resulting from the "dimensional" approximation have been compared with those obtained within the extended de Gennes - Fisher local functional. This allows us to compare in Fig. 1 (unpublished) results for the scaling function $\Theta_{\oplus \oplus}^{(d=3, D e r j)}(\mathcal{Y}, \Sigma)$ of the sphere - sphere $\operatorname{CCP}$ (see Eq. (44) within the Derjaguin approximation, i.e., $\Theta_{\oplus \oplus}^{(d=3, \text { Derj })}(\mathcal{Y}, \Sigma) \simeq(D / R) U_{c}=$ $\pi \int_{1}^{\infty} \mathrm{d} x\left(x^{-2}-x^{-3}\right) \vartheta_{\|}^{(d=3)}(x \mathcal{Y}, \Sigma)$ [70], where $\vartheta_{\|}^{(d=3)}$ is obtained by using these two aforementioned approaches. Only for weak bulk fields the curves in Fig. 1 for both approaches compare well. Otherwise there are clear quantitative discrepancies, the origin of which is 
not clear. The "dimensional" approximation might deteriorate upon increasing $h_{b}$. On the other hand, as already mentioned in passing, the reliability of the local functional approach for $h_{b} \neq 0$ has not yet been tested systematically. For example, the result of the local functional approach for the scaling function of the CCFs along the critical isotherm differs substantially from the one obtained from MC simulations [70]. In Refs. [77, 78] results of a long wavelength analysis of density functional theory for the scaling function of CCF have been reported (see also Sec. II B 5 below ); they are in qualitative agreement with the results obtained using the extended de Gennes - Fisher local functional [70]. The first microscopic off-lattice results for the scaling function of $\mathrm{CCF}$, both along the critical isochore and in the off-critical regime, have been worked out in Ref. [145]. The authors of Ref. [145] have studied a hard core Yukawa model of a fluid by using density functional theory (DFT) within a specific weighted density approximation [146], coupled with hierarchical reference theory [149]. This kind of DFT weighted density approximation, related to the one proposed early on by Tarazona and, independently, by Curtin and Ashcroft [147, 148], captures the short-ranged correlations of the underlying hard sphere fluid rather accurately, whereas the hierarchical reference theory is able to account also for the critical properties of a homogeneous fluid. In Ref. [145], this technique has been applied in order to determine the effective interaction between two hard walls immersed in that fluid. This approach facilitates to investigate the crossover between depletion-like roots of such effective forces at high temperatures and the critical Casimir effect upon approaching the critical point of the fluid. It appears that for hard core Yukawa fluids the universal features of CCF emerge only in close neighborhood of the critical point. The predictions obtained in Ref. [145] for the scaling function of the CCF for various temperatures along the critical isochore $\left(h_{b}=0\right)$ differ significantly from the Ising model MC simulation results in Ref. [136]. Moreover, the data along various isotherms do not collapse as expected from scaling theory. The authors of Ref. [145] interpret these deviations as an indication of strong corrections to scaling occurring in the hard core Yukawa fluid.

As far as experimental data for the film geometry are concerned, they are available only indirectly from measurements of the thickness of wetting films; they correspond to opposing $(+,-)$ boundary conditions [150, 151]. The scaling function of the CCF determined by Fukuto et al. [150] compare well with the theoretical predictions. The measurements performed by Rafai et al. [151] have provided data which show stronger deviations from the 
theoretical curves, in particular around the maximum of the CCF. The scaling function of CCFs for wetting films corresponding to $(+,+)$ boundary conditions could not be determined due to the collapse of the incomplete wetting film in the course of the measurements [151]. Based on exact results in $d=2$ [152 154] and in $d=4$ [134], and based on the predictions from the local-functional approach in $d=3$ [119], it is expected that the $\mathrm{CCF} f_{C}(D)$ between two planar walls with symmetry-breaking boundary conditions at separation $D$ varies as $\exp \left(-D / \xi_{t}\right)$ for $D \gg \xi_{t}$ and $t>0$, i.e., in the one-phase region of the solvent. This implies that the asymptotic behavior of the scaling function is given by $\vartheta_{\|}^{(d=3)}\left(\mathcal{Y}=\operatorname{sgn}(t) D / \xi_{t} \gg\right.$ $\left.1, \Sigma=\operatorname{sgn}\left(t h_{b}\right) \xi_{t} / \xi_{h}=0\right)=\mathcal{A}_{+} \mathcal{Y}^{d} \exp (-\mathcal{Y})$, where $\mathcal{A}_{+}=1.2-1.5$ is a universal number valid for $(+,+)$ boundary conditions 34 . By applying the Derjaguin approximation to this functional form of the CCFs, one obtains the following asymptotic behavior of the scaling function $\Theta_{\oplus \oplus}^{(d=3, \operatorname{Derj})}(\mathcal{Y}, \Sigma=0)$ for the sphere - sphere CCP :

$$
\Theta_{\oplus \oplus}^{(d=3, D e r j)}(\mathcal{Y} \rightarrow \infty, \Sigma=0)=\pi \mathcal{A}_{+} \mathcal{Y} e^{-\mathcal{Y}}, \quad D \gg \xi_{t}, \quad t>0
$$

so that in this limit $U_{C}^{(d=3, D e r j)}=\pi \mathcal{A}_{+} \frac{R}{\xi_{t}} e^{-D / \xi_{t}}$ (Eq. (4)).

\section{Pair potential in the presence of depletants}

In order to calculate the effective pair potential between two large hard spheres immersed into a fluid of depletants close to their gas-liquid critical point, the authors of Refs. [77, 78. have used density functional theory (DFT), which is a powerful tool for describing equilibrium properties of colloidal suspensions (see the last two paragraphs in Sec. II A). The hard spheres have been analyzed within the Derjaguin approximation, which is inherent in recent approaches to depletion forces acting in hard-sphere mixtures. In this context, the Derjaguin approximation relates the force between the two big objects to the integral of the solvation force $\left.f_{\text {solv }}(D)=-(1 / \mathcal{S})(\partial \Omega / \partial D)_{\mu, T, \mathcal{S}}-p\right)$ of the small particles (i.e., the depletant agents) confined between two parallel planar walls with cross-sectional area $\mathcal{S}$. (The film solvation force per surface area $\mathcal{S}, f_{\text {solv }}$, is an excess pressure over the bulk value $p$ of the confined fluid described by the grand potential $\Omega$.) In the limiting case of hard walls exposed to an ideal gas of depletants, this relation reproduces the well known Asakura-Oosawa result for depletion forces [7]. This scheme can be fruitfully applied also to interacting systems. In fact such a relation is equivalent to the general formula obtained by Derjaguin [133] relating 
the force between two convex bodies to the free energy, in excess of its bulk value, of a fluid confined between planar walls. Within an actual DFT approach this formula holds even for approximations of the excess part of an intrinsic Helmholtz free energy functional of a fluid. The authors of Refs. [77, 78] have employed the square gradient local density approximation for the intrinsic Helmholtz free energy functional entering into DFT, which is valid for spatially slowly varying depletant number densities $\rho$ such that $\nabla \rho / \rho \ll 1 / \xi_{t}$. They have simplified the Derjaguin approximation by replacing the two big spheres by two planar parallel walls. Within the square gradient local density approximation, the effective (solvation) force per area $f_{\text {solv }}$ between two parallel walls is expressed solely in terms of the bulk free energy density $f(\rho)$ of the depletant host fluid and in terms of the value of the equilibrium number density profile $\rho\left(z=z_{\text {mid }}\right)$ of such a fluid at the midpoint $z=z_{\text {mid }}$ between the two walls. The solution of the extremum condition for the free energy functional provides the second equation relating, albeit in an implicit way, $f_{\text {solv }}$ and $\rho\left(z=z_{\text {mid }}\right)$ to the density at the wall $\rho(z=0)$, which in turn depends on the wall-fluid interactions. In order to incorporate the non-Gaussian behavior near the gas-liquid critical point of the depletant, in the spirit of the Fisk - Widom or local-functional approach [116, 120, 155], the authors of Refs. [77, 78] have used the scaling form for the singular part of the bulk free energy density of the fluid $\left(f(\rho)-f\left(\rho_{c}\right)\right) /\left(k_{B} T\right)=a_{11} t^{2-\alpha} \Psi(x)$ with the scaling variable $x=b_{1} \phi t^{-\beta}$, where $\phi=\rho-\rho_{c}$ is the bulk order parameter OP, $a_{11}$ and $b_{1}$ are non-universal, dimensional constants, and $\beta$ and $\alpha$ are bulk critical exponents [65]. In the scaling limit, the general expression for the effective force between two parallel walls can be expressed in terms of $\Psi(x)$, the rescaled midpoint OP $x_{0}=b_{1}\left(\rho\left(z_{\text {mid }}\right)-\rho_{c}\right) t^{-\beta}$, and the universal amplitude ratio $g_{4}^{+}$ [65]. This expression takes the scaling form characteristic of CCFs. By using a parametric expression for $\Psi$ [65] and the critical exponents of $3 d$ Ising universality class, the authors have obtained very good agreement between their analytic theory and the scaling function of CCFs obtained from MC simulations [136] at $h_{b}=0$. This is not surprising because, within the square gradient local-density approximation, DFT reduces to the local-functional approach which proved to capture correctly critical fluctuations [116, 118, 119], at least for $h_{b}=0$.

This theoretical approach has been followed up by MC simulation studies [79] aiming at the computation of the CCP between two hard-sphere colloids suspended in an implicit solvent in the presence of interacting depletant particles. In this off-lattice MC simulations 
of fluctuation induced forces, the effective potential between two hard spheres has been determined upon approaching the gas-liquid critical point of the depletant for two different depletant models, one for SW and one for 3P particles (see Eq. (8) and thereafter). Given the computational limitations, the authors have considered the size ratio $q$ between the hard-sphere depletant and the hard-sphere colloid within the range $0.05<q<0.2$. The resulting effective colloid-colloid force has been evaluated by using canonical Monte Carlo simulations for various fixed values of the depletant concentration in the reservoir. The method consists of performing virtual displacements of each colloid from its fixed position and of computing the probability of encountering at least one collision with a depletant particle. The effective colloid-colloid potential follows from integrating the corresponding force. These MC results show that upon cooling the effective potential between two colloidal particles gradually looses its high-temperature, pure hard-sphere depletion character of exhibiting oscillations and transforms into a completely attractive potential with a progressive and significant increase of its range, signaling the onset of critical Casimir forces. For large distances between the surfaces of the two colloids, the MC data for the CCP fit well to the asymptotic form given by Eq. (15). This numerical study has been extended to the case in which colloids interact with SW depletant particles; this interaction has been continuously modified from hard-core repulsion to strong attraction, thus changing from $(-,-)$ boundary conditions (i.e., preference for the gas phase) to $(+,+)$ boundary conditions (i.e., preference for the liquid phase) [156]. For strong colloid-depletant attraction, the effective colloid-colloid potential exhibits oscillations, as they occur for the high-temperature depletion potential, modulating its exponentially decaying attractive tail. The variation of the colloid-colloid effective potential upon crossing over from $(-,-)$ to $(+,-)$ and from $(+,+)$ to $(+,-)$ boundary conditions has been determined, too. In the asymptotic spatial range these two crossovers are the same. However, in the numerical study of Ref. [156] only the behavior at short distances has been probed, where the effective potential is dominated by non-universal aspects of the solvent-colloid interaction so that the aforementioned pairs of boundary conditions are no longer equivalent. 


\section{Main properties of CCFs}

We close this section by listing the main properties of critical Casimir interactions between two colloidal particles in very dilute suspensions. The results for spheres in $d=3$, obtained from the variety of methods described above, tell that they share the same qualitative features of the CCFs with "spheres" in $d=2$ and $d=4$ as well as with planar walls. This refers to the property that for like boundary conditions CCFs are attractive and it refers to the position of a force maximum along various thermodynamic paths. Around the consolute point of a binary solvent the main features of the CCFs between two planar

walls are summarized in Fig. 2 in terms of the force scaling function $\vartheta_{\|}^{(d=4)}(\mathrm{Y}, \Sigma)$ obtained from Landau theory (see Fig. 1 in Ref. [70]). The main message conveyed by Fig. 2 is the asymmetry of the CCFs around the critical point of the solvent with the maximum strength occurring at $h_{b}<0$. This asymmetry is due to the presence of surface fields, which in films lead to capillary condensation [1], whereas between spherical colloids to bridging [23, 29, 31]. Near these phase transitions, the effective force acting between the confining surfaces is attractive, exhibits (within Landau theory) a cusp, and is very strong: the depth of the corresponding CCPs can reach a few hundred $k_{B} T$. The concomitant strong increase of the absolute value of the force is reflected by its corresponding universal scaling function and extends to the thermodynamic region above the capillary condensation or bridging critical point, even to temperatures $T>T_{c}^{(s)}$.

\section{Stability}

Knowing the effective pair potential for the colloids one can investigate the stability of colloidal suspensions and the aggregation of colloids. These phenomena are related to kinetic processes (see Ref. [56] and references therein), which are based on the diffusion of single particles in the presence of other particles of the same kind, interacting with them via interaction potentials which contain both attractive and repulsive contributions. Aggregation occurs if the attractive interactions of the particles dominate over their thermal kinetic energy, which is responsible for the Brownian motion of the particles. Hydrodynamic interactions may also play a role, e.g., by slowing down the aggregation process for solvents with high viscosity. 
In order to quantify the behavior of interacting particles, which irreversibly stick together once their surfaces touch each other, Fuchs has introduced the concept of a stability ratio [157]. It is defined as the ratio $W=J_{0} / J$ between the Brownian motion induced pair formation rate $J_{0}$ in the absence of other than excluded volume interactions between the particles, and the corresponding formation rate $J$ of particle pairs in the presence of such interactions. $W$ can be calculated by extending Smoluchowski's diffusion equation for the radially symmetric relative motion of two coagulating spherical particles of radius $R$ in order to account for their interaction potential [157]:

$$
W=2 R \int_{2 R}^{\infty} \frac{\exp \{U(r)\}}{r^{2}} \mathrm{~d} r .
$$

The analysis, which leads to this expression, is valid only in the early stages of coagulation before triplets etc. are formed. However, it neither deals with the very beginning of the coagulation process but considers only the steady-state situation, which is established quickly. In the analysis based on Smoluchowski's equation hydrodynamic interactions are neglected. From the definition of $W$ it follows that for hard spheres $W=1$, while for $W>1$ $(W<1)$ the repulsive (attractive) part of the pair potential $\left(k_{B} T\right) U$ dominates. In the case of a potential barrier, i.e., if $U(r) \gg 1$ for a certain range of distances $r>2 R$, which leads to $W>1$, one can expect that on intermediate time scales the suspension will be in a (meta)stable homogeneous state. The cluster formation will set in only on a very large time scale. This time scale is proportional to the ratio between the characteristic times for diffusion of a single particle, $t_{d i f f}$, and for the formation of a pair of particles, $t_{\text {pair }}$ [56]:

$$
t_{d i f f} / t_{\text {pair }}=3 \eta / W
$$

with the packing fraction $\eta=(4 \pi / 3) R^{3} \rho$ where $\rho$ is the number density of the colloidal particles. For a near-critical solvent, the pair potential can be taken as (see Eqs. (2) and (4); $\Sigma=\Lambda / \mathcal{Y})$

$$
U(r)= \begin{cases}\infty, & D<0 \\ U_{\text {rep }}+U_{C}^{(d=3)}=A \exp (-\kappa D)+(1 / \Delta) \Theta^{(d=3)}(\mathcal{Y}, \Delta, \Sigma), & D>0 .\end{cases}
$$

For the mixed phase of the solvent the ratio $W$ has been calculated in Ref. [67. The results of this calculation show that the CCF can lead to a rapid coagulation, setting in within a narrow temperature interval, and that the extent of the coagulation region reflects the fact 
that the CCFs are stronger for compositions of the solvent slightly poor in the component preferred by the colloids. The effective pair potential given by Eq. (18) is applicable only for sufficiently large distances $D \gtrsim \kappa^{-1}$, because it takes into account only the electrostatic repulsive interactions and neglects possible short-ranged contributions to the effective vander-Waals interactions. Furthermore, the CCP attains its universal form as given by the scaling function $\Theta^{(d=3)}$ only in the scaling limit, i.e., for distances $D$ which are sufficiently large compared with the correlation length amplitude $\xi_{t,+}^{(0)} \approx 0.25 \mathrm{~nm}$. Analogously, also $\xi_{t}$ and $\xi_{h}$ must be sufficiently large compared with microscopic scales. From the behavior of the scaling function $\Theta^{(d=3)}$ (discussed in Ref. [67] within the Derjaguin approximation) it follows that along the typical thermodynamic paths realized experimentally, the range of attraction due to the CCFs grows steadily upon increasing the bulk correlation length $\xi_{t}$, but the amplitude of the CCFs is a nonmonotonic function of $\xi_{t}$ with its maximal strength attained for an intermediate value of $\xi_{t}$. Depending on the values of $A$ in Eq. (18) and of $\xi_{t}$, the CCFs compensate the repulsion for all values of $D$ or only within certain ranges of $D$, i.e., a secondary attractive minimum of $U(r)$ can occur at a certain distance $D_{\min }$ (in addition to the primary, global minimum at $D \rightarrow 0^{+}$) while for small distances $D<D_{\min }$ and for $D<0$ the potential maintains a repulsive part. The presence of the repulsive barrier and of the attractive secondary minimum in the effective potential, and thus the occurrence of coagulation, depends on temperature. But also in the case, in which for all values of $\xi_{t}$ a repulsive barrier remains, coagulation can appear, due to a deep secondary minimum. The nonmonotonic dependence of the maximal strength of the CCF on temperature results in a nonmonotonic behavior of the ratio $W$, which is shown in Figs. 2 and 3 of Ref. [67].

In Ref. [67, the analysis of the stability ratio for the diversity of possible shapes of the effective pair potential given in Eq. (18) has been complemented by the analysis of the bulk structure of colloidal suspension. To this end the radial distribution function $g(r)$ has been calculated within the integral equation approach by using the hypernetted-chain and the Percus-Yevick closure [158]. The results for both types of closure are almost the same. (The applicability and reliability of this integral equation approach is discussed in detail in Ref. [159].) For temperatures far away from the critical temperature of the solvent, the colloids behave effectively as hard spheres with an effective diameter $\sigma>2 R$ due to the soft repulsive background contribution $U_{r e p}$. Accordingly, for such values of $\xi_{t}, g(r)$ has the corresponding characteristics of a fluid of hard spheres, such as the rather broad first peak 
for small values of $D$. Due to the emerging attractive CCFs, for increasing $\xi_{t}$ the radial distribution function $g(r)$ is enhanced close to the surfaces of the colloids. This implies an enhanced short-ranged order and that the formation of colloidal dimers is favored. The way in which the shape of the radial distribution function $g(r)$ changes upon increasing the temperature reveals whether the effective potential exhibits a repulsive barrier at small values of $D$ and is attractive throughout large distances or whether an attractive minimum develops at intermediate values of $D$ upon increasing temperature while repulsion remains at small and large values of $D$.

One can address the issue whether a relationship can be established between the onset of aggregation and the behavior of a certain quantity, which is accessible both theoretically and experimentally, such as the second virial coefficient $B_{2}$. For dilute suspensions, the second virial coefficient [158] provides information about the strength of the radially symmetric attraction between spherical particles:

$$
B_{2}=\frac{1}{2} \int \mathrm{d}^{3} r\left(1-e^{-U(r)}\right)=2 \pi \int_{0}^{\infty} \mathrm{d} r r^{2}\left(1-e^{-U(r)}\right)=2 \pi \int_{0}^{\infty} \mathrm{d} r r^{2}(1-g(r)) .
$$

Beyond the ideal gas contribution it determines the leading non-trivial term in the expansion of the pressure $p(\rho) /\left(k_{B} T \rho\right)=1+B_{2} \rho+\ldots$ in terms of powers of the number density $\rho$. In Ref. [67] $B_{2}$ has been calculated for the potential given by Eq. (18) at the state points for which, according to the experiments reported in Ref. [11], aggregation sets in. It turns out that at these states of aggregation onset the values of $B_{2}$ are close to each other and that to a certain extent those $B_{2}$-isolines, which emerge by belonging to these experimental data points, agree with each other and with the possible shape of the aggregation onset line (see Fig. 3). However, based on this analysis one cannot state definitely that $B_{2}$ can serve as a quantitative indicator for the onset of aggregation. Further efforts, both theoretically and experimentally, are needed to provide more precise values of the relevant quantities.

\section{Phase behavior}

In suspensions with sufficiently large packing fractions of colloids, the attraction among them due to CCFs can induce a so-called "liquid-gas" phase separation of the colloids, i.e., the separation of two phases which differ with respect to their colloidal number density. Adopting the effective one-component approach allows one to use standard liquid state 
theory in order to determine the onset of phase separation. Within this effective approach feedback mechanisms of the colloids, acting on the solvent and changing its critical behavior, are neglected. Therefore this approximation does not allow one to describe reliably all details of the full many-component system. However, one can identify certain regions of the thermodynamic phase space for which this approach is applicable. One expects the effective one-component model to work well for temperatures corresponding to the one-phase region of the pure solvent and for an intermediate range of values of the colloid number density $\rho$. The latter should be large enough so that the competition between the configurational entropy and the potential energy due to the effective forces can induce a phase separation, but small enough so that the approximation of using an effective pair potential between the colloids is valid and the influence of the colloids on the phase behavior of the solvent is secondary.

In Ref. [66], for particles interacting via the effective pair potential given in Eq. (18), the phase coexistence curve $T_{c x}^{(\mathrm{eff})}\left(\eta=(4 / 3) \pi R^{3} \rho \mid c_{a}\right)$ with the critical point temperature $T_{c}^{(e f f)}$ and the spinodal (i.e., the loci where, within mean-field theory, the isothermal compressibility $\chi_{T}$ diverges) have been calculated using density functional theory within the so called random phase approximation [80] and the integral equation approach [158]. The results obtained from these two approaches, which are presented in Fig. 4 (Fig. 2 in Ref. [66]), differ only slightly. The loci of the phase separation depends sensitively on the strength $A$ of the repulsive part of the effective pair potential (Eq. (3) ) and the solvent compositions. For solvent compositions, which are somewhat poor in the component preferred by the colloids, even short correlation lengths suffice to bring about phase separation. The critical value $\eta_{c}$ of the colloidal packing fraction $\eta=(4 \pi / 3) R^{3} \rho$ (in terms of number density $\rho$ ) is rather small, i.e., $\eta_{c} \approx 0.07$, because the effective hard sphere diameter $\sigma$, which results from the soft repulsion $U_{r e p}$, is larger than $2 R$. Furthermore, the binodals shown in Fig. 4 are rather flat compared with, e.g., the ones for hard spheres interacting via a short-ranged, attractive, and temperature independent potential. In the present system, the deviation of $T$ from the critical temperature $T_{c}^{(s)}$ which corresponds to a range of $\eta$ for the coexisting phases as large as the one shown in Fig. 4, is about 1\%o, whereas for a system of hard spheres with an additional attractive interaction the corresponding temperature deviation is a few percent. The critical temperature $T_{c}^{(e f f)}$ obtained within DFT agrees with the simple prediction $B_{2}^{*}=B_{2, c}^{*}$, acting as an implicit equation for $T_{c}$, as suggested by Vliegenthart 
and Lekkerkerker [160] and Noro and Frenkel [161] (VLNF), where $B_{2}^{*}(T) \equiv B_{2}(T) / B_{2}^{(H S)}$ is the reduced second virial coefficient and $B_{2, c}^{*}$ is the critical value of $B_{2}^{*}$ for Baxter's model of adhesive hard spheres [162]. $B_{2}^{(H S)}=\frac{2 \pi}{3} \sigma^{3}$ is the second virial coefficient of a suitable reference system of hard spheres (HS) with diameter $\sigma$. The effective hard-sphere diameter can be taken as $\sigma=\int_{0}^{r_{0}}\left(1-\exp (-U(r)) \mathrm{d} r\right.$, with $U\left(r=r_{0}\right)=0$. One can adopt also other definitions of $\sigma$ (for a discussion see Ref. [163]). According to VLNF, $B_{2}^{*}$ is a useful indicator of the occurrence of a phase separation into a colloidal-rich ("liquid") and a colloidal-poor ("gas") phase. An extended law of corresponding states proposed by VLNF predicts that the value of the reduced second virial coefficient $B_{2}^{*}$ at the critical point is the same for all systems composed of particles with short-ranged attractive interactions, regardless of the details of these interactions. This (approximate) empirical rule is supported by experimental data [160] and theoretical results [164.

\section{MULTICOMPONENT MIXTURE}

\section{A. General discussion}

For the kind of systems considered here, the determination of phase equilibria is rather subtle because - due to the concomitant adsorption phenomena, which are state dependent - the effective potential between the colloids depends on the thermodynamic state itself. Experiments have revealed [11, 19] that for suspensions very dilute in colloids and with a phase separated solvent, basically all colloidal particles are populating the phase rich in the component, say $a$, preferred by the colloids (this phase has concentration $c_{a}^{(1)}$ ). This implies that in coexisting distinct phases the effective potential acting between the particles is different. This is not captured by the effective potential approach discussed above. Besides the colloid-solvent also the solvent-solvent interactions can influence the effective potential and, accordingly, the phase behavior of the effective colloidal system. This has been demonstrated by recent MC studies in which various kinds of model solvents have been used [79]. On the other hand, the presence of colloidal particles may alter the phase behavior of the solvent. In the case of molecular fluids, it is well established that the phase diagrams of ternary mixtures, as they emerge from those of binary mixtures by adding a third component, are distorted and become more complex relative to the underlying origi- 
nal binary ones [165, 166]. Similar distortions and complex features of phase diagrams are observed experimentally [16-18] upon adding colloidal particles to binary solvents. In particular, one finds a decrease of the lower critical temperature. These observations tell that for reliably determining such phase diagrams the full, many component mixture has to be considered. The importance of considering the colloidal suspension with a binary solvent as a truly ternary mixture has been already pointed out in Ref. [26]. Taking into account the features of the CCP one can try to predict the expected topology of the phase diagrams for ternary solvent-solvent-colloid mixtures [66]. Upon adding colloids, the two-phase region of the demixed phases of the pure solvent extends into the three-dimensional thermodynamic space of the actual colloidal suspension. At fixed pressure this space is spanned by $T$, the concentration $c_{a}$ of the component $a$ of the binary solvent, and, e.g., by the colloidal number density $\rho$. The actual shape of the two-phase region forming a tube-like manifold is expected to depend sensitively on all interactions present in the ternary mixture (see Fig. 5). The foremost difficulty in describing such mixtures theoretically or in dealing with them via computer simulations consists of the fact that the sizes of their constituents differ by, a few, orders of magnitude. This property distinguishes them significantly from mixtures of hard spheres, needles, and polymers [167, 168], for which the constituents are of comparable size, i.e., none of them is larger by a factor of ten or more than the others. In contrast to molecular ternary mixtures as modeled, e.g., in terms of a lattice gas [169], in colloidal suspensions the colloidal particles influence the other two components not only by direct interactions but also via strong entropic effects. These occur because their surfaces act as confinements to fluctuations of the concentration of the solvent and they also generate a sizeable excluded volume for the solvent particles.

\section{B. Monte Carlo studies}

MC simulations for a lattice model of ternary mixtures, suitably mimicking colloidal particles suspended in a near-critical binary solvent, offer the advantage over other available approaches that they account for both fluctuations and nonadditivity of the emerging CCFs. Due to the large size difference between the colloid and solvent particles and due to the critical slowing down of the ternary mixture upon approaching its critical point, studying the corresponding three-dimensional systems by MC simulations is computationally challenging. 
As a computationally cheaper substitute, two-dimensional lattice models have been treated by this method in Refs. [54, 125, 126]. In a first of such a MC simulation study [125], the colloids have been modeled as discretized hard discs of radius $R$ (in units of the lattice spacing) occupying a fraction $\eta$ of sites on the square lattice. The remaining lattice sites have been taken to be occupied by a solvent molecule of either species $a$ or $b$ with no empty sites left. A similar model of solvent-solvent-colloid mixtures has been considered earlier by Rabani et al. [127] in order to study drying-mediated self-assembly of nanoparticles. In Refs. [125, 126], only the nearest neighbor repulsive interactions between the components of a binary solvent have been considered. This drives phase segregation, which in the absence of colloids has a critical point belonging to the $2 d$ Ising universality class. In order to mimic the preference of the colloids for component $b$ of the binary solvent, a nearest-neighbor attractive interaction between the colloid and component $b$ has been taken into account. It has been assumed that there is only a nearest-neighbor repulsion between the colloid and component $a$. In the limit $\Delta \mu_{s}=\mu_{a}-\mu_{b} \rightarrow \infty$ of the chemical potential difference between the components $a$ and $b$, the ternary mixture reduces to a binary mixture of $2 d$ hard-discs and solvent component $a$, which exhibits coexistence between a fluid and a solid phase. In the study by Edison et al. [125], the preclusion of solvent-mediated colloidal aggregation, arising from complete wetting and capillary condensation, has been implemented by focusing on colloids, which are immersed in a supercritical mixed phase of components $a$ and $b$, such that $b$-rich critical adsorption layers (for critical adsorption profiles at spheres and cylinders see Refs. [96, 170] and references therein) form on the colloid surfaces in equilibrium with a supercritical $a$-rich solvent in the bulk. The careful simulation study in Ref. [126], in which the solvent has been treated grand canonically at constant pressure, has revealed the existence of gas-liquid and fluid-solid transitions, occurring in a region of the thermodynamic variables $\left(\eta, t=\left(T-T_{c}^{(s)}\right) / T_{c}^{(s)}, \Delta \mu_{s}=\mu_{a}-\mu_{b}\right)$ of ternary mixtures which extends well away from the critical point of the solvent reservoir (especially concerning the fluid-solid transitions). It has been found that in all phases the local solvent composition is strongly correlated with the local colloid density. The coexisting colloidal liquid and solid phases are poor in component $a$, whereas in the gas phases coexisting with the liquid or the solid phases the solvent composition is very close to the composition of the solvent reservoir, which, however, is far from its critical composition. These features agree with experimental observations [11, 19] and cannot be captured by an effective one-component approach as 
discussed in the previous section. As expected [171, all pair correlation functions decay exponentially on the scale of the same correlation length. Strikingly, the correlation length found in the homogeneous supercritical state of the ternary mixture was much larger than the colloid radius $(R=6)$, which in turn clearly exceeded the correlation length of the solvent reservoir. Upon adding a small volume fraction of colloids, the gas-liquid critical point of a ternary mixture shifts continuously from that of the colloid-free solvent to the negative values of $\Delta \mu_{s}$, which decrease upon increasing $t$. In addition, the MC simulation data indicate the existence of a second, lower metastable gas-liquid critical point located at larger values of $\eta$. The gas-liquid-solid triple point is also expected to occur near the fluid-solid coexistence of pure hard discs shown as the vertical dashed lines in Fig. 6. The authors of Ref. [126] have checked to which extent a description in terms of an effective pair potential can account for the phase behavior observed in their MC simulations. They have concluded that those approaches, which exclusively employ effective pair potentials, as obtained from, e.g., planar slit studies combined with the Derjaguin approximation, overestimate the extent of gas-liquid coexistence and underestimate how much the critical point of the ternary mixture is shifted relative to that of the solvent reservoir.

In Ref. [54], the two-dimensional Ising model, with embedded colloids represented as disclike clusters of spins with fixed orientation, has been treated by the geometric cluster algorithm (see Sec. II B 3). However, the focus of this study has not been the phase behavior of a ternary mixture but rather the two- and three-body CCP. Employing the geometric cluster algorithm facilitated the study of the same lattice model as in Refs. [125, 126] but in $d=3$ [172]. As anticipated in Refs. [125, 126], the phase diagram displays the same qualitative features as in the two-dimensional case.

\section{Mean field theory}

The mean field approximation of the model considered in Ref. [126] was studied in more detail in Ref. [173]. It is based on free-volume arguments, according to which the Helmholtz free energy is taken as the sum of three contributions, describing the direct interactions among the pure colloids, the pure binary mixture of solvent $a$ and solvent $b$, and the colloid - solvent $b$ mixture. For the pure-colloid contribution the hard-disc free energy has been employed, with its distinct expressions for the fluid [174] and the solid [175] phase. In the 
free space between the colloids, the mean field free energy for the $a-b$ mixture has been taken; the solvent is excluded from the volume of the colloids. Concerning the colloid solvent $b$ contribution, the mean adsorption energy of component $b$ at the colloid surface has been taken. As can be inferred from Fig. 6, the topology of the resulting phase diagram and its temperature dependence agree qualitatively with the one obtained from simulations (see Fig. S2 in the Supplemental Material for Ref. [126] and Fig. 1 in Ref. [173]). In particular, a lower critical point has been found. However, as already expected on the basis of the different spatial dimensions, there is no quantitative agreement; the parameter values used in order to obtain phase diagrams, which are similar to those from simulations, were chosen empirically. It seems that in these studies the underlying mean field approximation severely underestimates the effect of the hard-core repulsion of the colloids. In addition to the colloidal gas and colloidal liquid, this theory predicts the occurrence of two crystal phases which have the same (hexagonal) structure but with different lattice spacings. In the $3 \mathrm{~d}$ thermodynamic state space of a ternary mixture spanned by $\Delta \mu_{s}, \eta$, and $t$ one finds also upper and (metastable) lower colloidal gas-liquid critical lines and a colloidal solid-solid critical line. (The critical points in Fig. 6(a) lie in the planes cutting the full phase diagram at three constant temperatures.) The fluid-solid transition corresponds to a first-order freezing transition. The critical point of the colloid-free solvent $\left(t=0, \Delta \mu_{s}=0, \eta=0\right)$ is shifted considerably upon adding a small amount of colloids (see the red dots in Fig. 6(a)). The authors of Ref. [173] have pointed out that experiments on colloidal aggregation are typically performed by suspending a fixed number of colloids in a solvent at a fixed (pure) solvent composition, which we denote by $c_{a}^{(s)}$, and then by adjusting the temperature of the system to reversibly induce aggregation. They have identified the so-called aggregation line, i.e., the loci of the points $\left(t, c_{a}^{(s)}\right)$ at fixed $\eta$ and $c_{a}^{(s)}$ at which aggregation is observed first, as the line which demarcates the one-phase region of the ternary mixture, which lies at the outside of the line, and the region at its inside where colloidal phase separation can be found. Such a line (see Fig. 7) ends at an $\eta$-dependent critical point of the ternary mixture, which is shifted from the critical point of the pure solvent towards a higher ( $\eta$-dependent) temperature. In the vicinity of their end points these lines are slightly bent, which indicates reentrant dissolution. Such lines qualitatively resemble the experimental results for strong aggregation reported by Beysens and Estève in Ref. [9] (see the solid line in Fig. 3 therein). This tends to support the view on the aggregation lines as the line of onset of colloidal phase 
separation in the full ternary mixture. The above mean field calculation has been performed in $d=2$ [125] and then extended to $d=3$ [173] but no new features of the phase behavior have been found. Although the uncertainties generated by the large differences of length scales prevent a quantitative comparison with the available experimental data, the studies reported in Refs. [125, 173] are very valuable because they reveal the possible scenarios for the phase behavior, which can occur in such ternary mixture, highlighting the physical mechanisms behind it.

\section{EXPERIMENTS}

\section{A. Phase transitions and aggregation in bulk}

The view, that aggregation, as observed in pioneering experiments by Beysens and Estève [9], is in fact a reversible phase transition in a ternary suspension, has been tested via early experimental studies by Wegdam, Schall et al. [176]. In these studies, charge-stabilized polystyrene spheres of radius $R=105 \mathrm{~nm}$ suspended in a mixture of 3-methylpyridine (3MP), water, and heavy water near its lower critical point have been considered. The mass fractions of the components of this latter mixture have been chosen such that the mass density of the solvent mixture closely matches that of the colloidal particles in the region of the parameter space where phase transitions have occurred. If the system is densitymatched, the growth of the nucleated liquid or solid phases is not perturbed by gravity at a very early stage and can be followed until macroscopically large coexisting phases are formed. In order to characterize the phase behavior of the system the authors have used small angle $X$-ray scattering (SAXS). Transition temperatures were also determined by measuring the sample turbidity. Moreover, the samples have been observed directly with a CCD camera. The only information, which has been provided by the authors about the phase diagram of the 3MP-water-heavy water mixture at the mass fractions actually used, is the coexistence temperature $T_{c x}^{(s)} \approx 65^{\circ} \mathrm{C}$ at which this (chemically in fact binary) solvent mixture demixes. The schematic phase diagram shown as Fig. 1(a) in Ref. [176] suggests that the considered mass fraction $\omega_{3 \mathrm{MP}}$ of 3 -methylpyridine investigated in this study (and therein denoted as $\left.c_{3 \mathrm{MP}}\right)$ has been smaller than the critical one. Instead of $t=\left(1-T / T_{c}^{(s)}\right)$, the authors of Ref. [176] have used the deviation $\Delta T=T-T_{c x}^{(s)}$ as the actual control parameter. Upon 
increasing the temperature from a value in the one-phase region of the pure solvent towards $T_{c x}^{(s)}$, the onset of colloid aggregation, at which the system separates into a colloid-rich and a colloid-poor phase as indicated by regions of high and low turbidity and by the appearance of peaks in the structure factor, has been observed at a sharply defined temperature $T_{a}$. Depending on the particle volume fraction of the colloids in the suspension (which is the ratio between the volume of all colloids in the suspension and the volume of the system), two or three phases of the colloidal suspension have been found: at low volume fractions this is a fluid phase in equilibrium with a fcc crystal, whereas at larger volume fractions there is a gas phase in equilibrium with a liquid phase and this liquid phase is coexisting with a fcc solid. Interestingly, upon increasing temperature quickly the measured structure factor indicated the formation of a glassy state as in a molecular system. On the basis of the experimental results reported in Ref. [176], it is not possible to infer uniquely the physical origin of the attractive potential which gives rise to the observed reversible aggregation. The authors concluded that all mechanisms discussed in Sec. III can play a role. Concerning the role of CCFs in this experiment, one finds that the temperature $T_{a}$ of the onset of aggregation is ca. 8-4 K (depending on the volume fraction of the colloids) below $T_{c x}^{(s)}$, which corresponds to $\left(T_{c x}^{(s)}-T_{a}\right) / T_{c x}^{(s)} \simeq 0.02-0.01$, which seems to lie outside the critical region. However, in Ref. [176] the actual critical temperature $T_{c}^{(s)}$ of the solvent is not given; if $T_{c}^{(s)}$ is lower, it is possible that the corresponding values of the reduced temperature actually lie within the critical region. The bulk correlation length of the solvent at $T=T_{a}$ has been estimated from light scattering to be ca. $8 \mathrm{~nm}$, which is somewhat short. However, the experiments were performed at an off-critical composition of 3MP such that along the corresponding thermodynamic path the phase is relatively poor in the solvent component preferred by the colloids. If $T_{a}<T_{c}^{(s)}$, this composition precludes that colloidal aggregation arises from complete wetting and capillary condensation [1]. On the other hand, it is this thermodynamic region where the CCFs are expected to be strongest although their range, governed by bulk correlation length, is smaller than the one at the critical composition. Thus it is rather plausible that CCFs are playing a crucial role in this experiment.

In a subsequent study by Wegdam, Schall et al. [68, the aggregation of colloidal particles suspended in the same (quasi-) binary mixture of 3MP, water, and heavy water has been observed directly by using confocal microscopy. The fluorescent fluorinated latex colloids of radius $R=200 \mathrm{~nm}$ used in this study exhibit affinity for water. In this experiment 
the refractive indices between the colloids and the solvent have been matched closely. Two different compositions of 3MP have been studied, one below (mass fraction $\omega_{3 M P}^{(1)}=0.24$ ) and one above (mass fraction $\omega_{3 M P}^{(2)}=0.37$ ) the critical composition $\omega_{3 M P, c}=0.31$. (In Ref. [68] the critical mass fraction of $3 \mathrm{MP}$ is denoted as $X_{c}$.) The colloid volume fraction has been kept fixed at the rather small value 0.002 and the $\mathrm{D}_{2} \mathrm{O} / \mathrm{H}_{2} \mathrm{O}$ mass ratio was chosen as 0.25 . For the suspension poor in 3MP no aggregation has been observed upon increasing temperature within the homogeneous one-phase region of the solvent until phase separation of the whole mixture has taken place. This is expected to occur for 3MP mass fractions so far off the critical value that the CCFs are negligibly small. Above $\omega_{3 M P, c}$, within a rather wide temperature range of $T_{c x}^{(s)}-T_{a}=8 \mathrm{~K}$, reversible aggregation has been observed. For the very small volume fraction of colloids used, the formation of clusters rather than a colloid phase transition has been observed and the kinetics of this aggregation process has been studied. The clusters have been a few particle diameters wide. The sizes of the clusters, as inferred from the maximum of the confocal image intensity $I(q)$ in Fourier space, have been found to to increase linearly as function of time. The fractal dimension of these aggregates has indicated that the growth process is diffusion limited. Because the particles have desorbed from the clusters at a certain finite rate, the authors have been able to estimate the energy scale of the attraction between the particles by comparing the observed escape frequency with the corresponding attempt frequency, which is the inverse of the Brownian time, i.e., the time in which a particle diffuses a distance equal to its radius, and by assuming that this whole process is thermally activated; this renders $3 k_{B} T$ as the estimate of for the energy scale of attraction. The authors rightfully argued that the CCFs alone are sufficient to induce aggregation in charged-stabilized colloids. In order to show that this is indeed the case for their experiments, they analyzed their data using a simple expression for the effective pair potential between the colloidal particles. As in Eq. (18), it consists of two competing contributions with the repulsive part decaying exponentially on the scale of the Debye screening length $\kappa^{-1}$ (denoted as $\ell_{D}$ in Ref. [68].) Concerning the CCP contribution, a rather simple expression has been adopted. First, although the solvent in the actual experiment has not been at the critical composition, the occurrence of the corresponding relevant scaling variable $\Lambda$ (Eq. (4) ) or, equivalently, $\Sigma$ (Fig. 1) has been neglected. Second, the Derjaguin approximation (Eq. (15)) has been employed with $\mathcal{A}_{+}$ set to 2. As both contributions decay exponentially, the hypothesis was put forward that 
aggregation should occur when both decay lengths, $\xi_{t}$ and $\kappa^{-1}$, become comparable. This hypothesis has been tested by adding salt in order to vary the range of the repulsive Coulomb interactions and by changing temperature in order to vary the critical Casimir attraction. The Debye screening length $\kappa^{-1}$ follows from the formula $\kappa^{-1}=\sqrt{\epsilon \epsilon_{0} k_{B} T /\left(e^{2} \sum_{i} \rho_{i}\right)}$ (see below Eq. (1)) with $\sum_{i} \rho_{i}=2 N_{A} \ell$, where $\left\{\rho_{i}\right\}$ are the number densities of all ions (regardless of the sign of their charges), $N_{A}$ is the Avogadro number, and $\ell$ is the ionic strength (i.e., the molar density of positively charged ions) of the solvent. The bulk correlation length $\xi_{t}$ has been determined independently using X-ray and light scattering. The data representing the onset of aggregation for various values of $\kappa^{-1}$ fit well to the anticipated relation $\xi_{t}(T)=\kappa^{-1}$.

This data analysis has been objected and redone in Ref. [69] with the conclusion that, in fact, most of the data reported in Ref. [68] for the onset of aggregation have been located within ranges of values of $\xi$ and $\kappa$ for which the proposed pair potential does not apply or, in other words, the model proposed in Ref. [68] does not predict aggregation to occur at $\xi_{t}=\kappa^{-1}$. The comment [69] linked to Ref. [68] pointed out the following weaknesses of the theoretical analysis in Ref. [68]. The majority of the experimental data correspond to rather small bulk correlation lengths, i.e., $\xi_{t} \lesssim 5 \xi_{t,+}^{(0)}$, for which the adopted form of the $\mathrm{CCP}$ is not valid and should be complemented by corrections to scaling. Moreover, the effect of screening of the surface charge upon adding salt to the solution, which changes the amplitude $A$ of the repulsive contribution to the pair potential (Eq. (18)), has been neglected. Therefore, as a function of $\xi_{t}$ and $\kappa^{-1}$ the total pair potential exhibits richer forms than the two forms (a purely repulsive potential above the line $\xi_{t}(T)=\kappa^{-1}$ and in addition an attractive potential well below the line $\xi_{t}(T)=\kappa^{-1}$ ) shown in Ref. [68] (see Fig. 8). The model pair potential actually used in Ref. [68] is valid in the range where the distances $D$ between the colloids (denoted as $\ell$ in Refs. [68] and [69]), which are close to the position $D_{\text {min }}$ of the minimum of the total potential, satisfy $R \gg D_{\min } \gtrsim \xi_{t}, \kappa^{-1}$. Together with the requirement that the potential depth should be at least $-3 k_{B} T$, this leads to a range of applicability of the model which is much more stringent than $\xi_{t}(T)<\kappa^{-1}$ as indicated in Refs. [68]. However, the reanalysis presented in Ref. [69] does not exclude the possibility that the observed aggregation is not solely due to the competing effects of repulsive electrostatic and attractive critical Casimir interactions. 


\section{B. Effective colloid-colloid pair potential}

In Refs. [71, 72] the effective pair potential between colloidal particles for a dilute (volume fraction $2 \%$ ) suspension of poly- $n$-isopropyl acrylamid microgel (PNIPAM) particles with a radius of $R=250 \mathrm{~nm}$ has been inferred from the pair correlation function $g(r)$. For a sufficiently dilute suspension of solute, the potential of mean force $V_{m f}(r) \sim-\ln g(r)$ can be identified with the effective pair potential $V(r) ; g(r)$ has been determined from the $2 d$ images obtained by confocal microscopy. The spatial resolution has been estimated to be ca. $0.03 \mu m$ in the image plane and ca. $0.05 \mu \mathrm{m}$ in the direction perpendicular to it. The solvent was, as before, a (quasi) binary mixture of 3MP - water - heavy water with various compositions, including the critical one and compositions slightly off the critical one (towards the $3 \mathrm{MP}$ poor phase). The measurements have been performed for various temperatures upon approaching the solvent two-phase coexistence curve from the homogeneous mixed phase. The obtained pair potential displayed a very soft repulsion at small separations and developed an increasingly deep minimum as $T$ approaches the coexistence temperature $T_{c x}^{(s)}$ of the solvent. At low temperatures, at which $V(r)$ is purely repulsive, the authors have been able to fit $V(r) /\left(k_{B} T\right)$ to the screened electrostatic, exponentially decaying potential $U_{\text {rep }}$ (see Eq. (2)) with plausible values of the parameters. From this the CCP has been then determined by the best fit to the exponential form given in Eq. (15), but with the amplitude and the decay length treated as fit parameters, assuming that the total $U(r)$ is the sum of $U_{C}$ and $U_{r e p}$ (as in Eq. (17)). This assumption is justified for small salt concentrations [177] which has been the case for the samples studied there. (There is experimental [178] and theoretical [177] evidence that at larger salt concentrations the coupling between the charge density and the order parameter can alter significantly the standard critical adsorption and CCFs.) Moreover, it has been assumed that for the temperatures studied, within a range $\Delta T<-1 \mathrm{~K}$, the 'background' repulsive contribution is de facto temperature independent. This data analysis has rendered the length scale for the experimental decay of the CCP, which, however, differs from the bulk correlation length. This discrepancy as well as the softness of the repulsion at small separations have been attributed to the fluffiness of the colloidal particles. The choice of PNIPAM particles has been motivated by their special properties when dissolved in the solvent described above. They swell, which prevents sedimentation, and in this swollen state their refractive index matches that of the solvent. 
In a subsequent paper [72], the fitting procedure has been improved in the sense that the bulk correlation length $\xi_{t}$ has not been anymore an adjustable parameter. Assuming the standard scaling law for $\xi_{t}$ as a function of temperature, the amplitude $\xi_{t,+}^{(0)}$ has been used as a fit parameter in addition to the amplitude and the decay length of $U_{r e p}$. Using one set of these three fit parameters, Dang and coworkers [72] have fitted all experimental data to the sum of two exponentially decaying functions. The fitted analytic expression for the potential has been further used for performing Monte Carlo (MC) simulations of the colloidal sample. The colloidal gas-liquid coexistence has been investigated by using Gibbs Ensemble MC [179] whereas colloidal liquid-solid coexistence has been determined by using Kofke's GibbsDuhem integration technique [180]. The conclusiveness of these MC simulation results for the actual colloidal phase behavior may be questioned because the fits of the experimental data to the analytic expression for the potential are not completely satisfactory. The strongest deviations have occurred at small separations, where the potential has been finite even for center-to-center separations smaller than the diameter of the particles. Moreover, the pair potentials obtained from fitting have not followed the data around the minimum of the total potential, especially close to $T_{c}$. Also these deviations have been attributed to the softness of the PNIPAM particles and to the fact that the scaling variable $\Lambda$ describing off-critical compositions has been neglected. It has been tested if all these deviations may significantly affect the observed phase behavior by calculating the reduced second virial coefficient $B_{2}^{*}=B_{2} / B_{2}^{(H S)}$. Empirically, gas-liquid phase transitions are expected to occur for $B_{2}^{*} \lesssim-1.5$ [160, 161]. Applying this criterion for experimentally determined and fitted potentials has led to an estimate for an upper bound for the deviation $\Delta T=T_{c x}-T_{c x}^{(s)}\left(c_{a}\right)<$ $-0.3 \mathrm{~K}$ of the temperature $T_{c x}$, at which a colloidal gas-liquid transition takes place, from that of the pure solvent phase separation (i.e., $T_{c x}^{(s)}\left(c_{a}\right)$ ), which is in agreement both with the experiments and the simulations reported in Ref. [72]. The comparison between computed and measured phase diagrams at fixed composition of a pure solvent in the plane spanned by $\Delta T=T_{c x}-T_{c x}^{(s)}\left(c_{a}\right)$ and by the colloidal volume fraction (in Ref. [72] denoted as $\phi$ ) is shown in Fig. 9 (Fig. 2 in Ref. [72]). For solutions with off-critical compositions of a (pure) solvent, a reasonable agreement has been found, given the large uncertainties in the experimental determinations of the volume fraction and of $\Delta T$, as well as given the simplified functional form taken for $U_{C}$. On the other hand, for solutions with the (supposedly) critical composition, the agreement is less good. Deviations occur concerning the shape of the 
colloidal gas-liquid coexistence curve, which in simulations is shifted towards values of $\Delta T$ lower than those for the experimental data. Such a shift suggests that the fitted potentials underestimate the attractions between the colloids. According to the authors, this is due to many-body interactions and too small simulation boxes; both are particularly relevant for systems at the critical composition. Another possibility is that the effective one-component approach is not adequate to describe the considered experimental system. Concerning the colloidal liquid-solid phase coexistence in this system, there are no experimental data.

The experimental data for $g(r)$ at a 3MP mass fraction of 0.28 , which is close to the critical value, have been re-analyzed in Ref. [70]. The main improvement over the earlier analyses has been due to a better treatment of the CCP contribution $U(r)=U_{b c k}+U_{C}$ to the total pair potential, where $U_{b c k}$ is a background term. Within this approach, also the dependence of $U_{C}$ on the solvent mass fraction has been taken into account. The scaling function of $U_{C}$ as a function of the scaling variable $\Sigma=\operatorname{sgn}\left(t h_{b}\right) \xi_{t} / \xi_{h}$ (see Eq. (18) and Fig. 11 has been calculated within the Derjaguin approximation by using a local functional approach. In order to minimize the number of fit parameters, the amplitude $\xi_{t,+}^{(0)}$ has been set to the value extracted from the experimental data presented in Ref. [181]. However, the value of the critical mass fraction $\omega_{3 M P, c}$ of the 3MP-heavy water binary liquid mixture is not well established. The inaccuracy of the value for $\omega_{3 M P, c}$ enters into the reduced order parameter $\widetilde{\phi}=\left(\omega_{3 M P, c}-\omega_{3 M P}\right) / \mathcal{B}$, where $\mathcal{B}$ is the non-universal amplitude of the bulk coexistence curve $\omega_{3 M P, c x}(t<0)=\omega_{3 M P, c} \pm \mathcal{B}|t|^{\beta}$. Via the equation of state, which close to the critical point attains its scaling form, this determines the scaling variable $\Sigma=$ $\operatorname{sgn}\left(t h_{b}\right) \xi_{t} / \xi_{h}=\operatorname{sgn}\left(t h_{b}\right) \mathcal{E}_{ \pm}\left(|t|^{\beta} \widetilde{\phi}\right)$, where \pm refers to the sign of $t$. (In Ref. [70] the scaling functions $\mathcal{E}_{ \pm}$have been obtained by using the equation of state within a linear parametric model.) Because $U_{C}$ depends sensitively on $\Sigma$, it has been natural to use the reduced order parameter $\widetilde{\phi}$ as a fitting parameter for achieving the weakest variation of the background potential $U_{b c k}$ with temperature. A fair agreement with the experimental data has been obtained by allowing the value of $\omega_{3 M P, c}$ to differ significantly from the value cited by the authors of the experiment (see Fig. 5 in Ref. [70]). The fit has rendered the background potential which varies slightly with temperature and has an attractive part. This might be due to the the coupling between critical fluctuations and electrostatic interactions and thus deserves further studies.

This detailed knowledge of the CCFs as function of $T$ and $h_{b}$ has been used by the au- 
thors of Ref. [70] to re-analyze the experimental data for the phase segregation, obtained in Ref. [72] and shown in Fig. 9(a). The colloidal gas-liquid coexistence has been calculated within the effective one-component DFT approach using the pair potential $U(r)=U_{b c k}+U_{C}$ fitted to the experimental data as described above, where $U_{C}$ has the scaling form as in Eq. (18). The random-phase (RPA) approximation has been employed for the free energy together with the Percus-Yevick expression for the hard-sphere reference contribution. For the off-critical composition of the solvent, which renders the best expression (i.e., the least temperature dependent one) for $U_{b c k}$ extracted from the experimental data of Ref. [72] (with, however, $U_{b c k}$ still varying slightly with temperature), the phase diagram has been calculated by taking the mean curve of those $U_{b c k}$ which correspond to the various temperatures considered. The corresponding results together with the experimental and MC simulation data from Ref. [72] are shown in Fig. 10. At high colloidal densities, the RPA is in surprisingly good agreement with the experimental data. On the other hand, at low densities the RPA agrees well with the Monte Carlo simulations, while for these densities both approaches underestimate the experimental values which, in turn, agree well with the RPA spinodal. While this latter 'agreement' might be accidental, it nevertheless raises the question whether the experimental system had actually been fully equilibrated at the time of the measurements. As pointed out in Refs. [71, 72, the phase diagrams shown in Fig. 9 are analogous to those of molecular fluids modeled, e.g., by Lennard-Jones or square-well fluids, but with a lower critical point. The peculiarity of the CCP, i.e., the strong temperature dependence of the shape of the potential and of its range, is mirrored by the small temperature range over which colloidal gas-liquid coexistence extends and in the shift of the critical point and of colloidal liquid-solid coexistence to lower volume fractions.

Experience gained from the studies summarized above tells that a meaningful comparison of experimental data and theoretical predictions for the CCP requires accurate experimental knowledge of the solvent bulk phase diagram and of the solvent bulk correlation length. Such corresponding dedicated measurements have been reported in Ref. [86] for PNIPAM particles suspended in a binary liquid mixture of $3 \mathrm{MP}$ and heavy water with addition of $1 \mathrm{mM} \mathrm{KCl}$ salt. The solvents have been prepared with $3 \mathrm{MP}$ mass fractions $\omega_{3 M P}$ (and therein denoted as $c$ and given in units of weight percentage wt $\left.\% 3 \mathrm{MP}=100 \omega_{3 M P}\right)$ ranging from $23.5 \%$ to $33 \%$ around the critical value $\omega_{3 M P, c}^{(s)} \approx 28.0 \%$, and for each of them the temperature has been varied from the homogeneous, mixed phase towards the solvent two-phase coexis- 
tence. In order to achieve intrinsic consistency of the experimental data and to link them to theoretical predictions, the measurements of the pair potentials of the particles in terms of radial distribution functions $g(r)$ have been complemented with dynamic light scattering measurements of the solvent phase diagram and of the bulk correlation length. Below the lower critical temperature $T_{c}^{(s)}$, the solvent correlation lengths have been inferred from the temperature-dependent correlation functions determining the scattered intensity, which are well-described by single-exponential decays with a characteristic time scale $t_{d}$ related to the effective diffusion coefficient $D=\left(q^{2} \mathrm{t}_{d}\right)^{-1}$, where $q$ is the wave vector of the incident wave (equal to $19 \mu^{-1}$ in these specific measurements). This effective diffusion coefficient depends on the linear extent $\xi$ of the correlated regions via a relation analogous to the Stokes-Einstein relation for Brownian particles. However, close to the critical point one has to take into account that $D=D_{\mathrm{c}}+D_{\mathrm{bg}}$ decomposes into a critical and a background part [181], where [181, 182 $D_{\mathrm{c}}=\frac{\mathcal{R} k_{B} T}{6 \pi \eta \xi} K(q \xi)\left(1+b^{2}(q \xi)^{2}\right)^{z / 2}$ and $D_{\mathrm{bg}}=\frac{k_{B} T}{16 \eta_{\mathrm{bg}} \xi} \frac{1+(q \xi)^{2}}{q_{c} \xi}$. Here, $\mathcal{R} \approx 1.05$ is a universal dynamic amplitude ratio [182, 183], $K(x)=3 /\left(4 x^{2}\right)\left[1+x^{2}+\left(x^{3}-x^{-1}\right) \arctan x\right]$ is the Kawasaki function [184], $b=0.55$ [182] is a correction to scaling amplitude and $\eta_{\mathrm{bg}}$ is the background viscosity. The latter has been obtained as a function of $T$ and $\omega_{3 M P}$ by extrapolating the available viscosity data [185] to the critical region. This detailed analysis has allowed one to determine the correlation length for various compositions and to extract the amplitude $\xi_{t,+}^{(0)}=0.44 \mathrm{~nm}$. The phase separation temperatures have been defined as those which correspond the minimum of the diffusion constant for for various values of $\omega_{3 M P}$. They are fitted to the relation $\omega_{3 M P}-\omega_{3 M P, c}=\mathcal{B}|t|^{\beta}$ with the fixed critical exponent $\beta=0.3265$ leading to the estimate $\mathcal{B}=0.6$ for the $\mathrm{OP}$ amplitude. The diameter $2 R=2.12 \mu \mathrm{m}$ of the particles has been deduced by using confocal microscopy whereas their surface charge density $\Upsilon \simeq-0.17 \mathrm{e} \mathrm{nm}^{-2}$ has been obtained from electrophoresis. The experimental radial distribution function $g(r)$, determined by particle tracking, reveals a very soft repulsion, according to which the measured effective potential fulfills $U(r<2 R)>0$ and is very large but not infinite as one would expect for hard core repulsion. As already mentioned in the present section, in the previous studies [71] this softness has been attributed to the fluffiness of the colloidal particles. A plausible alternative explanation for this softness consists of the inaccuracy associated with determining a three-dimensional $g(r)$ from two-dimensional images and, in addition, a certain polydispersity. In Ref. [86] this inaccuracy of the experimental radial distribution function has been compared with the projected theoretical function 
$g_{\text {proj }}\left(r^{\prime}=\sqrt{x^{\prime 2}+y^{\prime 2}}\right)=\int_{-\infty}^{\infty} \mathrm{d} z \int_{-\infty}^{\infty} \mathrm{d} y \int_{-\infty}^{\infty} \mathrm{d} x f_{\left\{0, \sigma_{z}\right\}}(z) f_{\left\{y^{\prime}, \sigma\right\}}(y) f_{\left\{x^{\prime}, \sigma\right\}}(x) g\left(\sqrt{x^{2}+y^{2}+z^{2}}\right)$, where $f_{\left\{x^{\prime}, \sigma\right\}}(x), f_{\left\{y^{\prime}, \sigma\right\}}(y)$, and $f_{\left\{0, \sigma_{z}\right\}}(z)$ are the probability distributions, which account for the uncertainty in the two horizontal directions and the vertical direction, respectively, with the in-plane spreads $\sigma=\sigma_{x}=\sigma_{y}$ being equal. In Ref. [86] these distributions have been taken to be the normal ones. Building upon the consistent description of the bulk properties of the binary solvent, the comparison between the measured pair potentials and the theoretical model, given by Eq. (18) within the Derjaguin approximation for the CCP, would have not required any fitting parameter if the surface of the colloids had exhibited strong preferential adsorption. However, the PNIMP particles are only weakly hydrophilic. In such a case the CCP depends also on the surface field $h_{s}$ via the scaling variable $\hat{h}_{s}=h_{s}|t|^{-\Delta_{1}}$, where $\Delta_{1}$ is the surface counterpart of the bulk gap exponent [87] (see Secs. II B and II B 4). For the film geometry, within mean field theory the dependence on $\hat{h}_{s}$ at the critical concentration $(\Sigma=0)$ can be reduced to a re-mapping $\tilde{\vartheta}_{\|}^{(d)}\left(\mathcal{Y}, \Sigma ; \hat{h}_{s}\right)=s^{d} \vartheta_{\|}^{(d)}\left(s^{-1} \mathcal{Y}, \Sigma\right)$ with a rescaling parameter $s$, which depends on $\hat{h}_{s}$ [143]. The authors of Ref. [86] have found that within the experimentally accessible range of the CCP scaling function, which usually consists of only its exponential tail, one may mimic such a rescaling by using an effective temperature offset $t_{\text {off }}$ which shifts the reduced temperature according to $t^{\prime}=\left(T_{c}-T+\Delta T_{\text {off }}\right) / T_{c}=t+t_{\text {off }}$. Assuming that such a rescaling is valid also beyond mean-field theory, the authors of Ref. [86] have been able to fit all pair correlation functions, for a variety of temperatures and even of off-critical compositions, by taking the single parameter $t_{\text {off }}$ to vary smoothly with composition. Based on these fits the predictions for the pair potential are also given (see Fig. 11). Remarkably, any distorting influence on $g(r)$, which can be described by probability distribution functions which are symmetric about their argument, leaves the second virial coefficient unchanged. This holds because $B_{2}$ is determined by the integrated pair potential (see Eq. (19)). Taking advantage of this property, the authors of Ref. [86] could compare virial coefficients computed from the raw data of $g(r)$ directly with theoretical predictions, without any need to account for experimental inaccuracies and particle polydispersity. The experimental and the theoretical values of $B_{2}^{*}$ show very good agreement in the entire temperaturecomposition plane (see Fig. 12). The comparison based directly on the raw data provides clear evidence that in the investigated solvent composition range it is indeed the critical Casimir interaction which underlies the colloidal attraction. Hence, this direct comparison suggests that not only at the critical composition, but also at these off-critical compositions, 
the attraction is described in terms of a critical Casimir force rather than by wetting effects. Yet, at even larger off-critical compositions, wetting effects are expected to eventually take over and dominate the attraction as observed clearly in Ref. [33].

Using the same experimental imaging and particle tracking techniques, in Ref. [186] the effective interaction potentials for non-spherical dumbbell particles have been extracted from observed radial and angular distributions. The colloidal patchy dumbbell particles have been suspended in heavy water and $3 \mathrm{MP}$ with a mass fraction $\omega_{3 M P}=0.25$ (therein denoted as $\left.c_{3 M P}\right)$. At this subcritical composition, the hydrophobic spherical ends prefer $3 \mathrm{MP}$, while the neck joining these two spherical ends (called by the authors "shell") prefers water. While the one-to-one mapping between radial distribution function and the effective, angularly averaged pair potential still holds for the anisotropic particles, the simple procedure of inferring the effective potential from the radial distribution function is not valid anymore. In order to find an optimal effective potential the authors of Ref. [186] have assumed that dumbbells are the rigid construction of two isotropic spheres each of which interacts via an isotropic pair potential. They have used the following three distinct distribution functions which facilitate the comparison of simulations, theory, and experiment - this way determining that set of potential parameters which renders the best match: (1) the minimum distance radial distribution, for which only the minimum center-to-center distance $r_{i \alpha, j \gamma}$ between two spheres $\alpha$ and $\gamma$ belonging to dumbbell $i$ and $j$, respectively, contributes, (2) the, "site-site" radial distribution between sphere ( called site) $\alpha=1,2$ on dumbbell $i$ and a sphere (site) $\gamma=1,2$ on a different dumbbell $j$, and (3) the bond-angle distribution, which measures the mutual orientation of the particles forming dumbbells. As a first approximation, the observed site-site radial distribution function has been matched to an underlying potential between the dumbbell particles using the so-called reference interaction site model (RISM) in the context of integral equation theory for molecular fluids [187]. Within the latter theory, a molecule is taken to contain several interaction sites so that the total interaction between two molecules is the sum of the site-site interactions. The RISM equation is a generalization of the Ornstein-Zernike equation [188] for hard-core site-site interactions. The authors of Ref. [186] have used the hypernetted chain approximation as a closure. All three measured distributions have been compared with the ones computed using MC simulations of the dumbbell system in which every pair of spheres, apart from the one on the same dumbbell interacts via an effective potential as given by Eq. (18). Because the corresponding experi- 
mental system has not been density matched, the particles sedimented, which was taken into account by including the gravitational potential and a surface field in order to describe the interaction with the bottom wall. Concerning the CCP, the authors of Ref. [186] adopted an oversimplified expression as the one given by Eq. (15), in line with Refs. [68, 71, 72]. As discussed above, such a form is valid only in the limit $D / \xi \rightarrow \infty$ and at the critical composition. Both conditions are not met in the experiment under consideration. Moreover, the amplitude of the bulk correlation length of the solvent and the surface charge, which determines the strength and the decay length of the electrostatic repulsion, respectively, have not been measured. As a consequence, the numerous adjustable parameters have been used to

fit the theoretically proposed effective potentials to the measured ones. Following Ref. [86], the authors of Ref. [186] have used the projected theoretical distribution functions in order to mimic the experimental uncertainties. Nevertheless, the extent of agreement between the measured and the theoretically proposed effective potentials depends on temperature and is not satisfactory. The results for the dumbbell effective pair potentials, based on so many crude approximations and numerous fit parameters, are not conclusive and cannot be predictive. In particular, the quantitative modeling carried out in Ref. [186] is not able to provide the explanation for the observed two ranges of temperatures featuring distinct aggregation behaviors (see Sec. IV C below).

\section{Applications of CCF for self-assembly and aggregation of colloids}

The possibility of controlled aggregation by exploiting CCFs has been used, near the lower critical point of the suspension, for the self-assembly of cadium telluride quantum dots in water - 3MP liquid mixtures with $\mathrm{NaCl}$ salt [189]. By measuring the intensity of scattered light as a function of time and by following the time evolution of the intensity correlation function, it has been found that $1 \mathrm{~K}$ below the phase separation temperature of the suspension the hydrophilic quantum dots with a size of ca. $2.6 \mathrm{~nm}$ form aggregates with an average radius of ca. $700 \mathrm{~nm}$. As expected, for a composition with the 3MP volume fraction being larger than its critical value, i.e., corresponding to the phase poor in the component preferred by the quantum dots, aggregation takes place on shorter time scales and within a larger temperature interval than for a mixture with a 3MP volume fraction being smaller than the critical one. 
Critical Casimir interactions can be utilized for aggregation of colloids being induced by a substrate such that the colloids follow the chemical pattern designed on the surface of a substrate which characterizes the boundary conditions. This is because CCFs respond sensitively to the chemical properties of the confining surfaces. As already discussed in Sec. II], depending on whether the surfaces of the colloid and of the substrate have the same or opposite preferences for the species of the solvent (symmetric or asymmetric boundary conditions), attractive or repulsive CCFs arise. In Refs. [190, 191], charged polystyrene (PS) spheres with $R=1.2 \mu \mathrm{m}$ have been suspended in a water-2,6-lutidine (WL) mixture at its critical composition, i.e., a lutidine mass fraction of $\omega_{L, c} \cong 0.286$. The suspension has been exposed to a chemically patterned substrate with well defined, spatially varying adsorption preferences. This has been achieved by first coating the glass surface with a monolayer of hexamethyldisilazane (HMDS) which rendered the glass surface hydrophobic with a preferential adsorption of lutidine, corresponding to a $(+)$ boundary condition. Spatial patterning of the boundary conditions has been obtained by using a focused ion beam (FIB) of positively charged gallium ( $\mathrm{Ga}$ ) ions which created well-defined hydrophilic (-) areas with a lateral resolution on the order of several tens of nanometers extending over an area of approximately $400 \times 400 \mu \mathrm{m}^{2}$. Close to the lower critical demixing point $T_{c} \approx 307 \mathrm{~K}$ of WL [9], normal and lateral CCFs lead to a strongly temperature dependent attraction between the hydrophilic (-) PS particles and the hydrophilic squares (forming a $2 d$ square lattice of locally symmetric boundary conditions) and to a repulsion from the hydrophobic regions $(+)$ (locally asymmetric boundary conditions). This gives rise to the formation of highly ordered colloidal self-assemblies, the structure of which is controlled by the underlying chemical pattern (see Fig. 13). At higher particle concentrations, additional CCFs between neighboring particles arise and eventually lead to the formation of three-dimensional, facetted colloidal islands on the substrate. In order to quantify lateral CCFs, substrates with a periodic onedimensional chemical pattern have been created forming hydrophilic $(-)$ and hydrophobic (+) stripes with widths of $24.6 \mu \mathrm{m}$ and $5.2 \mu \mathrm{m}$, respectively. For these one-dimensional surface patterns the particle distribution has been measured by digital video microscopy and from the two-dimensional projection of this distribution the effective one-dimensional CCP has been determined by resorting to the Boltzmann factor. In Ref. [191] the experiment has been repeated for substrates with the chemical pattern created by micro-contact printing, which provided sharper, chemical steplike, onedimensional interfaces between alternating 
regions of antagonistic adsorption preferences than the FIB technique does. The reason for the disadvantage of FIB is that the ion beam charges the substrate surface and thus deflects the incoming beam which gives rise to fuzzy chemical steps. It turns out that the agreement between theory and the experimental data is so sensitive (Fig. 13), that the CCP can be used to probe the geometry of the chemical structures, which at present cannot be achieved by other experimental techniques. Accordingly CCFs can be used as a novel surface sensitive probe.

Reversible aggregation of spherical Janus particles has been studied experimentally for micro-sized silica particles half covered by a layer of gold and suspended in the WL mixture at the critical concentration [112]. The gold caps of the particles have been modified chemically by sulfonic groups, which have bestowed a large charge density on their surfaces, leading to an adsorption property which differs from that of the bare silica surfaces. Due to this anisotropy, the particles assembled to clusters with a very specific structure, followed by a hierarchical growth of the clusters. These structures depend on the "valence" of the Janus particle, i.e., the maximum possible number of bonded nearest neighbors. In two spatial dimensions, which applies to the dispersion of the sedimented particles considered in the experiment, the valence is equal to six. Direct visual observation has revealed that particles, randomly dispersed at low temperatures, have started to form micellar structures upon increasing $T$ towards the lower critical point of the WL mixture. Most of the clusters have been trimers and tetramers, with gold-coated hemispheres inside the micelle and silica hemispheres always facing outwards. No "inverted" micelles have been observed, which indicates that the effective attraction between golden patches of colloids has been much stronger than the attraction between the silica parts of the particles. At higher temperatures small clusters, mostly tetramers, have assembled into chain-like structures, finally forming a percolating network. The reversibility of the aggregation, the value of the onset temperature, and the apparent increase of the strength of attraction upon approaching $T_{c}^{(s)}$, have supported the expectation that this aggregation is due to CCFs. The structures of the clusters observed in the experiments are similar to those obtained within MC simulations of hard discs with a pairwise square-well potential acting between the semi-circular patches of the particles. However, the strength of attraction could not be determined from the experimental data. The analysis of the cluster structures corresponding to the lowest internal energy and of the hierarchical clustering suggested the possibility that the self-assembly of Janus particles is 
governed by the valence structure of the clusters and not by that of a single particle.

Recent progress in synthesizing colloidal building blocks allowed the authors of Ref. [192, 193 to fabricate particles with complex shapes and surface patch properties which act as analogues of molecular valence. Multivalent particles, such as dimers, trimers, and tetramers have been produced by swelling and polymerizing clusters of PMMA spheres with a methylmethacrylate/methacrylic acid shell, resulting in geometrically well-defined patches. The specific solvent affinity of the particle patches has been achieved by grafting a polyhydroxy stearic acid-copolymer (PHSA) onto the surface patch, which renders it hydrophobic. The central part of the patchy particles is made hydrophilic by using the ionic initiator potassium persulfate. The particles have been suspended in the homogeneous phase of a binary solvent of heavy water and 3-methylpyridine (3MP) at temperatures below the lower critical temperature $T_{c}=38.55^{\circ} \mathrm{C}$, which has been determined by light scattering and microscopy from solvent phase separation at the critical composition. The solvents were prepared with $3 \mathrm{MP}$ mass fractions $\omega_{3 M P}=0.25$ and 0.31 (therein denoted as $c_{3 M P}$ ), i.e., slightly below and above the critical composition $\omega_{3 M P, c}=0.28$, respectively. The hydrophobic patches have a strong affinity for the non-aqueous component 3MP of the binary solvent, while the hydrophilic central part has an affinity for water. It has been observed that, in solvents poor in the component preferred by the particle patches, the patches approach each other at temperatures close to $T_{c}^{(s)}$, and that dimer particles assemble into directed, chain-like structures. The bending stiffness of the chains has been measured directly by monitoring thermally activated bending fluctuations. In contrast, in 3MP-rich solvents, the particles approach each other sideways resulting in distinct parallel structures. For trimers, the patch-to-patch binding in 3MP-poor solvents leads to staggered chains, while the side-by-side binding in 3MP-rich solvents leads to bent filaments associated with the dense alternating stacking of trimers. In all cases, the assembly is fully reversible as confirmed by the break-up of aggregates upon lowering the temperature several degrees below $T_{c}^{(s)}$. Interestingly, in the case that the chain structure is formed by dimers, upon further approaching $T_{c}^{(s)}$, the authors of Ref. [192] have observed that the chain spontaneously collapses into a compact state, the dimer particles approach each other sideways, and eventually form a close-packed arrangement. It has been argued that in this close-packed state, a particle has more bonding neighbors, and hence a more negative bond energy. The authors of Ref. [192] regard this as being similar to the collapse transition of a polymer, which occurs if the solvent conditions 
go from good to poor. For polymers, too, the reduction of the conformational entropy of the chain is offset by the stronger interparticle interaction energy. Using $\mathrm{MC}$ simulations with the effective pair potential determined in Ref. [186] (see the preceding Sec. IVB), it has been argued in Ref. [192], that the colloidal chain collapse results from the enlarged interaction range due to the increase of the solvent correlation length upon approaching the solvent critical point. In Ref. [193], the effect of patch width on the topology of colloidal aggregates has been experimentally investigated.

\section{Colloidal mixtures}

The way the unique properties of CCFs can be harnessed to manipulate colloidal suspensions has been demonstrated by experiments on colloidal mixtures [73]. This experimental system has been composed of a binary mixture of micro-sized latex particles with slightly different diameters which were suspended in the WL mixture. For this small size difference of the colloids the inherent depletion interaction cannot induce demixing. However, the CCFs can accomplished this, if the two types of particles carry opposite adsorption preferences. The $a$-type particles were functionalized with silane rendering them hydrophobic, i.e., (+) BC, whereas the $b$-type particles had a strong adsorption preference for water, i.e., (-) BC. For such BCs the CCFs among the $a$-type particles and among the $b$-type particles are attractive, whereas between $a$-particles and $b$-particles they are repulsive. At the same temperature and the same distance, the repulsive CCF is stronger than the attractive CCF. In the system under consideration, the van der Waals forces have been eliminated by index matching so that besides the CCFs the only remaining forces have been the screened electrostatic interactions. In the suspension, this mixture of colloids sedimented at the bottom

of the cell forming a dense monolayer. One species has been labeled with a fluorescent dye and traced by using video microscopy. As expected, upon approaching the (lower) critical point of the binary solvent at its critical composition, large structural changes in the colloidal mixture have been observed, signaling the demixing process. It was found that the morphology of this process depends strongly on the mixing ratio $x_{a, b}=\rho_{a, b} /\left(\rho_{a}+\rho_{b}\right)$ of $a$ and $b$-particles, where $\rho_{a, b}$ are the number density of $a$ and $b$ particles (see Fig. 16, which has been taken from Ref. [73]). For $x_{a}=0.54$ and at low temperatures an initially random distribution of particles transforms into a bicontinuous network, which is coarsening further 
upon increasing the temperature. For $x_{a}=0.28$, no bicontinuous structure has been observed; demixing has proceeded via the growth of small clusters of the minority phase (here rich in $a$-particles).

In the theoretical part of this study [73], the effective approach has been employed in order to construct an approximation for the Helmholtz free energy of the colloid mixture. It has been assumed that the effective pair potential $U_{i, j}, i, j=a, b$, can be split into a harddisc part and into a tail, which for identical particles is attractive and for the distinct ones repulsive. For the hard-disc part of the free energy the scaled particle approximation has been used, whereas the attractive or repulsive tail has been treated within the mean field van der Waals approximation. The predictions of this simple theory for the locations of the colloid demixing transitions and their critical point agree fairly well with the experimental data.

\section{E. Effects of depletants on colloidal phase separations}

Piazza and collaborators [77, 78] have studied experimentally the colloidal phase separation in suspensions with a depletion agent, which occurred near the critical point of the depletant enriched solvent. They have used the so-called Hyflon ${ }^{T M}$ MFA latex particles (a copolymer of tetrafluoroethylene (TFE) and perfluoromethylvinylether (PF-MVE); the producer does not provide the meaning of the acronym MFA) of average size $R \sim 90 \mathrm{~nm}$ suspended in water with the nonionic surfactant $\mathrm{C}_{12} \mathrm{E}_{8}$. Salt $(\mathrm{NaCl})$ has been added in order to screen electrostatic interactions between the colloids, caused by their surfaces carrying a negative charge. In addition, this surfactant has provided also steric stabilization of the suspension due to its spontaneous adsorption on the colloid surfaces. What makes this system interesting is, that at concentrations above the critical micellar concentration, $\mathrm{C}_{12} \mathrm{E}_{8}$ in water forms globular micelles with a radius $\sigma=3.5 \mathrm{~nm}$. These micells act as a depletant for the MFA particles. Moreover, the surfactant-water mixture exhibits a liquidliquid phase transition terminating at a lower critical point $\left(T_{c}^{(s)}, c_{s}^{(c)}\right)$ with a very small value of the critical concentration $c_{s}^{(c)}$ of the surfactant $\left(1.8 \%\right.$ mass fraction $c_{s}=m_{s} / m_{t o t}$, where $m_{s}$ is the mass of the surfactant and $m_{t o t}$ is the total mass of the sample). (We use the superscript $s$ as an acronym for the solvent and the subscript $s$ as the one for the surfactant.) Above this critical point two liquid phases exist, one rich and the other poor 
in micelles. The authors have determined, as a function of temperature, the minimum concentration $c_{s}^{(m)}$ of surfactants required to induce colloidal gas-liquid phase separation. The onset of this phase separation has been assumed to manifest itself via the sudden increase of turbidity followed by fast sedimentation of the colloidal particles. The results of these measurements (see Fig. 15, taken from Ref. 78]) show a drastic decrease of $c_{s}^{(m)}$ upon increasing temperature towards the consolute point $\left(T_{c}^{(s)}, c_{s}^{(c)}\right)$ of the surfactant-water mixture, such that $c_{s}^{(m)}$ approaches $c_{s}^{(c)}$ as $T \rightarrow T_{c}^{(s)}$. Far below the surfactant-water miscibility gap, the colloidal phase separation has been obtained due to the action of depletion forces, provided that a sufficient amount of surfactant has been added. The range of the depletion interaction is set by the ratio $\approx 0.03$ between the micelle and the particle size and is very short, but the strength depends on the concentration of the micelles. In Fig. 17, the full $\operatorname{dots}\left(T_{s}^{(m)}, c_{s}^{(m)}\right)$ show the minimum amount $c_{s}^{(m)}$ of surfactant required to induce colloidal phase separation at the temperature $T_{s}^{(m)}$. These points are expected to correspond to the thermodynamic states of the surfactant-water solvent for which the strength of the effective attractive potential between the colloidal particles is roughly constant. The authors have interpreted the reduction in $c_{s}^{(m)}$ observed at higher temperatures as an increase of depletant "efficiency" suggesting that the emerging critical Casimir interactions near the critical point of the surfactant-water mixture can be considered as an extreme case of depletion forces in which the correlated regions of micelles act as a depletant of "renormalized" size, i.e., bigger than that of a micelle, which acts as a bare depletant. However, this interpretation is overstreched because depletion and Casimir forces originate from very different physical mechanisms. In particular, upon approaching the critical point the correlated regions of the emerging phases do not form bubbles but self-similar, bicontinuous structures.

Inspired by these experiments, Sciortino and co-workers [79] have performed a numerical study of the phase separation of hard spheres dispersed in an implicit solvent (i.e., there are no direct interactions, beyond the hard-core ones, between the solvent molecules and these spheres) in the presence of interacting depletant particles. The particles have been taken to interact via the corresponding effective potential $V_{\text {eff }}$ as determined by MC simulations for SW and 3P models of depletants (see Eq. (8) as well as Secs. II and IV E). The phase separation of the colloids as a function of the depletant concentration for various depletant-colloid size ratios has been determined within grand canonical MC simulations. The numerically determined loci of the onset of colloidal gas-liquid phase separation has 
been displayed within the phase diagram of the depletant which exhibits an upper critical point. This revealed that almost for all parameters studied in the MC simulations, the colloidal phase separation induced by CCFs is preempted by the one driven by standard depletion forces. The important message of this study is that only by weakening the attractive depletion interactions, either by lowering the critical depletant concentration $c_{s}^{(c)}$ or by introducing a repulsive interaction between the colloids, it is possible to exploit CCFs for the fine tuning of the self-assembly of colloids in solvents with interacting depletant agents.

\section{F. Aggregation kinetics and structures of aggregates}

Aggregation of colloids in a near-critical binary solvent has been studied experimentally on the ground and under microgravity conditions [68, 85, 194, 195]. The advantage of the critical Casimir effect of providing the ability to tune the effective interactions between the colloids by varying the temperature has been used in order to study systematically the internal structure of the aggregates as a function of their interparticle attraction. Direct visual observations via confocal microscopy of a dilute suspension of latex particles of radius $R$ in a $\mathrm{D}_{2} \mathrm{O} / \mathrm{H}_{2} \mathrm{O}-3 \mathrm{MP}$ mixture have revealed the formation of fractal clusters of colloidal particles near the critical point of the solvent [68]. The analysis of the time-dependent intensity $I(q, \mathrm{t})$ revealed that $I(q, \mathrm{t})$ exhibits a maximum at $q=q^{*}(\mathrm{t})$, which corresponds to the inverse of the mean cluster size. It has been found that in the course of time $q^{*}$ decreases as $q^{*}(\mathrm{t}) \propto \mathrm{t}^{-1}$ and that this linear growth of the size of the clusters with time $\mathrm{t}$ is self-similar. The latter property has been inferred from the data collapse of $I(q, \mathrm{t})$ plotted as a function of the rescaled wave vector $q^{\prime}=q / q_{0}=\left(\mathrm{t} / \mathrm{t}_{0}\right) q R$ with $\mathrm{t}_{0}=\left.33 \mathrm{~min}\right|^{2}$ The fractal dimensions $d_{f}$ determined from $2 d$ projection images have indicated that this system exhibits diffusionlimited cluster aggregation rather than diffusion-limited particle aggregation, for which $d_{f}$ is larger [196]. The fractal dimension reflects the internal structure of an aggregate and relates its radius $R_{g}$ of gyration to the number of particles $N$ according to $R_{g} \propto N^{1 / d_{f}} ; d_{f} \lesssim 3$ corresponds to a close packed structure. $R_{g}$ measures the mean size of the fractal aggregate. It is defined in terms of the mass density $\rho(r)$ of the particles at a distance $r$ from the center of mass of the aggregate as $R_{g}^{2}=\int_{0}^{\infty} r^{4} \rho(r) d r / \int_{0}^{\infty} r^{2} \rho(r) d r$ or, alternatively, based on the pair correlation function $g(r)$ of colloidal particles $R_{g}^{2}=(1 / 2) \int_{0}^{\infty} r^{4} g(r) d r / \int_{0}^{\infty} r^{2} g(r) d r$

\footnotetext{
${ }^{2}$ In the original paper 68] $q^{\prime}$ is given as $q^{\prime}=\left(\mathrm{t}_{0} / \mathrm{t}\right) q R$, which we consider as a typo.
} 
(see, e.g., Ref. [197]). For fractal structures consisting of spheres with spherically symmetric mass density distribution $\rho(r)$, one has $R_{g}=R_{c}$, where $R_{c}$ is the largest radius beyond which the aggregate has zero mass. The images from confocal microscopy have shown that the width of the branches of the clusters is a few particle sizes and that the particles escape from the aggregate at a finite rate. The latter observation and the assumption that this process is thermally activated, allows one to estimate the energy scale of attraction between the particles as ca. $\approx 3 \times k_{B} T$. A more detailed investigation of the structure of the clusters and its evolution was impeded by sedimentation.

In order to avoid this complication, using near field light scattering (NFS), new measurements for the same type of particles have been performed under microgravity conditions on board of the International Space Station and, simultaneously, on the ground [194, 195]. (For these experiments, the composition of the solvent and the volume fraction of the colloidal particles have been taken to be different from those in Ref. [68]. Moreover, different amounts of salt $(\mathrm{NaCl})$ have been added. Thus the results of both studies cannot be compared directly.) The light scattering intensity $I(q, \mathrm{t})$ has provided information about the time evolution of the structure and of the mean size of the aggregates. Its normalized variance, defined as $\left\langle I^{2}(q, \mathrm{t})\right\rangle /\langle I(q, \mathrm{t})\rangle^{2}-1$, with the angular brackets $\langle\cdots\rangle$ denoting the time average, has revealed the onset and the time scale of the aggregation process. The time dependence of the normalized variance results from variations of the number density of the scatters. Accordingly, the start of aggregation has been marked by the time $\mathrm{t}_{a}$ at which this quantity starts to increase from zero. From the slope in the log-log plot of $I(q, \mathrm{t})$ at large momentum transfer $q$, which has been temporally constant during the growth process (which is characteristic of scattering off fractal structures), a fractal dimension has been inferred. It has been found that, upon varying the temperature towards $T_{c}^{(s)}, d_{f}$ has decreased from a value, which is close to the theoretical one of 2.5 for diffusion-limited aggregation, to about 1.8. This indicates that more open structures of clusters are formed if - as expected - the strength of the attraction increases. This has been interpreted to the effect that for an attractive interaction potential with a well depth of ca. $1 \times k_{B} T$ the restructuring of the aggregates into more compact objects can proceed, whereas for deeper attraction wells restructuring gradually stops and the resulting structure is more open. Such more open structures have been observed in the presence of gravity with $d_{f} \simeq 1.6-1.8$ for all temperatures studied. In both cases, i.e., in the presence and absence of gravity, only a 
slight variation with the salt concentration has been found. Moreover, it has been observed that independent of temperature, and thus the strength of the attraction, the aggregates grow similarly. Specifically, the scattered intensity of the growing aggregates as obtained at different times has been reduced to a scaling function $I(q, \mathrm{t}) \approx\left(q_{\text {red }}\right)^{-d_{f}} F\left(q / q_{\text {red }}(\mathrm{t})\right)$, where the time dependent characteristic momentum transfer $q_{\text {red }}(\mathrm{t})$ has been determined from the best fit in the sense of $I(q, \mathrm{t})\left(q_{r e d}\right)^{d_{f}}$ yielding data collapse to a function $F$ of a single variable $q / q_{\text {red }}(\mathrm{t})$. Based on this apparent scaling behavior, an analogy has been drawn between the observed aggregation process and spinodal decomposition processes. For the latter, a similar scaling holds with $d_{f}$ replaced by the spatial dimension $d$ [198]. As for spinodal decomposition, $I(q)$ exhibits a maximum at $q \simeq q_{\text {red }}$ corresponding to the inverse of the characteristic length in the system. In the course of time the momentum $q_{\text {red }}$ shifts to smaller values indicating the growth of this characteristic length. The decrease of $I(q)$ for small values of $q$ reflects the fact that a region around the cluster is depleted of colloids [198]. The growth rate of the characteristic length scale $q_{r e d}^{-1} \propto R_{g} \propto N^{1 / d_{f}}$ of the aggregates has been found to be a power law with exponent $1 / d_{f}$, regardless of the salt concentration. This is characteristic of diffusion-limited models of aggregation [199]. Under gravity, the growth rate has been observed to be significantly faster and nonmonotonic (see Fig. 18, which is taken from Ref. [194]). At early stages it has followed the purely diffusive behavior. After that, aggregation has been influenced strongly by convection of the solvent and by sedimentation. The aggregation among clusters has been found to set in rather fast, resulting in an exponential growth rate, which is characteristic of reaction limited aggregation with a strong dependence on temperature and thus on the strength of the attraction. At still later times, a sudden drop in $q_{\text {red }}^{-1}$ has been observed, which corresponds to the sedimentation of the largest clusters.

Whereas in Ref. [194] the static properties of the aggregates, i.e., their structure factor $S(q)$, have been measured as aggregation proceeds, in the subsequent NFS study of this system [195] the dynamical counterpart of $S(q)$, the intermediate scattering function $\mathfrak{S}(q, \mathrm{t})$ has been measured under microgravity conditions. The aim has been to determine the ratio $\beta \equiv R_{h} / R_{g}$ of the hydrodynamic radius $R_{h}$ of the aggregate and its gyration radius $R_{g}$. This ratio provides information about the density distribution within an aggregate as function of its fractal dimension, which in turn depends on the strength of the particle attraction. The hydrodynamic radius $R_{h}$ of the aggregate is defined through the translational diffusion 
coefficient $D=k_{B} T /\left(6 \pi \eta R_{h}\right)$, where $\eta$ is viscosity of the solvent. For a densely packed spherical aggregate of radius $R_{c}$ and with $d_{f}=3$ one expects $R_{h}$ to be very close to $R_{c}$. The intermediate scattering function $\mathfrak{S}(q, \mathrm{t})$ is the spatial (three-dimensional) Fourier transform of the van Hove distribution function $G(r, \mathrm{t})$, which is the dynamical counterpart of the radial distribution function $g(r)$ [158]. The NSF technique enables one to measure $\mathfrak{S}(q, \mathrm{t})$ instantaneously, on the time scale of the much slower aggregation and diffusion processes. This provides the concurrent measurement of $R_{h}$ from the dynamic and $R_{g}$ from the static structure factor, simultaneously for all accessible wave vectors. $R_{g}$ follows from the FisherBurford expression [200] $S\left(q ; R_{g}\right)=\left(1+\left(2 / 3 d_{f}\right) q^{2} R_{g}^{2}\right)^{-d_{f} / 2}$, which is valid for monodisperse fractal aggregates. $R_{h}$ has been determined via the effective diffusion constant $D_{\text {eff }}$ obtained from the measured decay time $\mathrm{t}_{d}$ of $\mathfrak{S}(q, \mathrm{t}) \propto e^{-\mathrm{t} / \mathrm{t}_{d}}$ via $\mathrm{t}_{d}=1 /\left(D_{\text {eff }} q^{2}\right)$ followed by relating $D_{\text {eff }}$ to $D$ by using $S(q)$ [201]. It has been concluded that in order to obtained reliable data for $R_{h}$, one has to consider the late stages of aggregation, in which the clusters reach sizes large enough to exhibit a well-defined fractal dimension and internal structure and in which the actual polydispersity of the aggregates does not play a significant role. The analysis of the data has shown, that upon approaching the critical point of demixing (i.e., for stronger attraction) the ratio $\beta$ has varied between 0.76 for $d_{f}=1.8$ and 0.98 for $d_{f}=2.5$ at the onset of aggregation (i.e., for weaker attraction). The authors of Ref. [195] have assumed that the fractals are spherically symmetric. Accordingly they have defined the radial density distribution of the fractal objects as $\rho(r)=r^{d_{f}-3} f_{c u t}(r)$, where $r$ is the distance from the center of mass of the cluster and $f_{\text {cut }}$ is a cutoff function which accounts for the finite size of the aggregates. (The exponent follows from the fact that $\rho(r)=\mathcal{N}(r) / V(r) \sim r^{d_{f}-3}$, where $\mathcal{N}(r) \sim r^{d_{f}}$ is the number of particles within a sphere of radius $r$ from the center of the cluster and $V(r) \propto r^{-3}$ is the volume of such sphere.) The expected behavior of the ratio for various forms of a cutoff function has been then compared with the experimental data. It has been observed (see Fig. 19, which is taken from Ref. [195]) that the data for the ratio $\beta$ have been closest to those values which correspond to the assumption of fully compact objects with a Heaviside step function for $f_{\text {cut }}$, independent of the strength of the attraction and of the fractal dimension.

The way to avoid sedimentation for experiments performed on the ground [85] has been to use poly-n-isopropyl acrylanid particles (PNIPAM), which swell in solution. This swelling adjusts their buoyancy, preventing particle sedimentation. This also has allowed the authors 
to observe directly individual particles (after labeling them with a fluorescent dye) even deep in the bulk of the suspension. Using confocal microscopy, the compactness of aggregates of PNIPAM particles, suspended at small volume fractions in the $3 \mathrm{MP}-\mathrm{D}_{2} \mathrm{O} / \mathrm{H}_{2} \mathrm{O}$ mixture, has been studied by determining the number of particles $\mathcal{N}(r)$ within a sphere of radius $r$ from the center of the cluster for quenches with various temperature deviations $\Delta T$ from and below the lower critical point $T_{c}^{(s)}$ of the demixing transition of the solvent. These data have confirmed the fractal character of the structures, i.e., the relation $\mathcal{N}(r) \sim r^{d_{f}}$, with $d_{f}$ decreasing continuously upon increasing temperature towards $T_{c}^{(s)}$ from $d_{f} \approx 3$ to $d_{f} \approx 2.1$. This indicates the formation of more compact structures for weaker attraction (see Fig. 19(h) which is taken from Ref. [85]), in agreement with the behavior observed for the latex particles under microgravity (see the above paragraph). Monte Carlo simulations of diffusion-limited particle aggregation in $d=2$ and $d=3$ have been used in order to relate $d_{f}$ to the attractive effective interaction potential of the particles. Experimentally, the pair potential has been determined from the radial distribution function of the colloids in a way similar to that described in Ref. [71]. The depth $V_{0}<0$ of the attractive well of this pair potential has been related to the quantity $\alpha \propto \exp \left(V_{0} /\left(k_{B} T\right)\right)$ used in the simulations for the probability with which the particles can detach from the growing cluster. The experimental variations of $d-d_{f}$ as a function of $V_{0}$ and of $\alpha$ in simulations exhibit a common curve after suitable rescaling. The authors have interpreted this result as a manifestation of a certain type of universality which occurs although the corresponding simulations have been carried out for different spatial dimensions $(d=2$ and $d=3)$ and although the mechanisms of aggregation differ, i.e., diffusion-limited cluster aggregation in the actual experiments, in which clusters aggregate to form a fractal, and diffusion-limited particle aggregation in simulations, in which a fractal grows by aggregation of single particles.

\section{PERSPECTIVES}

It remains as a challenge to accurately determine the critical Casimir pair potential and its scaling function within the range of the relevant parameters and boundary conditions. This applies to chemically homogeneous or Janus particles of spherical or anisotropic shapes such as cylinders, ellipsoids, cubes, or even more complex shapes such as dummbells or $L$-like ones. Quantitative reliability demands to go beyond mean field theory and the Derjaguin 
approximation. This requires the development of new theoretical approaches and simulation algorithms. Janus particles have already been used in experiments on aggregation in near-critical binary solvents [112]. Colloids with chemically homogeneous or inhomogeneous surfaces, forming patchy colloids [48, 202], with various shapes as well as their mixtures are attractive building blocks for generating a large variety of self-assembled structures by using CCFs. In order to make further progress in understanding the interplay between CCFs and other forces, such as electrostatic ones, for aggregation phenomena, more elaborate and combined experimental and theoretical investigations are required. Concerning the thermodynamic properties of colloidal suspensions with near-critical solvents, a multi-component theory beyond the effective approach and beyond mean field theory is needed. One of the challenges along this line is to construct a suitable theory, which accounts for all relevant degrees of freedom of the solvent and of the solute particles and masters the size differences

of the various species. An interesting line of research would be to study aggregation of colloids in lipid membranes - close to the corresponding lipid phase segregation - or in a liquid analogue of such quasi two-dimensional systems.

[1] R. Evans, J. Phys.: Condens. Matter 2, 8989, 1990.

[2] M. E. Fisher and P. G. de Gennes, C. R. Acad. Sci. Paris Ser. B 287, 207 (1978).

[3] H. G. B. Casimir, Proc. R. Acad. Sci. Amsterdam 51, 793, 1948, online available at the KNAW digital library, http://www.dwc.knaw.nl?DL/publications/PU00018547.pdf.

[4] M. Kardar and R. Golestanian, Rev. Mod. Phys. 71, 1233 (1999).

[5] E. J. W. Varwy and J. T. G. Overbeek, Theory of the Stability of Lyophobic Colloids (Elsevier, Amsterdam, 1948).

[6] C. N. Likos, Phys. Rep. 348, 267 (2001).

[7] S. Asakura and F. Oosawa, J. Chem. Phys. 22, 1255 (1954).

[8] A. Vrij, Pure Appl. Chem. 48, 471 (1976).

[9] D. Beysens and D. Estève, Phys. Rev. Lett. 54, 2123 (1985).

[10] V. Gurfein, D. Beysens, and F. Perrot, Phys. Rev. A 40, 2543 (1989).

[11] (a) P. D. Gallagher and J. V. Maher, Phys. Rev. A 46, 2012 (1992); (b) P. D. Gallagher, M. L. Kurnaz, and J. V. Maher, Phys. Rev. A 46, 7750 (1992). 
[12] M. L. Broide, Y. Garrabos, and D. Beysens, Phys. Rev. E 47, 3768 (1993).

[13] T. Narayanan, J.-M. Petit, M. E. Broide, and D. Beysens, Phys. Rev. E 51, 4580 (1995).

[14] (a) M. L. Kurnaz and J. V. Maher, Phys. Rev. E. 51, 5916 (1995); (b) M. L. Kurnaz and J. V. Maher, Phys. Rev. E 55, 572 (1997).

[15] T. Narayanan, A. Kumar, E. S. R. Gopal, D. Beysens, P. Guenoun, and G. Zalczer, Phys. Rev. E 48, 1989 (1993).

[16] Y. Jayalakshmi and E. W. Kaler, Phys. Rev. Lett. 78, 1379 (1997).

[17] S. R. Kline and E. W. Kaler, Langmuir 10, 412 (1994).

[18] R. D. Koehler and E. W. Kaler, Langmuir 13, 2463 (1997).

[19] (a) H. Grüll and D. Woermann, Ber. Bunsenges. Phys. Chem. 101, 814 (1997); (b) B. Rathke, H. Grüll, and D. Woermann, J. Colloid Interface Sci. 192, 334 (1997).

[20] D. Beysens, J.-M. Petit, T. Narayanan, A. Kumar, and M. L. Broide, Ber. Bunsenges. Phys. Chem. 98, 382 (1994).

[21] D. Beysens and T. Narayanan, J. Stat. Phys. 95, 997 (1999).

[22] B. M. Law, Prog. Surf. Sci. 66, 159 (2001).

[23] C. Bauer, T. Bieker, and S. Dietrich, Phys. Rev. E 62, 5324 (2000).

[24] P. G. de Gennes, C. R. Acad. Sci. (Paris) 292, 701 (1981).

[25] T. Bieker and S. Dietrich, Physica A 252, 85 (1998); 259, 466 (1998).

[26] T. J. Sluckin, Phys. Rev. A 41, 960 (1990).

[27] H. Löwen, Phys. Rev. Lett. 74, 1028 (1995).

[28] R. R. Netz, Phys. Rev. Lett. 76, 3646 (1996).

[29] A. J. Archer, R. Evans, R. Roth, and M. Oettel, J. Chem. Phys. 122, 084513 (2005).

[30] R. Okamoto and A. Onuki, Phys. Rev. E 84, 051401 (2011).

[31] R. Okamoto and A. Onuki, Phys. Rev. E 88, 022309 (2013).

[32] M. Labbé-Laurent, A. Law, and S. Dietrich, J. Chem. Phys. 147, 104701 (2017).

[33] C. Hertlein, L. Helden, A. Gambassi, S. Dietrich, and C. Bechinger, Nature 451, 172 (2008).

[34] A. Gambassi, A. Maciołek, C. Hertlein, U. Nellen, L. Helden, C. Bechinger, and S. Dietrich, Phys. Rev. E 80, 061143 (2009).

[35] U. Nellen, L. Helden, C. Bechinger, EPL 88, 26001 (2009).

[36] S. L. Veatch and S. L. Keller, Biochim. Biophys. Acta, Mol. Cell Res. 1746, 172 (2005).

[37] D. Lingwood and K. Simons, Science 327, 46 (2010). 
[38] A. R. Honerkamp-Smith, P. Cicuta, M. D. Collins, S. L. Veatch, M. den Nijs, M. Schick, and S. L. Keller, Biophys. J. 95, 236 (2008); M. C. Heinrich, I. Levental, H. Gelman, P.A. Janmey, and T. Baumgart, J. Phys. Chem. B 112, 8063, 2008.

[39] E. Gray, J. Karslake, B. B. Machta, and S. L. Veatch, Biophys. J. 105, 2751 (2013); B. B. Machta, E. Gray, M. Nouri, N. L. C. McCarthy, E. M. Gray, A. L. Miller, N. J. Brooks, and S. L. Veatch, Biophys. J. 111, 537 (2016); O. Kimchi and B. B. Machta, Biophys. J. 108, 437 (2015).

[40] B. B. Machta, S. Papanikolaou, J. P. Sethna, and S. L. Veatch, Biophys. J. 100, 1668 (2011).

[41] B. B. Machta, S. Veatch, and J. P. Sethna, Phys. Rev. Lett. 109, 138101 (2012).

[42] J. Benet, F. Paillusson, and H. Kusumaatmaja, Phys. Chem. Chem. Phys. 14, 24188 (2017).

[43] N. Gnan, E. Zaccarelli, and F. Sciortino, Nature Commun. 5, 3267 (2014).

[44] T. Kirkpatrick, J. O. de Zárate, and J. Sengers, Phys. Rev. Lett. 115, 035901 (2015).

[45] C. Cattuto, R. Britto, U. M. B. Marconi, F. Nori, and R. Soto, Phys. Rev. Lett. 96, 178001 (2006).

[46] D. Ray, C. Reichhardt, and C. O. Olson Reichhardt, Phys. Rev. E 90, 013019 (2014).

[47] R. Ni, M. A. C. Stuart, and P. G. Bolhuis, Phys. Rev. Lett. 114, 018302 (2015).

[48] V. D. Nguyen, M. T. Dang, T. A. Nguyen, and P. Schall, J. Phys.: Condens. Matter 28, 04301 (2016).

[49] A. Vrij, E. A. Nieuwenhuis, H. M. Fijnout, and W. G. M. Agtero, Faraday Disc. 65, 101 (1978).

[50] J.-P. Hansen, J. Phys.: Condens. Matter 5, B117 (1993).

[51] M. Dijkstra, R. van Roij, and R. Evans, Phys. Rev. Lett. 81, 2268 (1998); Phys. Rev E 59, $5744(1999)$.

[52] T. G. Mattos, L. Harnau, and S. Dietrich, J. Chem. Phys. 138, 074704 (2013).

[53] T. G. Mattos, L. Harnau, and S. Dietrich, Phys. Rev. E 91, 042304 (2015).

[54] H. Hobrecht and A. Hucht, Phys. Rev. E 92, 042315 (2015).

[55] S. Paladugu, A. Callegari, Y. Tuna, L. Barth, S. Dietrich, A. Gambassi, and G. Volpe, Nature Comm. 7, 11403 (2016).

[56] W. B. Russel, D. A. Saville, and W. R. Schowalter, Colloidal Dispersions (Cambridge University Press, Cambridge, 1989).

[57] J.-P. Hansen and H. Löwen, Annu. Rev. Phys. Chem. 51, 209 (2000). 
[58] J.-L. Barrat and J.-P. Hansen, Basic concepts for simple and complex liquids (Cambridge University Press, Cambridge, 2003).

[59] V. A. Parsegian, Van der Waals forces (Cambridge University Press, New York, 2006).

[60] J. N. Israelachvili, Intermolecular and Surface Forces (Academic, London, 1998).

[61] Y. Levin, Rep. Prog. Phys. 65, 1577 (2002).

[62] M. N. Barber in Phase Transitions and Critical Phenomena, edited by C. Domb and J. L. Lebowitz (Academic, New York, 1983), Vol. 8, p. 145.

[63] V. Privman, in Finite Size Scaling and Numerical Simulations of Statistical Systems, edited by V. Privman (World Scientific, Singapore, 1990), p. 1.

[64] M. Krech and S. Dietrich, Phys. Rev. A 46, 1886 (1992).

[65] A. Pelissetto and E. Vicari, Phys. Rep. 368, 549 (2002).

[66] T. F. Mohry, A. Maciołek, and S. Dietrich, J. Chem. Phys. 136, 224902 (2012).

[67] T. F. Mohry, A. Maciołek, and S. Dietrich, J. Chem. Phys. 136, 224903 (2012).

[68] D. Bonn, J. Otwinowski, S. Sacanna, H. Guo, G. H. Wegdam, and P. Schall, Phys. Rev. Lett. 103, 156101 (2009).

[69] A. Gambassi and S. Dietrich, Phys. Rev. Lett. 105, 059601 (2010).

[70] T. F. Mohry, S. Kondrat, A. Maciołek, and S. Dietrich, Soft Matter 10, 5510 (2014).

[71] V. D. Nguyen, S. Faber, Z. Hu, G. H. Wegdam, and P. Schall, Nature Commun. 4, 1584 (2013).

[72] M. T. Dang, A. V. Verde, V. D. Nguyen, P. G. Bolhuis, and P. Schall, J. Chem. Phys. 139, 094903 (2013).

[73] O. Zvyagolskaya, A. J. Archer, and C. Bechinger, EPL 96, 28005 (2011).

[74] J. Chen and A. Anandarajah, J. Colloid Interface Sci. 180, 519 (1996).

[75] Götzelmann, R. Evans, and S. Dietrich, Phys. Rev. E 57, 6785 (1998).

[76] R. Roth, R. Evans, and S. Dietrich, Phys. Rev. E 62, 5360 (2000).

[77] S. Buzzaccaro, J. Colombo, A. Parola, and R. Piazza, Phys. Rev. Lett. 105, 198301 (2010).

[78] R. Piazza, S. Buzzaccaro, A. Parola, and J. Colombo, J. Phys.: Condens. Matter 23, 194114 (2011).

[79] N. Gnan, E. Zaccarelli, P. Tartaglia, and F. Sciortino, Soft Matter 8, 1991 (2012).

[80] R. Evans, Adv. Phys. 28, 143 (1979).

[81] N. Kern and D. Frenkel, J. Chem. Phys. 118, 9882 (2003). 
[82] C. Hertlein, Messung kritischer Casimir-Kräfte mit TIRM, doctoral thesis, University of Stuttgart (2008); available at http://elib.uni-stuttgart.de/opus/volltexte/2008/3712/.

[83] J. Y. Walz, Curr. Opin. Colloid Interface Sci. 2, 600 (1997).

[84] D. C. Prieve, Adv. Colloid Interf. Sci. 82, 93 (1999).

[85] P. B. Shelke, V. D. Nguyen, A. V. Limaye, and P. Schall, Adv. Mater. 25, 1499 (2013).

[86] S. G. Stuij, M. Labbé-Laurent, T. E. Kogder, A. Maciołek, and P. Schall, Soft Matter 13, 5233 (2017).

[87] H. W. Diehl, in Phase Transitions and Critical Phenomena, edited by C. Domb and J. L. Lebowitz (Academic, New York, 1986), Vol. 10, p. 76.

[88] M. Krech, Casimir Effect in Critical Systems (World Scientific, Singapore, 1994); J. Phys.: Condens. Matter 11, R391 (1999).

[89] J. G. Brankov, D. M. Dantchev, and N. S. Tonchev, The Theory of Critical Phenomena in Finite-Size Systems - Scaling and Quantum Effects (World Scientific, Singapore, 2000).

[90] A. Gambassi, J. Phys.: Conf. Ser. 161, 012037 (2009).

[91] T. W. Burkhardt and E. Eisenriegler, Phys. Rev. Lett. 74, 3189 (1995).

[92] E. Eisenriegler and U. Ritschel, Phys. Rev. B 51, 13717 (1995).

[93] D. Dantchev, F. Schlesener, and S. Dietrich, Phys. Rev. E 76, 011121 (2007).

[94] G. Valchev and D. Dantchev, Phys. Rev. E 96, 022107 (2017).

[95] A. Hanke, F. Schlesener, E. Eisenriegler, and S. Dietrich, Phys. Rev. Lett. 81, 1885 (1995).

[96] S. Yabunaka and A. Onuki, arXiv:1708.08848v1.

[97] F. Schlesener, A. Hanke, and S. Dietrich, J. Stat. Phys. 110, 981 (2003).

[98] E. Eisenriegler and M. Stapper, Phys. Rev. B 50, 10009 (1994).

[99] M. Hasenbusch, Phys. Rev. E 87, 022130 (2013).

[100] J. B. Gilroy, T. Gädt, G. R. Whittell, L. Chabanne, J. M. Mitchels, R. M. Richardson, M. A. Winnik, and I. Manners, Nature Chem. 2, 566 (2010).

[101] A. Walther, M. Drechsler, S. Rosenfeldt, L. Harnau, M. Ballauff, V. Abetz, and A. H. E. Müller, J. Am. Chem. Soc. 131, 4720 (2009).

[102] K. Namba and G. Stubbs, Science 231, 1401 (1986).

[103] Y. Zhao and J. Fang, Langmuir 22, 1891 (2006); J. Pérez-Juste, I. Pastoriza-Santos, L. M. Liz-Marzán, and P. Mulvaney, Coord. Chem. Rev. 249, 1870 (2005).

[104] S. Kondrat, L. Harnau, and S. Dietrich, J. Chem. Phys. 131, 204902 (2009). 
[105] M. Tröndle, S. Kondrat, A. Gambassi, L. Harnau, and S. Dietrich, EPL 88, 40004 (2009); M. Tröndle, S. Kondrat, A. Gambassi, L. Harnau, and S. Dietrich, J. Chem. Phys. 133, 074702 $(2010)$.

[106] M. Labbe-Laurent, M. Tröndle, L. Harnau, and S. Dietrich, Soft Matter 10, 2270 (2014).

[107] M. Labbe-Laurent and S. Dietrich, Soft Matter 12, 6621 (2016).

[108] A. Walther, M. Drechsler, S. Rosenfeldt, L. Harnau, M. Ballauff, V. Abetz, and A. H. E. Müller, J. Am. Chem. Soc. 131, 4720 (2009); N. Prasad, J. Perumal, C.-H. Choi, C.-S. Lee, and D.-P. Kim, Adv. Funct. Mater. 19, 1656 (2009); G.-R. Yi, D. J. Pine, and S. Sacanna, J. Phys.: Condens. Matter 25, 193101 (2013).

[109] F. Sciortino, A. Giacometti, and G. Pastore, Phys. Rev. Lett. 6, 2510 (2009); L. Hong, A. Cacciuto, E. Luijten, and S. Granick, Nano Lett. 6, 2510 (2006).

[110] W. Li, Y. Liu, G. Brett, and J. D. Gunton, Soft Matter 8, 6027 (2012); Y. Liu, W. Li, T. Perez, J. D. Gunton, and G. Brett, Langmuir 28, 3 (2012).

[111] L. Viry, C. Mercader, P. Miaudet, C. Zakri, A. Derre, A. Kuhn, M. Maugey, and P. Poulin, J. Mater. Chem. 20, 3487 (2010); K. Jiang, J. Wang, Q. Li, L. Liu, C. Liu, and S. Fan, Adv. Mater. 23, 1154 (2011).

[112] Y. Iwashita and Y. Kimura, Soft Matter 9, 10694 (2013).

[113] Y. Iwashita and Y. Kimura, Soft Matter 10, 7170 (2014).

[114] E. Eisenriegler, J. Chem. Phys. 121, 3299 (2004).

[115] G. Bimonte, T. Emig, and M. Kardar, EPL 104, 2101 (2013).

[116] M. E. Fisher and P. J. Upton, Phys. Rev. Lett. 65, 2402 (1990); ibid 3405 (1990).

[117] M. E. Fisher and H. Au-Yang, Physica A 101, 255 (1980).

[118] Z. Borjan and P. J. Upton, Phys. Rev. Lett. 81, 4911 (1998).

[119] Z. Borjan and P. J. Upton, Phys. Rev. Lett. 101, 125702 (2008).

[120] R. Okamoto and A. Onuki, J. Chem. Phys. 136, 114704 (2012).

[121] K. K. Mon, Phys. Rev. B 39, 467 (1989); K. K. Mon and K. Binder, Phys. Rev. B 42, 675 (1990)

[122] H. Hobrecht and A. Hucht, EPL 106, 56005 (2014).

[123] A. O. Vasilyev, Phys. Rev. E 90, 012138 (2014).

[124] C. H. Bennet, J. Comput. Phys. 22, 245 (1976). 
[125] J. R. Edison, N. Tasios, S. Belli, R. Evans, R. van Roij, and M. Dijkstra, Phys. Rev. Lett. 114, $038301(2015)$.

[126] N. Tasios, J. R. Edison, R. van Roij, R. Evans, and M. Dijkstra, J. Chem. Phys. 145, 084902 $(2016)$.

[127] E. Rabani, D. R. Reichman, P. L. Geissler, and L. E. Brus, Nature 426, 271 (2003).

[128] J. R. Errington, Phys. Rev. E 67, 012102 (2003).

[129] R. H. Swendsen and J. S. Wang, Phys. Rev. Lett. 58, 86 (1987).

[130] U. Wolff, Phys. Rev. Lett. 62, 361 (1989).

[131] D. P. Landau and K. Binder, A Guide to Monte Carlo Simulations in Statistical Physics 3rd edition (Cambridge University Press 2009).

[132] J. R. Heringa and H. W. J. Blöte, Phys. Rev E 59, 4976 (1989).

[133] B. Derjaguin, Kolloid Zeitschrift 69, 155 (1934).

[134] M. Krech, Phys. Rev. E 56, 1642 (1997).

[135] O. Vasilyev, A. Gambassi, A. Maciołek, and S. Dietrich, EPL 80, 60009 (2007).

[136] O. Vasilyev, A. Gambassi, A. Maciołek, and S. Dietrich, Phys. Rev. E 79, 041142 (2009).

[137] M. Hasenbusch, Phys. Rev. B 85, 174421 (2012).

[138] M. Hasenbusch, Phys. Rev. B 82, 104425 (2010); ibid 85, 174421 (2012).

[139] F. Parisen Toldin and S. Dietrich, J. Stat. Mech.: Theory and Experiment, P11003 (2010);

F. Parisen Toldin, M. Tröndle, and S. Dietrich, Phys. Rev. E 88, 052110 (2013).

[140] M. Hasenbusch, Phys. Rev. E 91, 022110 (2015).

[141] O. Vasilyev, A. Maciołek, and S. Dietrich, Phys. Rev. E 84, 041605 (2011).

[142] M. Hasenbusch, Phys. Rev. B 83, 134425 (2011).

[143] T. F. Mohry, A. Maciołek, and S. Dietrich, Phys. Rev. E 81, 061117 (2010).

[144] O. A. Vasilyev and S. Dietrich, EPL 104, 60002 (2013).

[145] P. Anzini and A. Parola, Phys. Rev. E 94, 052113 (2016).

[146] R. Leidl and H. Wagner, J. Chem. Phys. 98, 4142 (1998).

[147] P. Tarazona, Phys. Rev. A 31, 2672 (1985).

[148] W. A. Curtin and N. W. Ashcroft, Phys. Rev. A 32, 2909 (1985).

[149] A. Parola and L. Reatto, Adv. Phys. 44, 2909 (1995).

[150] M. Fukuto, Y. F. Yano, and P. Pershan, Phys. Rev. Lett. 94, 135702 (2005).

[151] S. Rafaï, D. Bonn, and J. Meunier, Physica A 386, 31 (2007). 
[152] R. Evans, and J. Stecki, Phys. Rev. B 49, 8842 (1994).

[153] D. B. Abraham and A. Maciołek, Phys. Rev. Lett. 105, 055701 (2010).

[154] D. B. Abraham and A. Maciołek, EPL 101, 2006 (2013).

[155] S. Fisk and B. Widom, J. Chem. Phys. 50, 3219 (1969).

[156] N. Gnan, E. Zaccarelli, and F. Sciortino, J, Chem. Phys. 137, 084903 (2012).

[157] N. Fuchs, Z. Phys. Chem. 171, 199 (1934).

[158] J. P. Hansen and I. R. McDonald, Theory of Simple Liquids (Academic, London, 1986).

[159] C. Caccamo, Phys. Rep. 274, 1 (1996).

[160] G. A. Vliegenthart and H. N. W. Lekkerkerker, J. Chem. Phys. 112, 5364 (2000).

[161] M. G. Noro and D. Frenkel, J. Chem. Phys. 113, 2941 (2000).

[162] R. J. Baxter, J. Chem. Phys. 49, 2770 (1968).

[163] H. C. Andersen, J. D. Weeks, and D. Chandler, Phys. Rev. A 4, 1597 (1971).

[164] (a) J. Largo and N. B. Wilding, Phys. Rev. E 73, 036115 (2006); (b) G. Foffi and F. Sciortino, Phys. Rev. E 74, 050401(R) (2006); (c) P. Orea and Y. Duda, J. Chem. Phys. 128, 134508 (2008); (d) D. Gazzillo, J. Chem. Phys. 134, 124504 (2011).

[165] R. J. L. Andon and J. D. Cox, J. Chem. Soc., 4601 (1952); J. D. Cox, J. Chem. Soc., 4606 (1952).

[166] B.V. Prafulla, T. Narayanan, and A. Kumar, Phys. Rev. A 46, 7456 (1992).

[167] M. Schmidt and A. R. Denton, Phys. Rev. E 65, 021508 (2002).

[168] M. Schmidt, J. Phys.: Condens. Matter 23, 415101 (2011).

[169] D. Mukamel and M. Blume, Phys. Rev. A 10, 610 (1974); J. Sivardière and J. Lajzerowicz, Phys. Rev. A 11, 2090 (1975).

[170] A. Hanke and S. Dietrich, Phys Rev. E 59, 5081 (1999); G. Flöter and S. Dietrich, Z. Phys. B 97, 213 (1995).

[171] R. Evans, R. J. F. Leote de Carvalho, J. R. Henderson, and D. C. Hoyle, J. Chem. Phys. 100, 591 (1994).

[172] N. Tasios and M. Dijkstra, J. Chem. Phys. 146, 134903 (2017).

[173] J. R. Edison, S. Belli, R. Evans, R. van Roij, and M. Dijkstra, Mol. Phys. 113, 2546 (2015).

[174] A. Santos, M. López de Haro, and S. Bravo Yuste, J. Chem. Phys. 103, 4622 (1995).

[175] D. A. Young and B. J. Alder, J. Chem. Phys. 70, 473 (1979). 
[176] H. Guo, T. Narayanan, M. Sztuchi, P. Schall, and G. H. Wegdam, Phys. Rev. Lett. 100, 188303 (2008).

[177] F. Pousaneh and A. Ciach, J. Phys.: Condens. Matter 23, 412101 (2011); M. Bier, A. Gambassi, M. Oettel, and S. Dietrich, EPL 95, 60001 (2011); F. Pousaneh, A. Ciach, and A. Maciołek, Soft Matter 8, 3567 (2012).

[178] U. Nellen, J. Dietrich, L. Helden, S. Chodankar, K. Nygard, J. F. van der Veen, and C. Bechinger, Soft Matter 7, 5360 (2011).

[179] D. Frenkel and B. Smit, Understanding Molecular Simulations: From Algorithms to Applications, 2nd ed. (Academic, San Diego, CA, 2001).

[180] D. A. Kofke, J. Chem. Phys. 98, 4149 (1993).

[181] C. M. Sorensen and G. A. Larsen, J. Chem. Phys. 83, 1835 (1985).

[182] H. C. Burstyn, J. V. Sengers, J. K. Bhattacharjee, and R. A. Ferrell, Phys. Rev. A 28, 1567 (1983); H. C. Burstyn, and J. V. Sengers, Phys. Rev. A 25, 448 (1982).

[183] S. K. Das, J. V. Sengers, and M. E. Fisher, J. Chem. Phys. 127, 144506 (2007).

[184] K. Kawasaki, in Phase Transitions and Critical Phenomena, edited by C. Domb and J. L. Lebowitz (Academic, New York, 1986), Vol. 5A, p. 165.

[185] A. Oleinikova, L. Bulavin, and V. Pipich, Int. J. Thermophys. 20, 889 (1999).

[186] A. C. Newton, T. A. Nguyen, S. J. Veen, D. J. Kraft, P. Schall, and P. G. Bolhuis, Soft Matter 13, 4903 (2017).

[187] D. Chandler and H. C. Andersen, J. Chem. Phys. 57, 1930 (1972).

[188] L. S. Ornstein and F. Zernike, Proc. Acad. Sci. Amsterdam 17, 793 (1914).

[189] E. Marino, T. E. Kodger, J. Bart ten Hove, A. H. Veders, and P. Schall, Solar Energy Materials and Solar Cells 158, 154 (2016).

[190] F. Soyka, O. Zvyagolskaya, C. Hertlein, L. Helden, C. Bechinger, Phys. Rev. Lett. 101, 208301 (2008).

[191] M. Tröndle, O. Zvyagolskaya, A. Gambassi, D. Vogt, L. Harnau, C. Bechinger, and S. Dietrich, Mol. Phys. 109, 1169 (2011).

[192] T. A. Nguyen, A. Newton, S. J. Veen, D. J. Kraft, P. G. Bolhuis, and P. Schall, Adv. Mater. 29, 1700819 (2017).

[193] T. A. Nguyen, A. Newton, D. J. Kraft, P. G. Bolhuis, and P. Schall, Materials (Basel) 10, $1265(2017)$. 
[194] S. J. Veen, O. Antoniuk, B. Weber, M. A. C. Potenza, S. Mazzoni, P. Schall, and G. H. Wegdam, Phys. Rev. Lett. 109, 248302 (2012).

[195] M. A. C. Potenza, A. Manca, S. J. Veen, B. Weber, S. Mazzoni, P. Schall, and G. H. Wegdam, EPL 106, 68005 (2014).

[196] J. R. Dutcher and A. G. Maragoni, Soft Materials: Structure and Dynamics (Marcel Dekker, New York, 2004).

[197] W. van Saarloos, Physica 147A, 280 (1987).

[198] M. Carpineti and M. Giglio, Phys. Rev. Lett. 68, 3327 (1992).

[199] T. A. Witten and L. M. Sander, Phys. Rev. B 27, 5686 (1983); P. Meakin, J. Colloid Interface Sci. 102, 491 (1984).

[200] M. E. Fisher and R. J. Burford, Phys. Rev. 156, 583 (1967).

[201] M. Y. Lin, H. M. Lindsay, D. A. Weitz, R. Klein, R. C. Ball, and P. Meakin, Phys. Rev. A 41, 2005 (1990)

[202] N. A. Garcia, N. Gnan, and E. Zaccarelli, Soft Matter 13, 6051 (2017). 


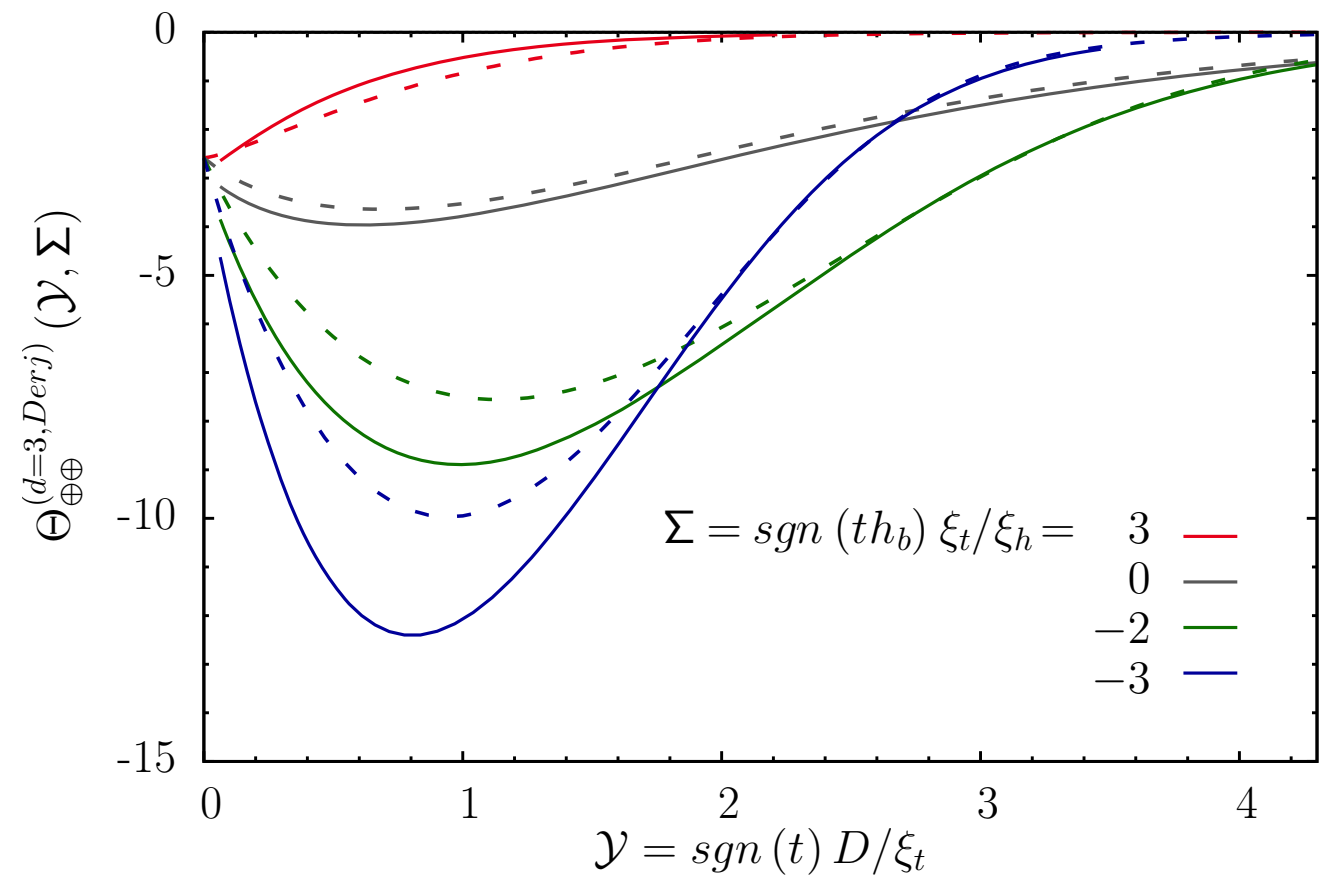

FIG. 1. Scaling function $\Theta_{\oplus \oplus}^{(d=3, D e r j)}(\mathcal{Y}, \Sigma) \simeq(D / R) U_{c}\left(D ; t, h_{b}, R\right)$ (see Eq. 4 and the main text) of the sphere - sphere CCP, as obtained within the Derjaguin approximation by using the extended de Gennes-Fisher functional (solid lines) and the "dimensional" approximation (dashed lines) as a function of the surface-to-surface distance $D$ (in units of $\xi_{t}$ ) for several values of the scaling variable $\Sigma=\Lambda / \mathcal{Y}=\operatorname{sgn}\left(t h_{b}\right) \xi_{t} / \xi_{h}$ related to the bulk ordering field $h_{b}$. At fixed temperature in the onephase region of the solvent $(t>0)$, the CCP is shorter-ranged and much weaker for $h_{b}>0$ (i.e., $\Sigma>0$ ), which favors the same $(+)$ phase of the solvent as the one preferred by the colloid surfaces, whereas $h_{b}<0$ (i.e., $\Sigma<0$ ) favors the other phase. 


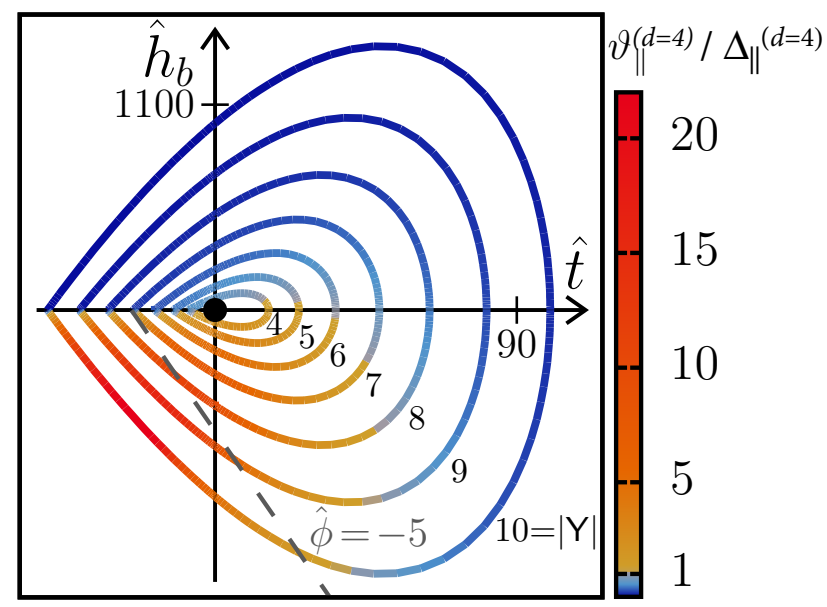

FIG. 2. Normalized mean-field CCF scaling function $\vartheta_{\|}^{(d=4)}(\mathrm{Y}, \Sigma)=D^{4} f_{C}^{\|} /\left(k_{B} T \mathcal{S}\right)$, where $\mathrm{Y}=$ $\operatorname{sgn}(t) D / \xi\left(t, h_{b}\right)$ and $\Sigma=\operatorname{sgn}\left(t_{b}\right) \xi_{t} / \xi_{h}$, for films (of thickness $D$ and macroscopically large crosssectional area $\mathcal{S}$ ) along isolines of constant scaling variable $\mathrm{Y}=4,5, \ldots, 10$ (from the inner to the outermost ring) in the thermodynamic state space of the solvent spanned by $\hat{t}=\left(D / \xi_{t,+}^{(0)}\right)^{1 / \nu} t$ and $\hat{h}_{b}=\left(D / \xi_{h}^{(0)}\right)^{\beta \delta / \nu} h_{b}$ (see Fig. 1 in Ref. [70]); $\xi\left(t, h_{b}\right)$ is the bulk correlation length of the solvent with $\xi_{t}=\xi\left(t, h_{b}=0\right)$ and $\xi_{h}=\xi\left(t=0, h_{b}\right)$. (Note that depending on the particular thermodynamic path under consideration, representations of the scaling function of the critical Casimir force can be more convenient in terms of other scaling variables, such as in Fig. 1 where $\mathcal{Y}=\operatorname{sgn}(t) D / \xi_{t}$ is chosen. The color along the lines of constant $\mathrm{Y}$ indicates the absolute value $\left|\vartheta_{\|}^{(d=4)} / \Delta_{\|}^{(d=4)}\right|$. The bulk critical point of the solvent $\left(\hat{t}, \hat{h}_{b}\right)=(0,0)$ is indicated by $\bullet$. The region shown here lies above the capillary transition critical point, where the film coexistence line ends. For $(+,+)$ boundary conditions as considered here, the capillary condensation transition occurs for $\hat{t}<0$ and $\hat{h}_{b}<0$. The dashed line indicates the path of constant order parameter $\phi$ of the solvent $\hat{\phi}=\frac{D}{\xi_{t,+}^{(0)}} \phi / \mathcal{B}=-5$, where $\mathcal{B}$ is the non-universal amplitude of the bulk OP $\phi=\mathcal{B} t^{\beta}$. Within mean-field theory $\nu=\beta=1 / 2$ and $\nu /(\beta \delta)=1 / 3 . \Delta_{\|}^{(d=4)}=\vartheta_{\|}^{(d=4)}(\mathrm{Y}=0, \Sigma=0)$. 


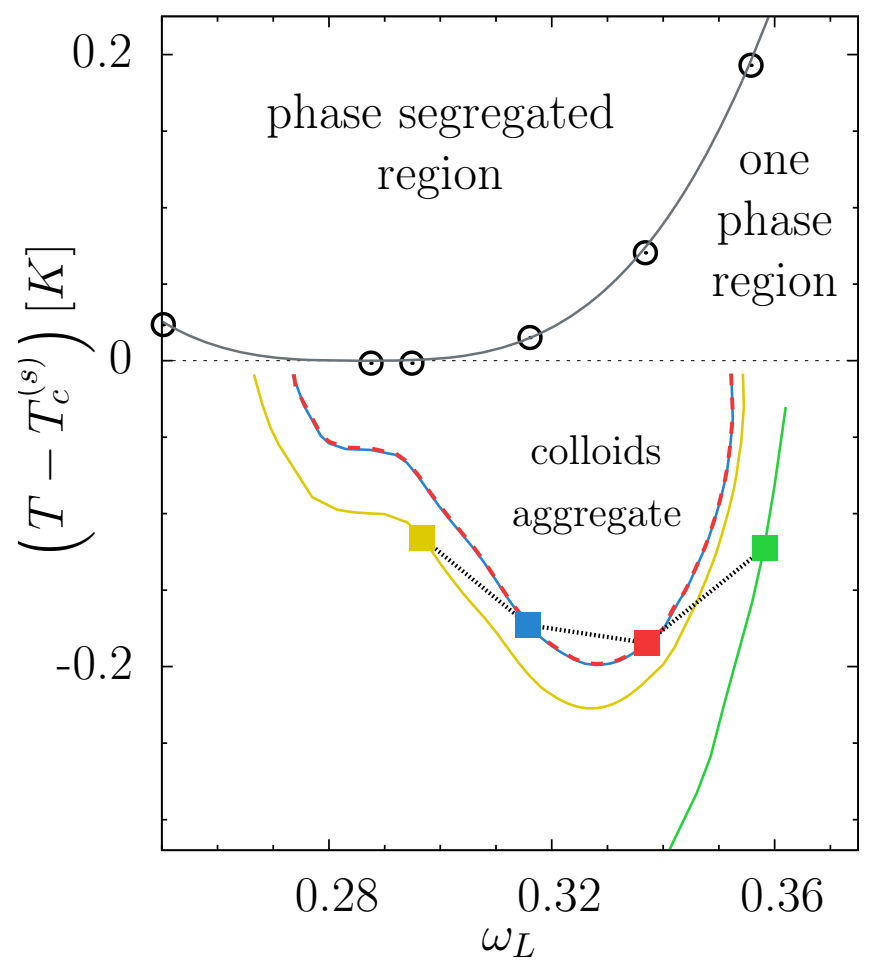

FIG. 3. The experimentally determined coexistence points $[\odot]$ of the binary liquid mixture lutidine-water [11] which exhibits a lower, continuous demixing phase transition. $T_{c}^{(s)}$ is the critical temperature of this demixing transition and $\omega_{L}$ is the mass fraction of lutidine. These coexistence points on the binodal of phase segregation agree well with the relation $\left|\omega_{L}-\omega_{c, L}\right|=B_{\omega}|t|^{\beta}$, where $\beta=0.3265$ and $B_{\omega}=0.765$ (dark gray line). Squares denote the experimentally obtained state points of the onset of aggregation (the straight black dotted lines in between are a guide to the eye) taken from the middle of Fig. 1 in Ref. [11(b)]. Each isoline of constant $B_{2}$ (full, colored lines; for visibility of the blue line the red one is dashed, both lines nearly coincide) corresponding to one of the state points (squares), is calculated by using the effective potential given by Eq. (18); their values are $B_{2} /\left(\frac{4 \pi}{3} R^{3}\right)=-67$ (red square), -65 (blue square), -23 (yellow square), and 5.4 (green square) (Fig. 8 in Ref. [67]). Each $B_{2}$-isoline can capture some qualitative trends of the possible shape of the line of onset of aggregation. The blue and the red lines reveal agreement, but the yellow and the green one not. 

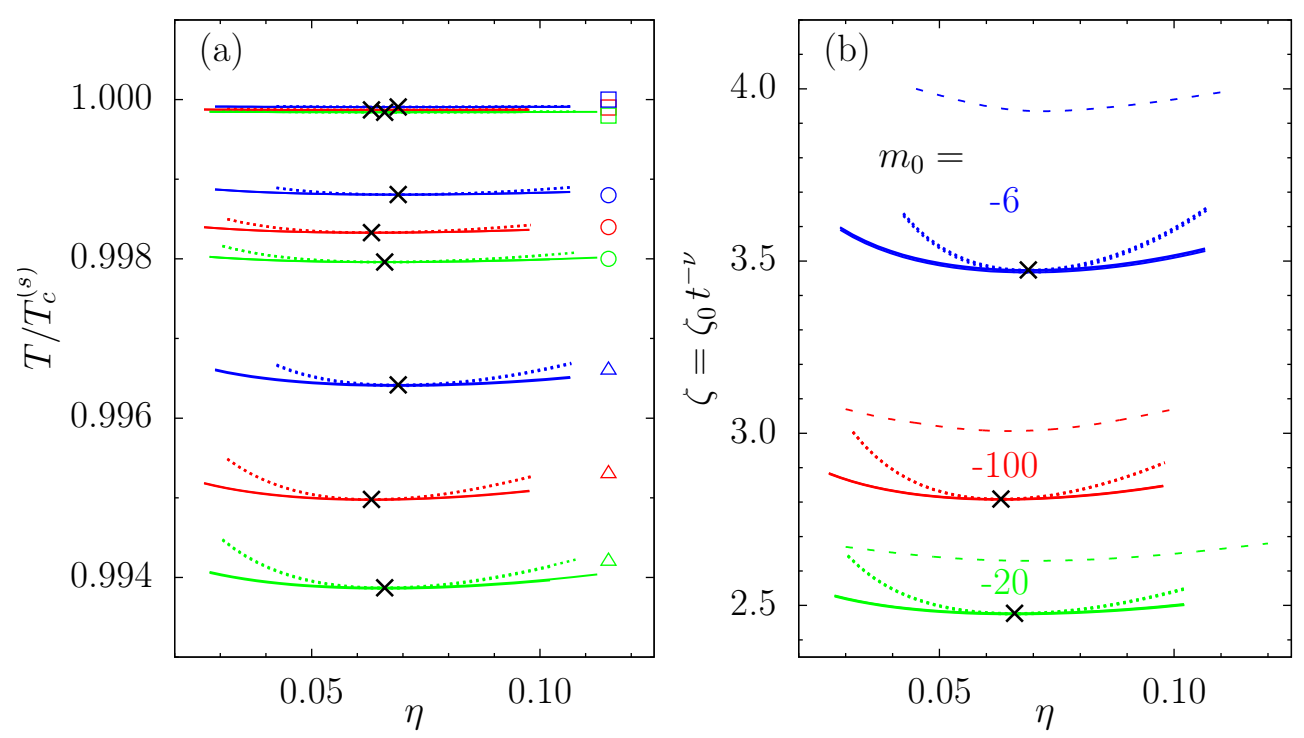

FIG. 4. In the plane spanned by temperature and colloidal packing fraction $\eta$ : (a) colloidal gasliquid phase coexistence curves $T_{c x}^{(\mathrm{eff})}\left(\eta=(4 / 3) \pi R^{3} \rho \mid c_{a}\right)$ (full lines, see, c.f., Fig. 5), spinodals (i.e., loci of mean-field divergence of the isothermal compressibility $\chi_{T}$, dotted lines), and the critical points $T_{c}^{(\text {eff })}$ (crosses) of an effective, one-component system of large colloidal particles as obtained by density functional theory (Fig. 2 in Ref. [66]). These particles of radius $R$ interact via an effective potential given by Eq. 18 with parameters $\kappa R=10$ and $A=1000$ [66] taken at $T_{c}^{(s)}$ ). The curves correspond to a solvent with a lower critical temperature $T_{c}^{(s)}$ and with various fixed solvent compositions $c_{a}$ (Eq. (5)) represented by the variable $m_{0}=\operatorname{sgn}(\phi)\left(\zeta_{0}\right)^{1 / \nu}|\mathcal{B} / \phi|^{1 / \beta}=-100$ (red), -20 (green), -6 (blue). $\mathcal{B}$ is defined via the shape of the solvent binodal $\phi=\mathcal{B} t^{\beta}$ and $\zeta_{0}=\kappa \xi_{t,+}^{(0)}$ (see below). The bulk critical exponents used here are $\nu=1 / 2$ and $\beta=1 / 2$. Close to the phase separation of the solvent the dominant temperature dependence within the effective approach described by Eq. 19) is that of the critical Casimir forces (CCFs), encoded in $\zeta\left(t=1-T / T_{c}^{(s)}\right)=$ $\operatorname{sgn}(t) \kappa \xi_{t}(t)=\operatorname{sgn}(t) \zeta_{0}|t|^{-\nu}$. Therefore, if the temperature (see panel (a)) is expressed in terms of $\zeta$ (see panel (b)) the members of each set of curves with equal color in (a), corresponding to various values of $\zeta_{0}\left(\zeta_{0}=0.01(\square), 0.05(\circ)\right.$, and $\left.0.1(\triangle)\right)$ fall de facto on top of each other and are characterized by $m_{0}$. The dashed lines in (b) correspond to the spinodals determined within the integral equation approach. (Due to $m_{0} \sim\left(c_{a}-c_{a, c}^{(s)}\right)^{-1}$, the quantities $m_{0}= \pm \infty$ correspond to the critical composition $c_{a, c}^{(s)}$. For solvent compositions which are somewhat poor in the component preferred by the colloids, i.e., for intermediate negative values such as $m_{0} \simeq-20$, the critical Casimir forces are strongly attractive. Therefore, for them short correlation lengths suffice to bring about phase separation; accordingly the binodals occur at small values of $\zeta$. Here only thermodynamic states of the solvent which are in the one-phase region, i.e., for $t>0$, are considered. 


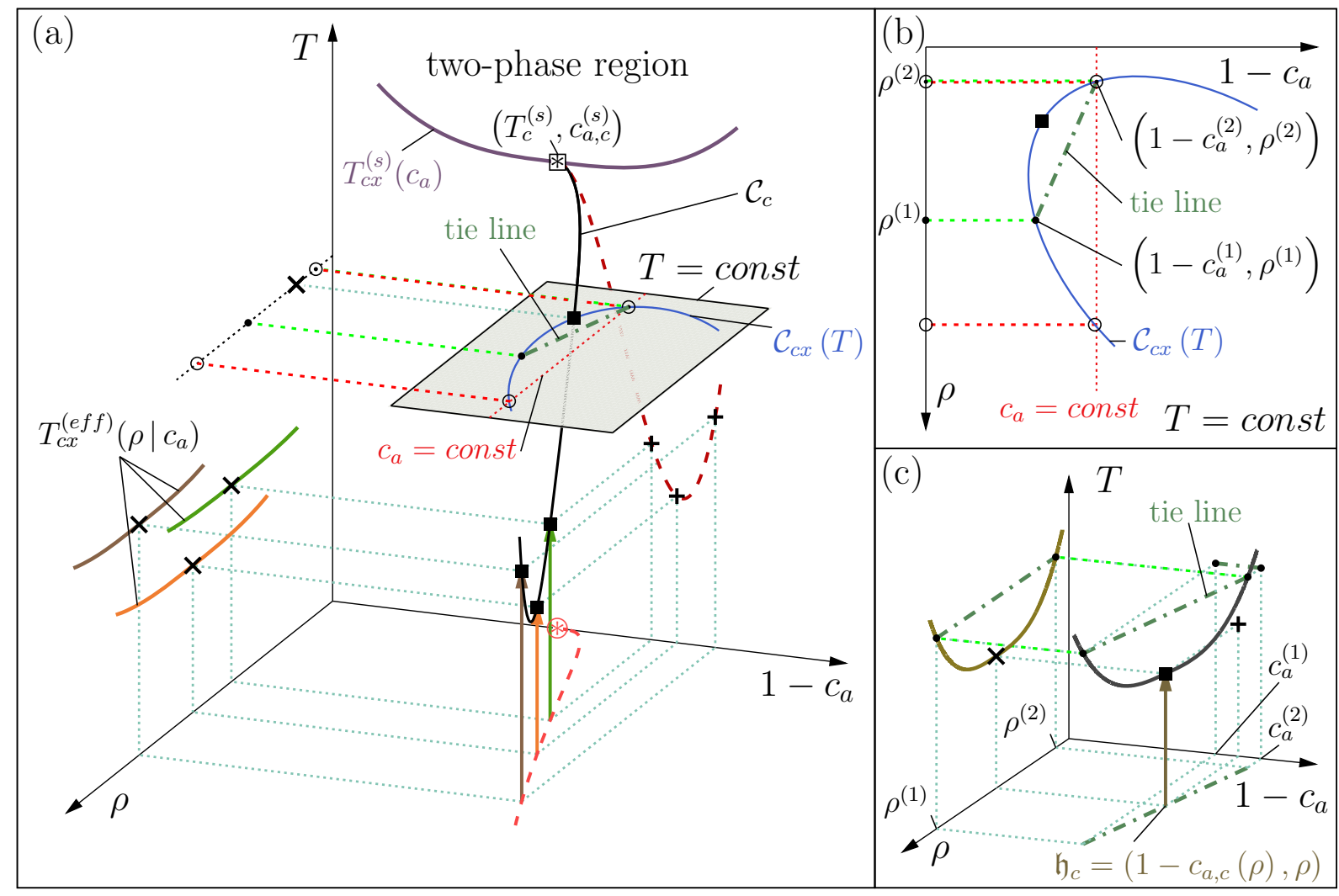

FIG. 5. Sketch of the phase diagram for colloids immersed in a binary liquid mixture at fixed pressure corresponding to a liquid state of the system (Fig. 1 in Ref. [66]). Upon adding colloids the phase separation curve $T_{c x}^{(s)}\left(c_{a}\right)$ of a pure solvent in the $\left(T, c_{a}, \rho=0\right)$ plane extends to a tube-like two-phase region $T_{c x}\left(c_{a}, \rho\right)$ in the $3 d$ thermodynamic (td) space spanned by the temperature $T$, the concentration $c_{a}$, and the colloidal number density $\rho$. The lower critical point $\left.⿴ 囗 T_{c}^{(s)}, c_{a, c}^{(s)}, \rho=0\right)$ of a pure solvent extends to a line $\mathcal{C}_{c}$ (black curve) of critical points (some of which are shown as black squares). Its shape reflects the fact that the $2 d$ manifold $T_{c x}\left(c_{a}, \rho\right)$ of coexisting states is not straight but bent and twisted due to the specific properties of the critical Casimir potential. The red dashed curves denote the projections of $\mathcal{C}_{c}$ onto the planes $\left(\rho, c_{a}\right)$ and $\left(T, c_{a}\right)$. Within the effective one-component approach the coexistence curves are explicit functions of $\rho$ only and depend parametrically on the overall concentration $c_{a}$. All three panels show that in general for $T=$ const the coexisting phases (i.e., the points connected by a tie-line) differ with respect to both $\rho$ and $c_{a}$. Thus the effective one-component approach has a limited applicability for determining the phase diagram. Experimentally or within suitable, sufficiently rich models, upon increasing temperature (along thermodynamic paths indicated by vertical arrows) one is able to determine a coexistence curve (black line in (c)) in the $3 d$ td space. For a description of further details see the caption of Fig. 1 in Ref. [66]. 

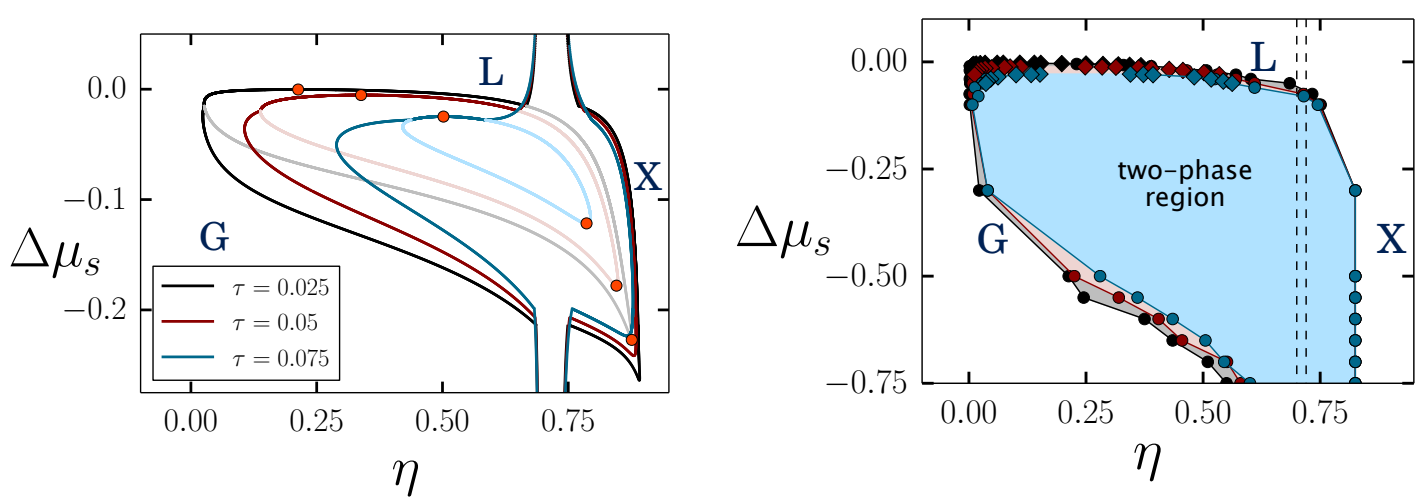

FIG. 6. Phase diagrams of the full ternary colloid - solvent $a$ - solvent $b 2 d$ lattice model (Fig. S2 in Ref. [125]) for three values of $t=\left(1-T / T_{c}^{(s)}\right.$ ) (in Ref. [125] denoted by $\tau$ ): 0.025 (black), 0.05 (dark red), and 0.075 (blue) in the $\left(\Delta \mu_{s}, \eta\right)$ plane of the solvent chemical potential difference and the colloidal packing fraction. The quantity $\Delta \mu_{s}=\mu_{a}-\mu_{b}$ is the chemical potential difference between species $a$ and $b$ (in units of the solvent-solvent interaction strength). The upper panel shows results obtained within mean field theory. The grey, pale red, and pale blue curves correspond to metastable colloidal gas-liquid (G-L) coexistence, which also terminates at the critical point. For each $\tau$ the upper (stable) and lower (metastable) gas-liquid critical points are indicated by red dots. $\mathrm{X}$ denotes the solid phase. (The various phases are inferred from monitoring their free energies.) The lower panel shows the corresponding phase diagrams as determined by Monte Carlo simulations. The diamonds and dots denote the phase boundaries as obtained from grand canonical staged insertion $\mathrm{MC}$ simulations and $\left(\Delta \mu_{s} /\left(k_{B} T\right), \eta, \tau\right)$ ensemble $\mathrm{MC}$ simulations, respectively. Their color corresponds to the value of $\tau$ given in the upper panel. The blue area corresponds to the twophase region for $\tau=0.075$; for $\tau=0.05$ and 0.025 the two-phase regions encompass the previous regions and have added (colored) slices. The vertical dashed lines denote fluid-solid coexistence for pure colloidal hard discs. $T_{c}^{(s)}=T_{c}^{M F T}$ and $T_{c}^{(s)}=T_{c}^{M C}$ ) is the critical temperature of the binary $(a b)$ solvent within mean field theory and MC simulations, respectively. 


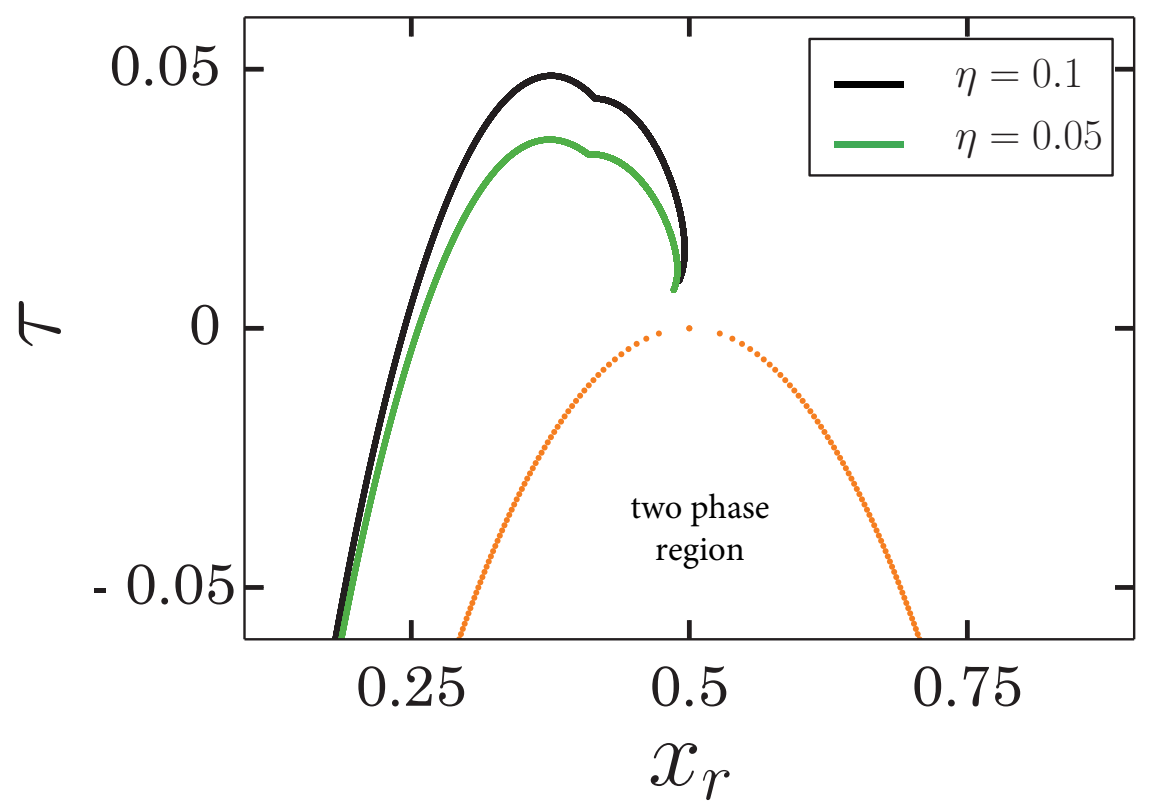

FIG. 7. Aggregation lines (black and green) for a ternary colloid - solvent $a$ - solvent $b$ mixture (interpreted as a colloidal condensation transition), as determined within mean field theory for the lattice model considered in Ref. [125] (Fig. 4 in Ref. [173]), plotted in the reduced temperature composition of the pure solvent in the reservoir $\left(t=\left(1-T / T_{c}^{(s)}\right), c_{a}\right)$ representation (in Ref. [173] denoted by $\left.\left(\tau=\left(T-T_{c}^{M F T}\right) / T_{c}^{M F T}, x_{r}\right)\right)$. A point on the aggregation line at $x_{r}$ gives the temperature at which phase separation of colloids is observed first, upon cooling the suspension at a fixed packing fraction $\eta$ of colloids and for fixed solvent composition $x_{r}$. The $2 d$ calculations have been performed for a parameter $v_{c}$ (approximately equal to the area of the colloidal disc) chosen to be equal to $1000 a^{2}$ (in order to reproduce most closely the MC simulations results of Ref. [125]), where $a$ is the lattice spacing, for the coupling strength between colloid and solvent species $b$ equal to 32 (in units of the solvent - solvent interaction strength), and for two fixed values $\eta=0.1$ and $\eta=0.05$ of the colloid packing fraction. The colloids interact with the members of solvent species $a$ and with each other via a hard core repulsion. The dotted orange line is the binodal of the colloid-free $a b$ solvent. Each aggregation line ends at a critical point of the ternary mixture, which is removed from the binodal of the solvent reservoir (see the main text). The origin of the break in slopes of the aggregation curves is not discussed in Ref.7173. The bent of the aggregation lines near their critical points implies the occurrence of reentrant dissolution upon cooling. 


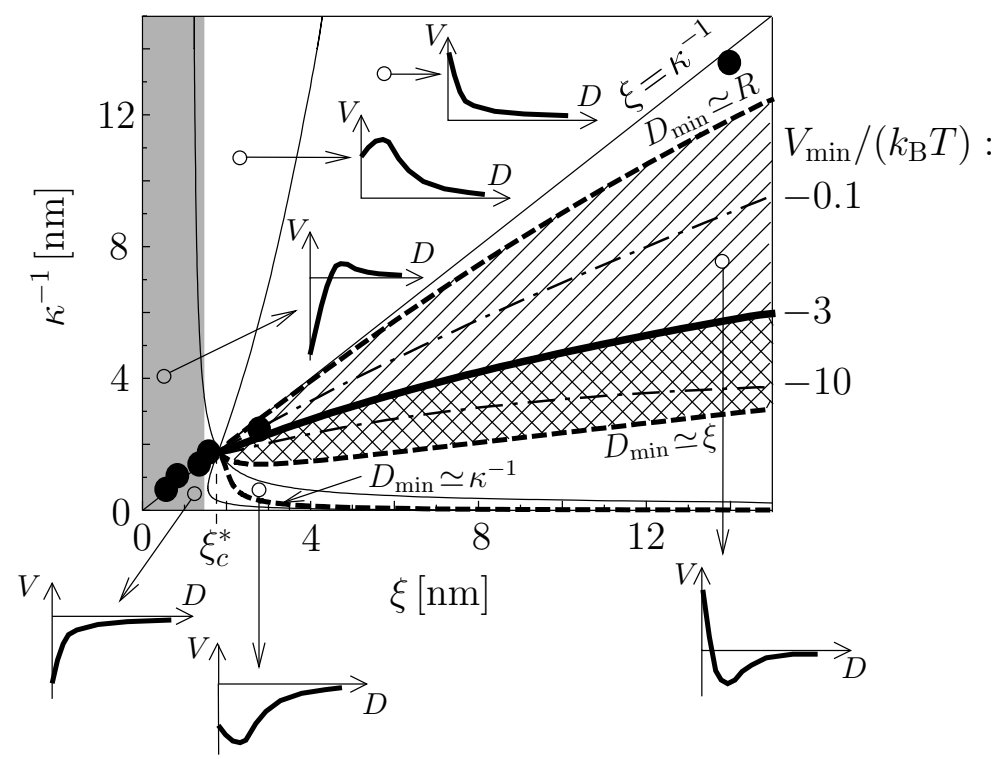

FIG. 8. Shapes of the effective total potential $V(D)=V_{e l}(D)+V_{C}(D)$ of the force acting on two identical colloids of radius $R$ for various values of the bulk correlation length $\xi=\xi\left(t, h_{b}=0\right)=\xi_{t}$ and the Debye screening length $\kappa^{-1}$, where $D$ is the surface-to-surface distance of the two colloids (Fig. 1 in Ref. [69]). Six different regions of distinct shapes of $V(D)$ are limited by the thin solid lines. These lines meet at $\kappa^{-1}=\xi_{t} \equiv \xi_{c}^{*}$. $D_{\min }$ is the position $V_{\min }$ of the minimum of $V(D)$. Within the hatched area enclosed by the thick dashed lines the condition $R \gg D_{\min } \gtrsim \xi_{t}, \kappa^{-1}$ is satisfied. The additional requirement that $V_{\min } \lesssim-3 k_{B} T$ is fulfilled in the cross-hatched part of the hatched are. The black dots mark the experimentally determined [68] aggregation line. For further details see Ref. [69] and Ref. 1 therein. 

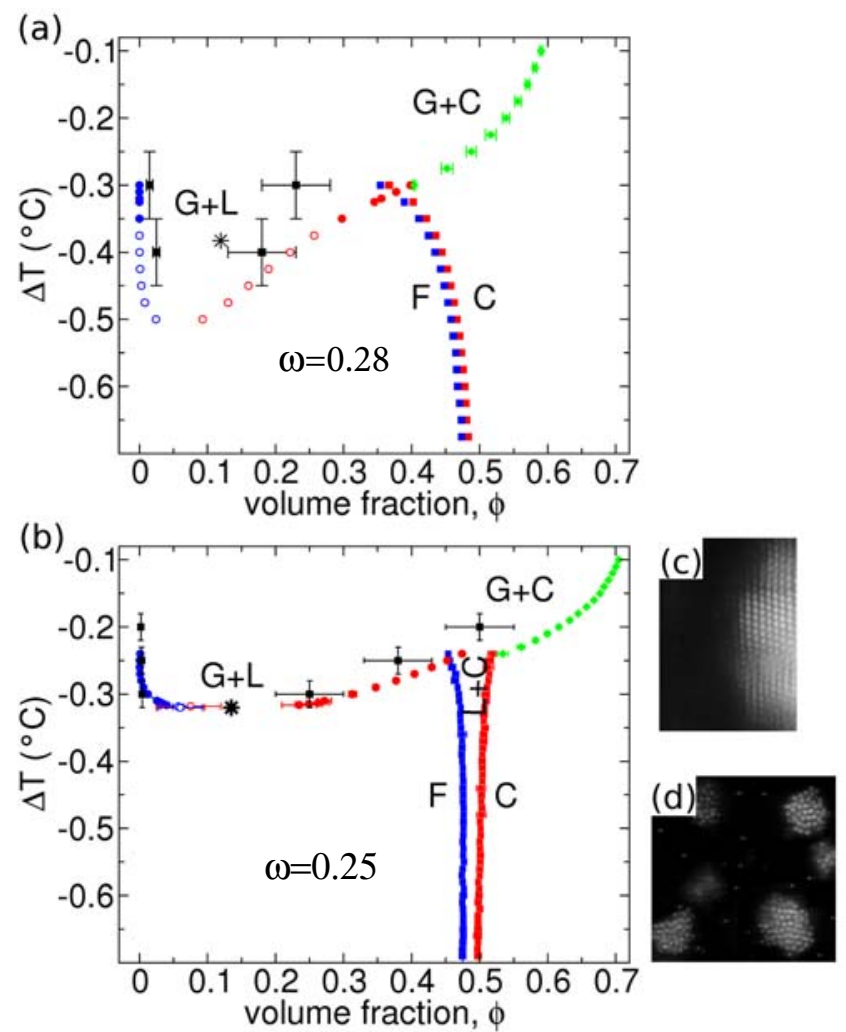

FIG. 9. Phase diagram in terms of $\Delta T=T-T_{c x}^{(s)}$ and volume fraction, in Ref. [71] denoted as $\phi=V_{s} / V_{t o t}$, where $V_{s}$ is the volume of colloids and $V_{t o t}$ is the total volume of the sample. These data have been obtained from MC simulations (colored symbols) for the effective one-component colloidal system governed by a pair potential which is the sum of repulsive and attractive, exponentially decaying, functions describing screened electrostatic and critical Casimir interactions (Fig. 2 in Ref. [71]). G, L, F, and C denote colloidal gas, liquid, fluid, and crystal phases, respectively. G+L, $\mathrm{G}+\mathrm{L}$, and $\mathrm{L}+\mathrm{C}$ stand for the gas-liquid, gas-crystal, and the liquid-crystal coexistence regions. Black squares with error bars are experimental data for PNIPAM particles suspended (a) in the $3 \mathrm{MP}$ - heavy water mixture at the critical $3 \mathrm{MP}$ mass fraction $\omega_{3 M P}=0.28 \simeq \omega_{3 M P, c}$ and $(\mathrm{b})$ in the $3 \mathrm{MP}$ - water - heavy water mixture at the off-critical $3 \mathrm{MP}$ mass fraction $\omega_{3 M P}=0.25$. The authors of Ref. [71] interpret the snapshots of confocal microscopy images for the mixture in (b) as the coexistence of colloidal gas and crystal at $\Delta T=-0.2$ (c) and colloidal gas and liquid at $\Delta T=-0.3(\mathrm{~d})$. Stars indicate the experimental position of the colloidal gas-liquid critical point as estimated by using the law of rectilinear diameters. In (a) the star appears as being placed at a too small value of $|\Delta T|$. 

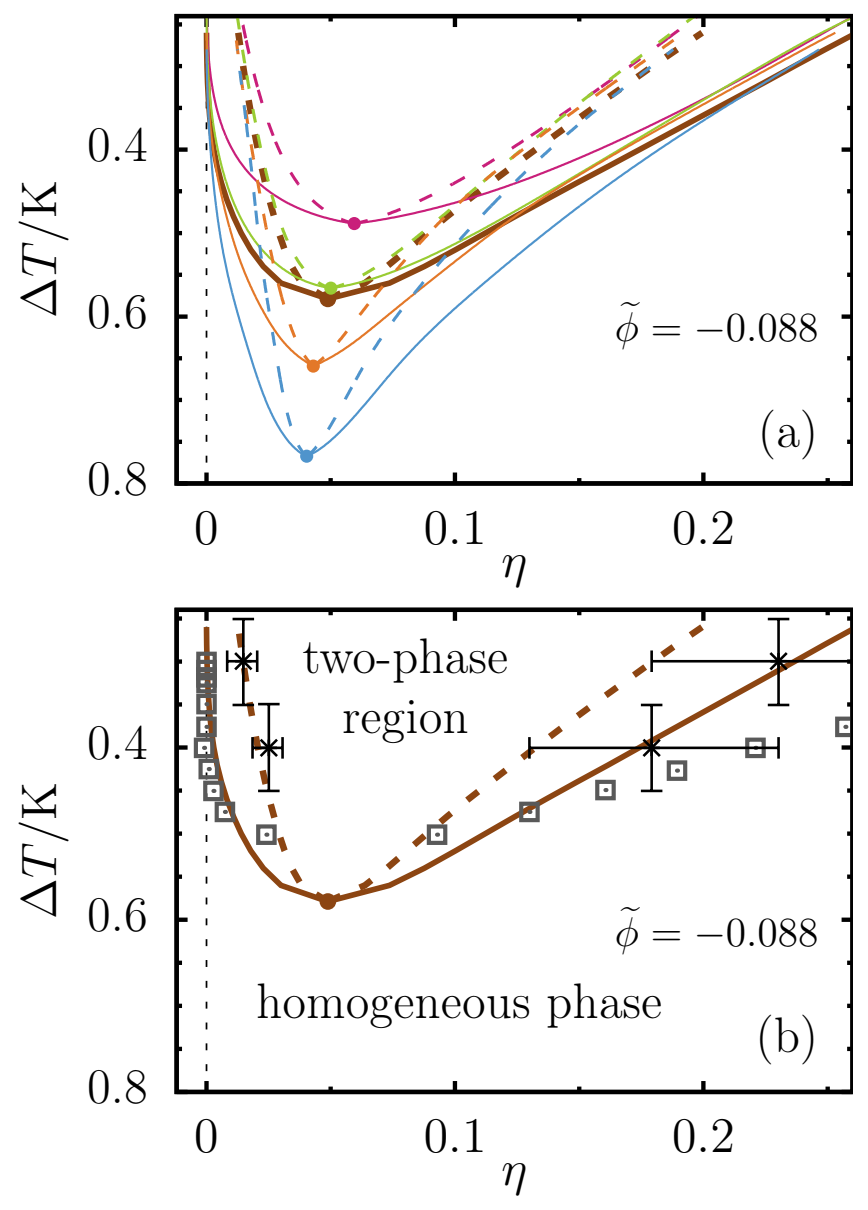

FIG. 10. Segregation phase diagrams obtained from theory (RPA), experiment, and simulations (MC) (Fig. 6 in Ref. [70]). (a) The phase diagram obtained within RPA using the four available background potentials $U_{b c k}$ extracted from effective potentials inferred from experimental data [70] at $\Delta T / \mathrm{K}=0.6$ (magenta), 0.5 (green), 0.4 (orange), and 0.3 (blue), and their mean curve (thick dark red curve). The solid lines show the phase boundaries in terms of the packing fraction $\eta$ of the colloids, whereas the dashed lines correspond to the spinodals, and dots represent critical points. (b) Comparison of the theoretical predictions for the phase boundaries (based on the mean $\left.U_{b c k}\right)$ with MC simulation data $(\odot)$ and experimental data $(\times$, with error bars) from Ref. [72]. On the temperature axis $\Delta T=T_{c}^{(s)}-T$ increases from top to bottom in order to mimic the visual impression of a lower critical point $T_{c}^{(s)}$ (of the solvent) as observed experimentally. $\widetilde{\phi}=$ $\left(\omega_{3 M P, c}-\omega_{3 M P}\right) / \mathcal{B}=-0.088$, where $\mathcal{B}$ is the non-universal amplitude of the bulk coexistence curve (see the main text). 

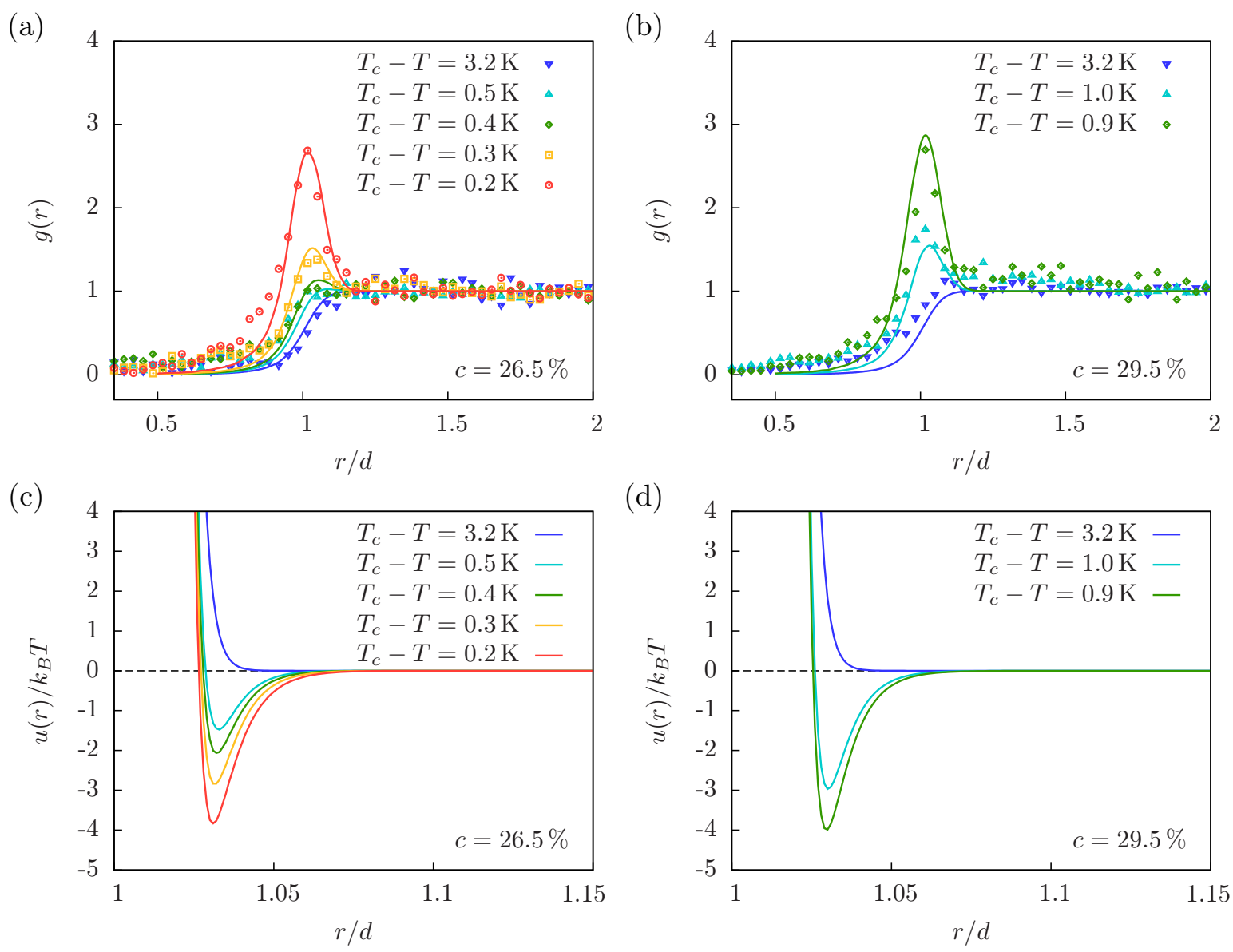

FIG. 11. (a) and (b): Radial distribution function $g(r)$ of the PNIPAM particles suspended in the $3 \mathrm{MP}$ - heavy water liquid mixture for the off-critical mass fractions $\omega_{3 M P}$ (in the figures denoted as $c$ and given in unites of weight percentage wt $\left.\% 3 \mathrm{MP}=100 \times \omega_{3 M P}\right) c=26.5 \%\left(\right.$ with $\left.\Delta T_{\text {off }}=0.7 \mathrm{~K}\right)$ and $c=29.5 \%$ (with $\Delta T_{\text {off }}=0.18 \mathrm{~K}$ ), where the effective temperature offset $\Delta T_{o f f}$ defined via $\left(T_{c}-T+\Delta T_{o f f}\right) / T_{c} \equiv t+t_{o f f}$ is a fit parameter. The experimental data (symbols), obtained in Ref. [86] by using confocal microscopy, are compared with the theoretical predictions (solid lines) based on the model given by Eq. (19) with the Derjaguin approximation for the CCP. (c) and (d): Theoretically predicted pair potentials $u(r)$ for the same compositions (Fig. 7 in Ref. [86]). 
(a)

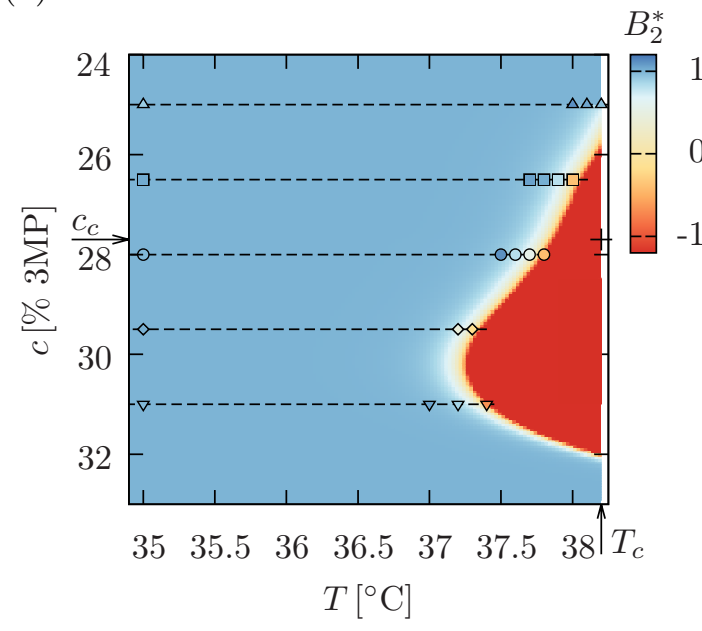

(b)

(c)

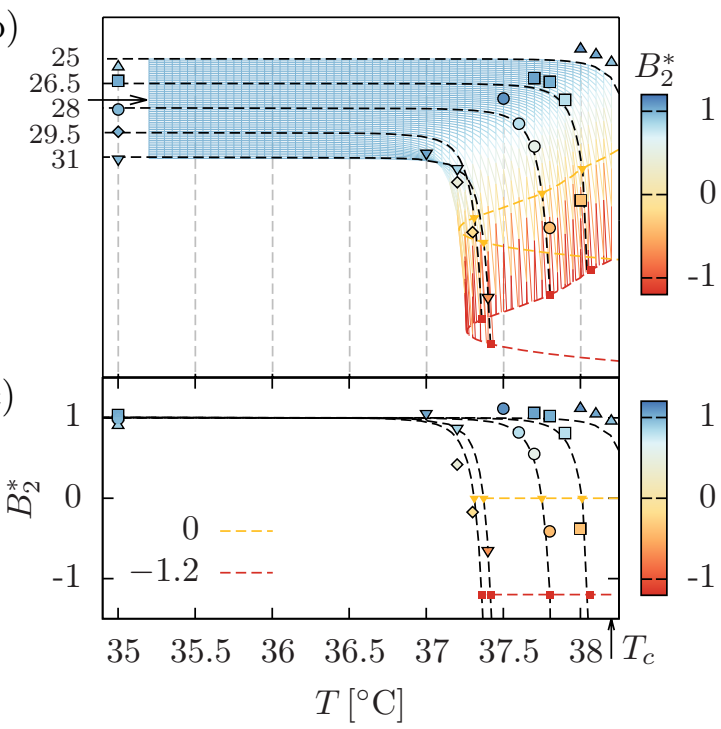

FIG. 12. Reduced second virial coefficient $B_{2}^{*}=B_{2} / B_{2}^{(H S)}$ as function of $T$ and $c$ for the same system as in Fig. 11 (see Fig. 9 in Ref. [86]). The color of the shading provides the theoretically predicted values of $B_{2}^{*}(T, c)$, while the colored symbols provide the experimental value of $B_{2}^{*}(T, c)$ as obtained by numerically integrating the measured radial distribution function $g(r)$ (Eq. (19)). The weak color contrast between the colors of the symbol and the corresponding underlying shading indicates agreement between the experimental and theoretical data. The values $B_{2}^{*} \approx 1$ depicted in blue indicate a significant repulsion, while the values $B_{2}^{*} \approx-1$ depicted in red indicate strong attraction. Yellow marks the crossover. The critical temperature $T_{c}^{(s)}$ (denoted as $T_{c}$ ) and the critical mass fraction $c_{c}$ are indicated by arrows and the critical point is indicated by + . In (a), via the color, $B_{2}^{*}$ is shown in the entire $(T, c)$ plane. The black dashed lines correspond to those five concentrations for which there are experimental data; each dashed line corresponds to a certain symbol type. In (c) the values of $B_{2}^{*}$ are shown as functions of temperature for the five values of $c$ as introduced in (a) via the corresponding symbol type. The horizontal dashed yellow and red lines indicate the isolines for $B_{2}^{*}=0$ and $B_{2}^{*}=-1.2$, marking the crossover from repulsion to attraction and the critical value of the sticky spheres model, respectively. The value of $B_{2}^{*}$ can be read off both from the vertical axis and from the color of the symbols. Panel (b) shows the same data but the curves corresponding to distinct values of $c$ are shifted up vertically in order to gain visual clarity. The shifts are chosen such that the shifted values of $B_{2}^{*}$ at $T=35{ }^{\circ} \mathrm{C}$ are separated equally. This generates a perspective view of the $B_{2}^{*}$ values above the $(T, c)$ plane, revealing a folded curtain-like shape of the surface $B_{2}^{*}(T, c)$. The color shading is discretized due to the shifts of the curves. The yellow and red dashed lines emerge from the corresponding ones in (c) by the shifts. The vertical axis in (b) is $B_{2}^{*}$, but shifted. In the upper left corner of (b), the five dashed lines are labeled both by the symbol types and the corresponding numerical values of the concentration. 


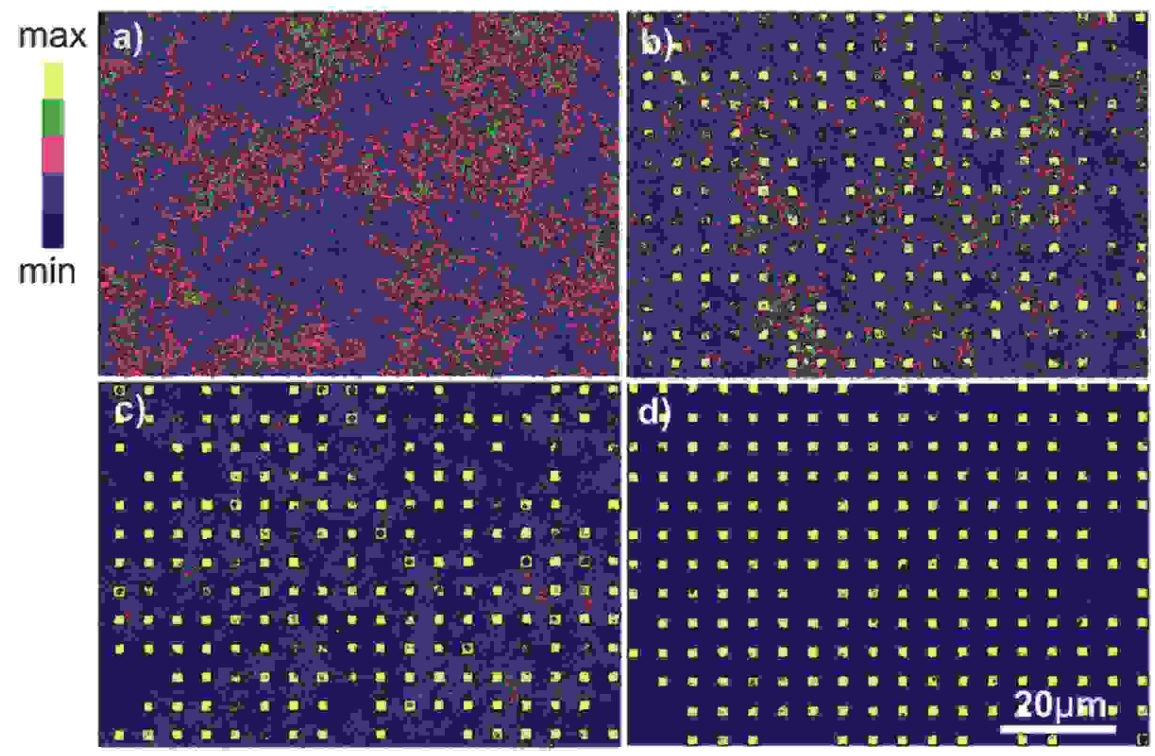

FIG. 13. Mean particle density distribution (represented by different colors ranging from black to yellow for minimal to maximal density, respectively) of a dilute colloidal suspension of spherical particles with $R=2.4 \mu \mathrm{m}$ dissolved in a critical WL mixture in the presence of a chemically patterned substrate (Fig. 1 in Ref. [190]). Particle positions were determined by digital video microscopy with a spatial resolution of ca. $50 \mathrm{~nm} . T_{c}^{(s)}-T=0.72 \mathrm{~K}(\mathrm{a}), 0.25 \mathrm{~K}(\mathrm{~b}), 0.23 \mathrm{~K}(\mathrm{c})$, and $0.14 \mathrm{~K}(\mathrm{~d})$. 


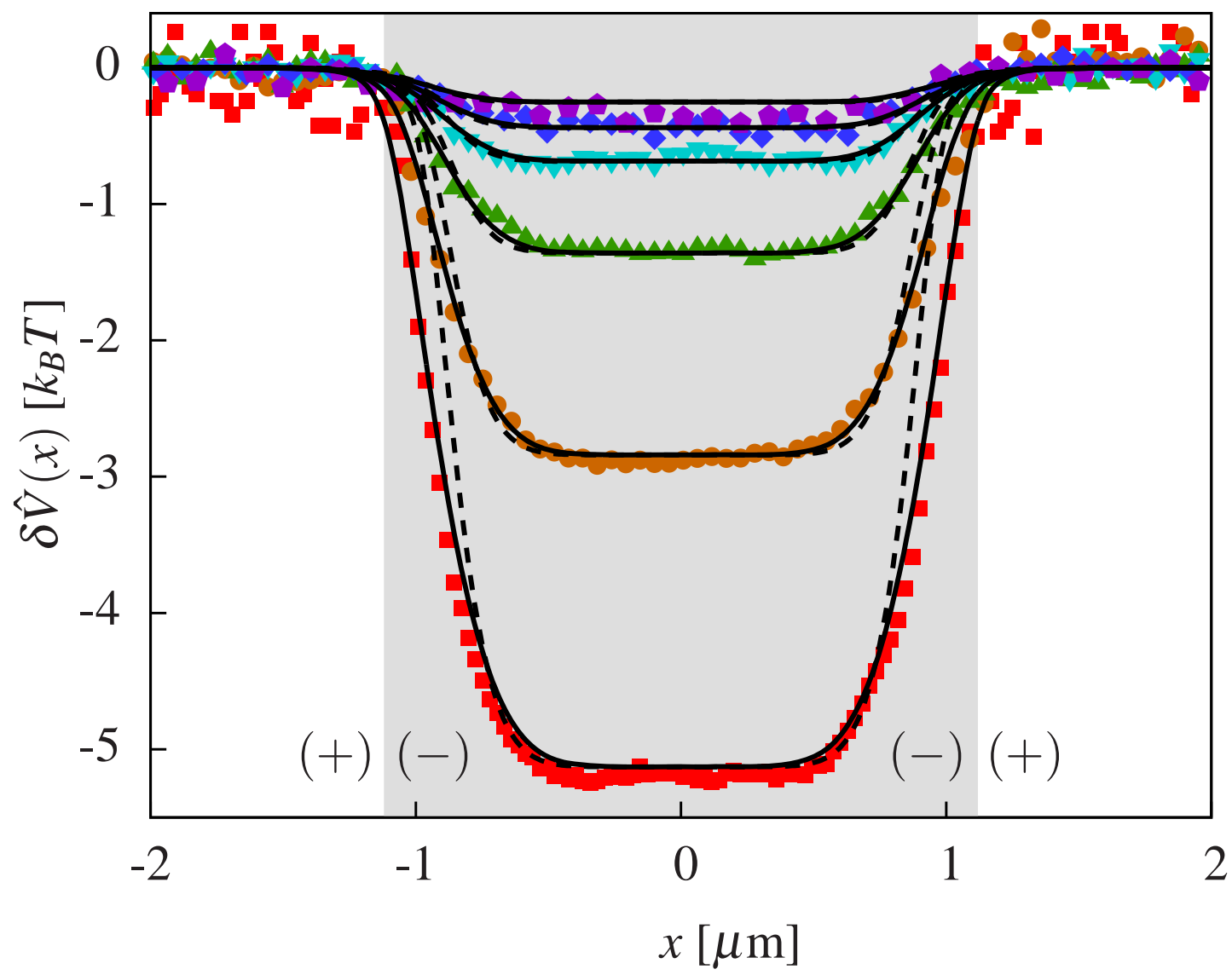

FIG. 14. Total effective potential $\delta \hat{V}(x)=\hat{V}(x)-\hat{V}(x=P / 2)$ of the forces acting on a hydrophilic polystyrene spheres of radius $R=1.2 \mu \mathrm{m}$ above a chemically striped pattern of periodicity $P$ with alternating $(-)$ and $(+)$ boundary conditions and immersed in a water - lutidine mixture at its critical concentration, as function of its lateral position $x$ for various temperatures $T_{c}^{(s)}-\Delta T$ below the experimental value $T_{c}^{(s)}$ of the lower critical temperature of the solvent (Fig. 5 in Ref. [191]). The potential is given by $\hat{V}(x)=-k_{B} T \ln (\hat{\rho}(x))$, where $\hat{\rho}(x)$ is the effective number density of the colloids at $x$, obtained by projecting the actual number density onto the $x$ axis. The width of $(-)$ and $(+)$ stripes is $2.25 \mu \mathrm{m}$. Symbols indicate experimental data, whereas the lines are the corresponding theoretical predictions for sharp (dashed lines) and fuzzy (solid lines) chemical steps. From top to bottom the measured temperature deviations $\Delta T$ are 0.175 (0.165), 0.16 (0.152), 0.145 (0.143), $0.13,0.115,0.10 \mathrm{~K}$. If indicated, the values in paranthesis are corrected values of temperature (but compatible within the experimental inaccuracy) which have been used for evaluating the theoretical predictions. 
a)

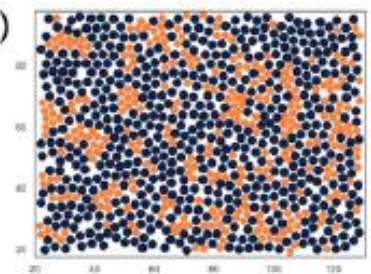

b)

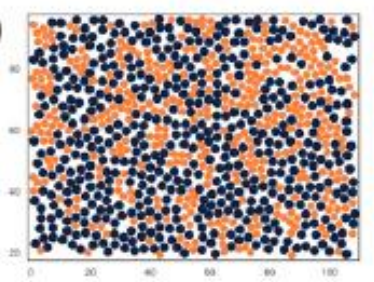

c)

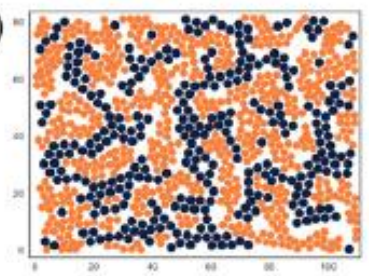

FIG. 15. Snapshots of the twodimensional configurations of binary colloidal systems (black and orange discs, Fig. 1 in Ref. [73]) for three different compositions, expressed in terms of the concentration $x_{a}=\rho_{a} /\left(\rho_{a}+\rho_{b}\right)$, where $\rho_{a}$ and $\rho_{b}$ are (areal) number densities of the particles of type $a$ and $b$, respectively: (a) $x_{a}=0.28$, (b) $x_{a}=0.32$, and (c) $x_{a}=0.54$, with $a=$ orange, exhibiting distinct structures formed after 1 hour at a temperature deviation $\Delta T=T_{c}^{(s)}-T=0.01 \mathrm{~K}$ from the lower critical point of the solvent. The horizontal scales range from 20 to 120 in (a) and from 0 to 100 in (b) and (c), whereas the vertical scales range from 20 to 80 in (a) and (b) and from 0 to 80 in (c); the authors have not provided the units. 


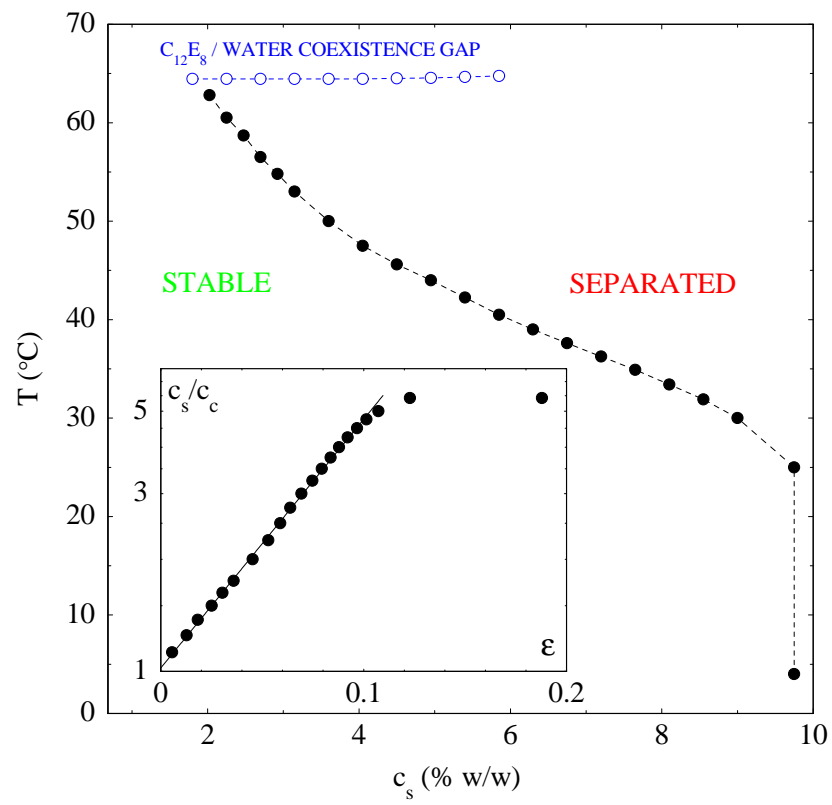

FIG. 16. Experimental phase diagram of aqueous suspensions of MFA latex particles in the presence of a nonionic surfactant of mass fraction $c_{s}=m_{s} / m_{t o t}$ (in units of mass percentage, sometimes called weight percentage, $\% \mathrm{w} / \mathrm{w}=100 \times c_{s}$ ), where $m_{s}$ is the mass of all surfactant particles and $m_{\text {tot }}$ is the total mass of the sample (Fig. 8 in Ref. [78]). For state points of the surfactant - water mixtures to the left of the line of dots (denoted as "stable") the dissolved colloidal particles with a volume fraction $V_{c} / V_{t o t}=0.03$ form a homogeneous colloidal phase whereas for state points to the right of the line of dots (denoted as "separated") the colloids phase separate into a colloidal gas and a colloidal liquid. ( $V_{c}$ is the volume taken by colloidal particles and $V_{t o t}$ is the total volume of the sample). At a given temperature, the full black dots represent the minimum amount $c_{s}^{(m)}$ of surfactant required to induce colloidal gas-liquid phase separation at a given temperature. The rather shallow coexistence curve of the surfactant-water mixture with a lower critical point (located around $1.8 \%$ of $c_{s}$ - not marked in the plot) is shown by open blue dots. The data correspond to $250 \mathrm{mM}$ added $\mathrm{NaCl}$ salt. The inset shows (in our present notation) $c_{s}^{(m)}$ in units of its critical value $c_{s}^{(c)}$ (i.e., $c_{s}$ at the critical point) as a function of reduced temperature $t=\left(T_{c}^{(s)}-T\right) / T_{c}^{(s)}$ (note that - as in Ref. [78] - in this figure $c_{s}^{(m)} / c_{s}^{(c)}$ and $t$ are denoted by $c_{s} / c_{c}$ and $\varepsilon$, respectively.) 


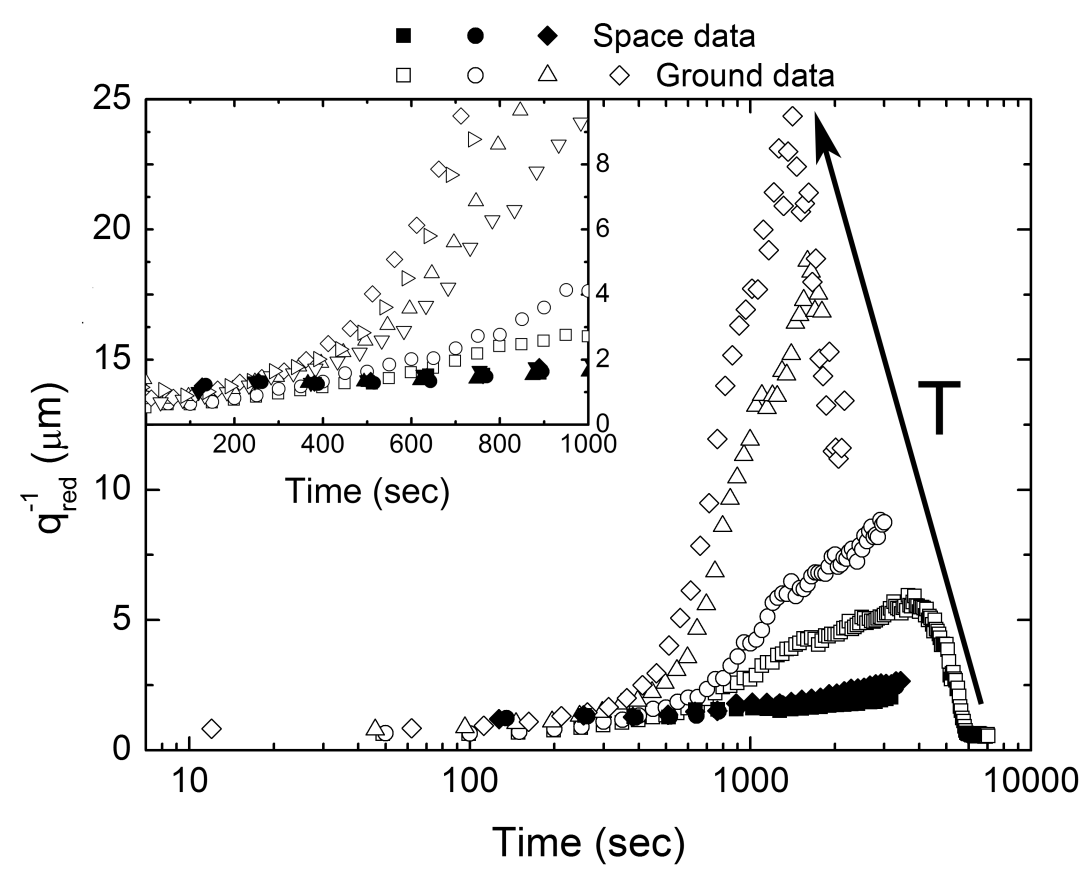

FIG. 17. The evolution of the characteristic size scale of the aggregating colloidal suspension studied in Ref. [194] (see Fig. 3 therein) as given by the quantity $q_{r e d}^{-1}(\mathrm{t})$, which renders a reduced description of the light scattering intensity $I(q, \mathrm{t})$ of the growing aggregates in terms of a scaling function $F\left(q / q_{\text {red }}(\mathrm{t})\right)$ of a single variable (see the main text). Results both from microgravity (full symbols) and from ground experiments (open symbols) are shown. (The sample contains $1.5 \mathrm{mmol} /$ liter $\mathrm{NaCl}$.) The curves correspond to various temperatures $T$ ramped up beyond the aggregation temperature $T_{a g g}$, up to $T_{a g g}+0.4 \mathrm{C}$ (from bottom to top as indicated by the arrow; the caption to Fig. 3 in Ref. [194] does not provide the temperature values corresponding to the various symbols). The temperature $T_{a g g}$ at which the aggregation starts is identified (somewhat loosely) as the onset of the rapid increase of the normalized variance of $I(q)$ (see the main text). The sudden drop of $q_{r e d}^{-1}$ at late times is due to the massive sedimentation of the aggregates so that the suspension becomes poor in large aggregates and rich in small ones which shifts down $q_{\text {red }}^{-1}$. The inset provides an enlarged view of the data concerning the onset of the aggregation process, at which the characteristic length scale $q_{r e d}^{-1}$ starts to increase. 


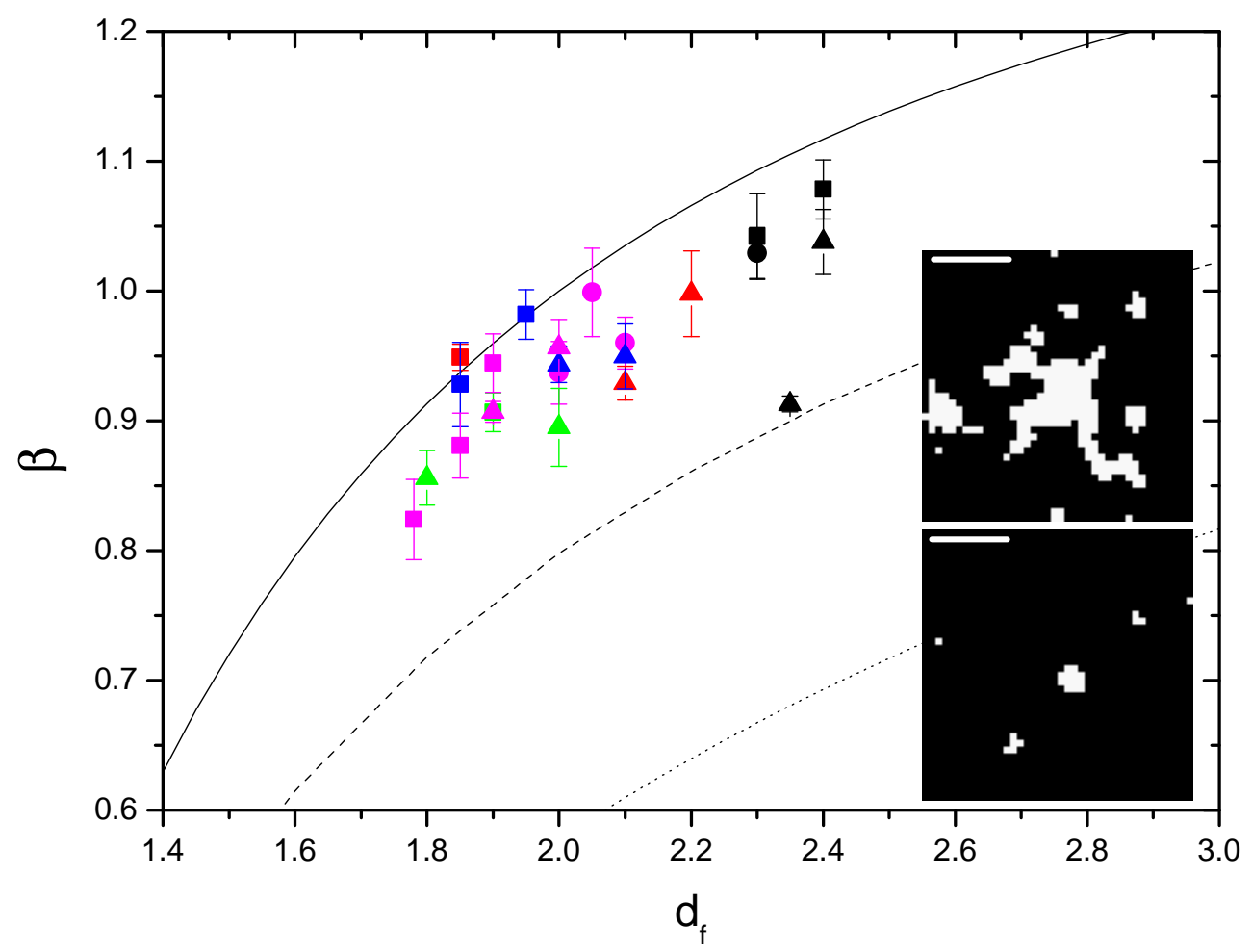

FIG. 18. Ratio $\beta=R_{h} / R_{g}$ of the hydrodynamic to the gyration radius of aggregates as a function of their fractal dimension $d_{f}$ for various temperature deviations $\Delta T$ above the aggregation temperature $T_{a g g}$ (Fig. 4 in Ref. [195]) characterized by the colors of the symbols: $\Delta T=0$ (black), $0.1 \mathrm{~K}$ (red), $0.2 \mathrm{~K}$ (blue), $0.3 \mathrm{~K}$ (green), and $0.4 \mathrm{~K}$ (violet). $T_{a g g}$ is determined as in Fig. 18, i.e., from the behavior of the normalized variance of the scattered intensity $I(q)$. The types of symbols indicate the salt concentrations of $0.31 \mathrm{mmol} /$ liter (squares), 1.5 (circles), and 2.7 (triangles), corresponding to the Debye screening length $\kappa^{-1}$ (denoted as $\lambda_{D}$ in Ref. [195]) of 14, 6.4, and $4.8 \mathrm{~nm}$, respectively. Concerning the values of $\beta$, from top to bottom the lines indicate the dependence on $d_{f}$ as expected for steplike, Gaussian, and exponentially decaying cut functions of the density distribution of the aggregates, respectively (see the main text). The insets show holographic reconstructions of the the real-space shape of the aggregates (white regions) grown at $T=T_{a g g}+0.4 \mathrm{~K}$ (closest to $T_{c}^{(s)}$ and hence strongest attraction between the colloids, top) and $T=T_{a g g}$ (furthest from $T_{c}^{(s)}$ and hence weakest attraction between the colloids, bottom). The length of the scale bar is $25 \mu \mathrm{m}$. 

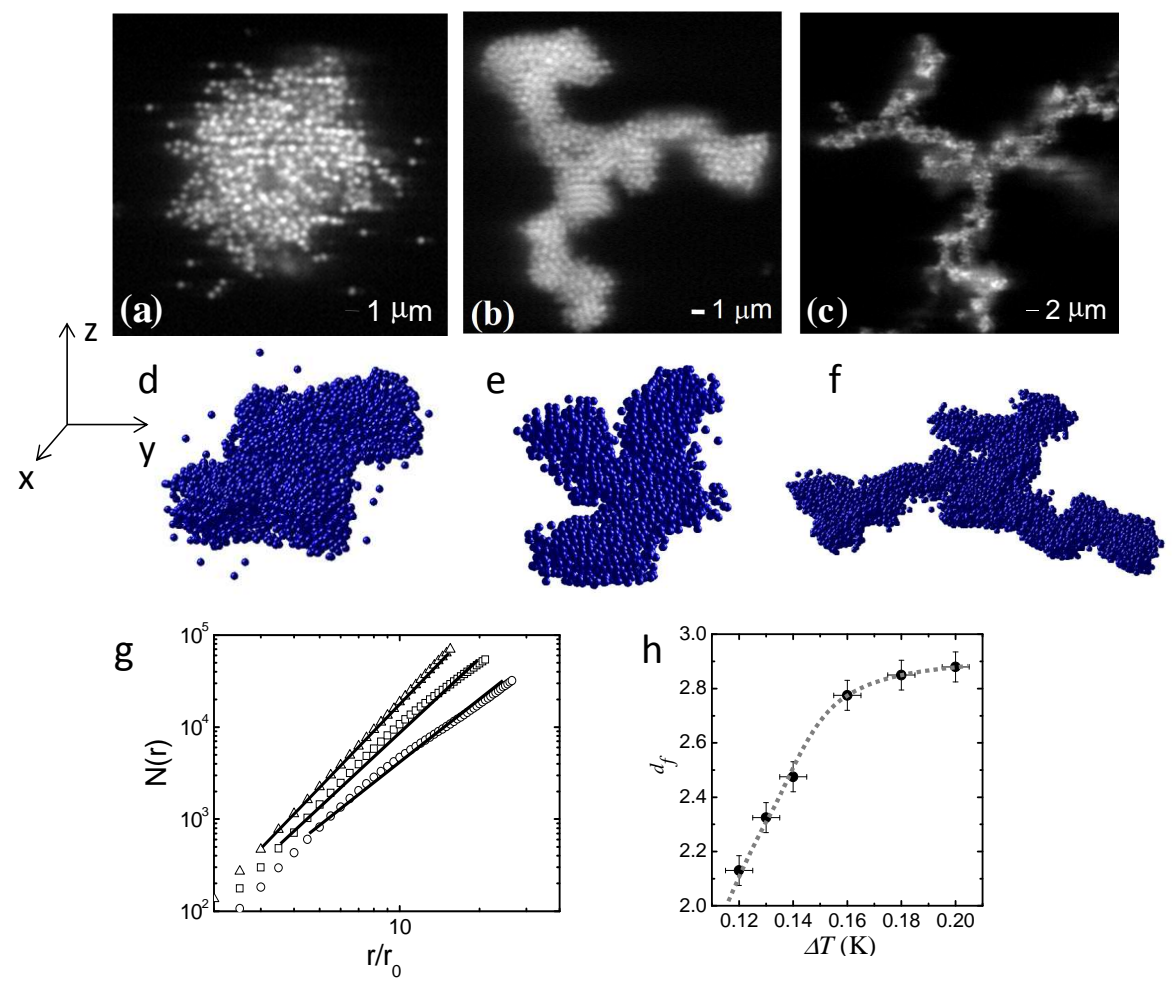

FIG. 19. Tuning the morphology of colloidal aggregates by critical Casimir forces (Fig. 1 in Ref. [85]). Confocal microscopy images and the threedimensional reconstructions of the aggregates formed upon temperature quenches to $\Delta T=T_{c}^{(s)}-T=0.2 \mathrm{~K}(\mathrm{a}, \mathrm{d}), 0.14 \mathrm{~K}(\mathrm{~b}, \mathrm{e})$, and $0.12 \mathrm{~K}$ $(\mathrm{c}, \mathrm{f})$ below the lower critical phase separation temperature $T_{c}^{(s)}$ of the binary solvent. (g) Scaling of the number of particles $\mathcal{N}(r)$ within a sphere of radius $r$ from the center of the cluster at $\Delta T=0.2 \mathrm{~K}$ (triangles), $\Delta T=0.14 \mathrm{~K}$ (squares), and $\Delta T=0.12 \mathrm{~K}$ (circles); $r_{0}$ is the radius of the particles. $(\mathrm{h})$ Fractal dimension as a function of $\Delta T$ determined from $\mathcal{N}(r) \sim r^{d_{f}} f_{\text {cut }}(r)$ and thus from the slopes in $(\mathrm{g})$. The dashed line is a guide to the eye. Upon increasing $\Delta T$ a continuous increase of the fractal dimension to the space-filling limit $d_{f}=3$ is observed (see the main text). 